\title{
A STUDY OF THE ELECTROMAGNETIC FIELDS DISTRIBUTION INSIDE BUILDINGS WITH APERTURES EXCITED BY AN EXTERNAL SOURCE
}

Mark T. Ma

M. G. Arthur

National Bureau of Standards U.S. Department of Commerce Boulder, Colorado 80303

February 1982

$-Q C$
100
.456
$82-1659$
1932
$C .2$





\section{A STUDY OF THE ELECTROMAGNETIC FIELDS DISTRIBUTION INSIDE BUILDINGS WITH APERTURES EXCITED BY AN EXTERNAL SOURCE}

Mark T. Ma

M. G. Arthur

Electromagnetic Fields Division National Engineering Laboratory National Bureau of Standards U.S. Department of Commerce Boulder, Colorado 80303

February 1982

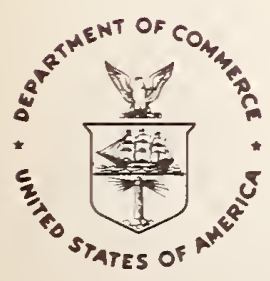

U.S. DEPARTMENT OF COMMERCE, Malcolm Baldrige. Secretary 

CONTENTS

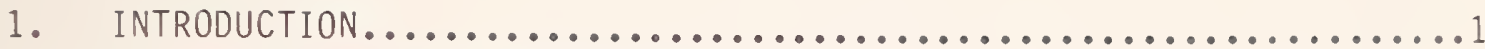

2. A THREE-DIMENSIONAL CONDUCTING

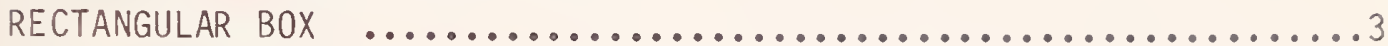

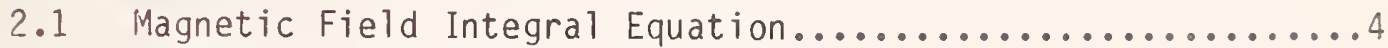

2.2 Generalized Impedance Approach.......................

3. LOSSY BUILDINGS WITH SMALL APERTURES ......................

4. LARGE BUILDINGS AND APERTURES WITH LOSSY

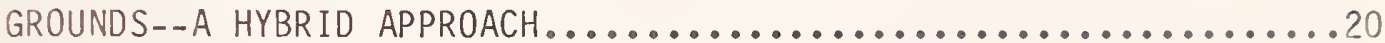

5. MEASUREMENT REQUIREMENTS AND PROCEDURES $\ldots \ldots \ldots \ldots \ldots \ldots \ldots \ldots \ldots \ldots \ldots$

6. COMPUTING REQUIREMENTS AND PROCEDURES $\ldots \ldots \ldots \ldots \ldots \ldots \ldots \ldots \ldots \ldots \ldots$

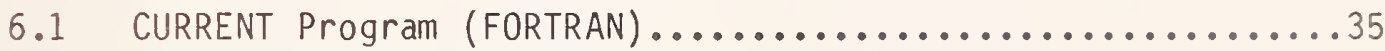

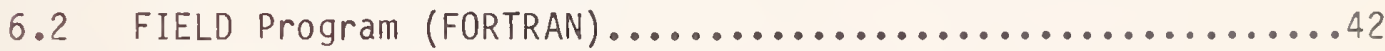

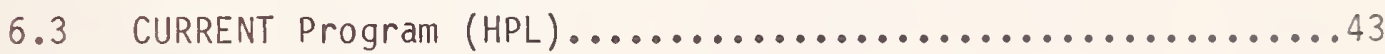

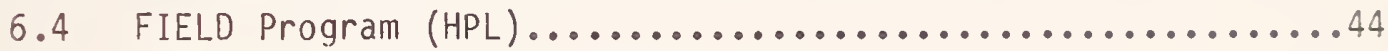

6.5 HPL File Structure on Magnetic Tape...................45

6.6 Print Options for HPL Programs.....................45

7. NUMERICAL RESULTS FOR A PRACTICAL BUILDING...............47

8. CONCLUDING REMARKS..................................66

9. ACKNOWLEDGMENTS .................................... 66

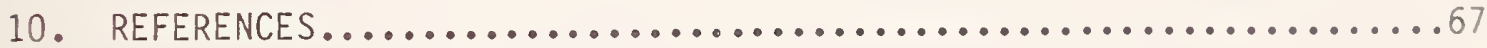

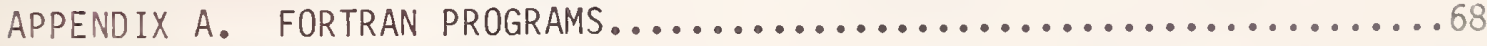

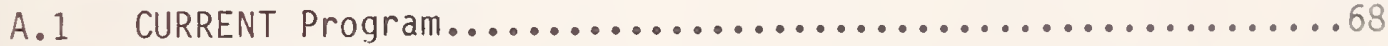

A.2 FIELD Program...................................

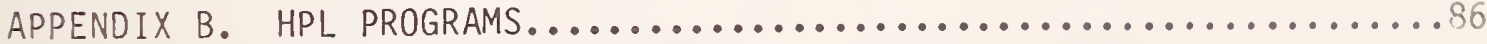

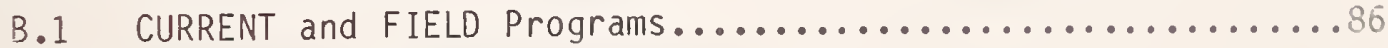

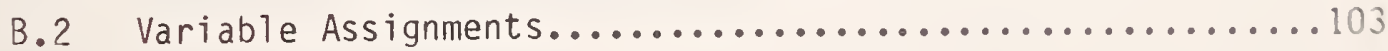

B.3 Instructions for Running HPL Programs...................

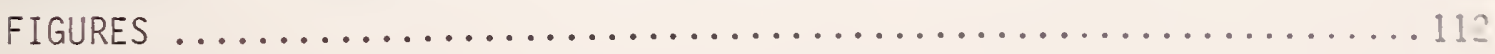



A STUDY OF THE ELECTROMAGNETIC FIELDS DISTRIBUTION

INSIDE BUILDINGS WITH APERTURES EXCITED BY AN EXTERNAL SOURCE

\author{
M. T. Ma and M. G. Arthur
}

Two special cases of the penetration of electromagnetic fields into a cavity, building or box are formulated and analyzed. One is to consider the case of a lossy cavity with small apertures in free space, based on an application of the equivalence principle and the use of a generalized network formulation. It is found that the field strength at the aperture center is approximately inversely proportional to the square-root of the conductivity of the cavity walls and that high field levels can exist inside the cavity under certain physical conditions. The second case is to treat the problem of large buildings with large apertures on a practical lossy ground by a combination of theoretical approach and limited measurement data. Field levels inside the building for this latter case depend on the transmitter power, the transmitter-to-building distance, the ground conductivity, and the measurement antenna height relative to that of the transmitter.

Keywords: aperture; cavity; equivalence principle; field distribution; slot.

\title{
1. INTRODUCTION
}

The capability of electromagnetic waves to penetrate into a building or a box which houses important, sensitive devices and equipment has always been of concern to industries and military installations. Generally speaking, it is essential to know the level of the field strength and its component amplitudes and phases inside the building before an assessment of hazardous effects on equipment performance and human health can be made. Genuine efforts have been made to make on-site spot measurements inside an empty building for a known transmitter at a specified distance away from the building. It is still impossible from these spot measurements to make any meaningful predictions of the highest field level and its polarization inside the building, which are the ultimate goals of this study. Thus, to achieve these goals, systematic measurements of component field amplitudes and phases along three orthogonal directions at an interval distance of no more than one quarter wavelength would be required. These systematic measurements are, of course, very timeconsuming. For a transmitter operated at $150 \mathrm{MHz} \quad(\lambda=2 \mathrm{~m})$, which is typical for a land mobile radio, and a practical storage building of the size of $7 \times \times 141$ $x 10 \lambda$, a huge number of measurement data in the order of one third of a million data points have to be taken and analyzed. Furthermore. these 
measurement data are only applicable to the specific building and depend on construction material, building size and shape, the aperture (doors or windows) size and shape, the ground and surrounding conditions, the operating frequency of the transmitter, and the transmitter-to-building distance under study.

To circumvent this measurement impracticality, a theoretical study of the generalized three-dimensional problem was deemed desirable. However, in view of the complexity of the problem involved and the enormous demand on the computing facility for a practical size of building, only special cases for a CW excitation subject to certain physical constraints are considered in this report.

In Section 2, the problem of a three-dimensional closed conducting rectangular box in free space illuminated by a linearly polarized, normally incident, plane wave is considered with two different approaches. One is by the magnetic field integral equation with appropriate boundary conditions [14], and the other is through the concept of a generalized impedance matrix. Since there is no opening on the box surface in this case, no field can penetrate into the box. Numerical results for the induced surface current densities, on a limited basis are given therein, based on which the field scattered by the box may be computed.

The problem is then extended to the case of lossy rectangular buildings or boxes with small slot apertures in free space, which is presented in Section 3. Field strengths inside the box are shown to be dependent on the aperture and box sizes relative to the operating wavelength and the material of the box walls.

It is found that the field strength at the aperture center is approximately inversely proportional to the square-root of the conductivity of the wall material and that high levels of the field strength can exist inside the box at natural resonances and under a set of special physical conditions.

In Section 4, large buildings and large apertures located on a practical lossy ground are analyzed with a combination of theoretical consideration and limited set of measurement data. Requirements on the measurement equipment and computing facilities for this case are outlined in Section 5 with the measurement and computing procedures presented in Section 6. Numerical results of the approximate induced current densities and the approximate field distribution inside the building for a known transmitter frequency, power, and the transmitter-to-building distance are given in Section 7 . Physical 
interpretations of these numerical results and the limiting conditions under which the results have meanings are discussed in the concluding section. A relevant FORTRAN program used to obtain the numerical results is listed in Appendix A. A simplified version using the HPL* suitable to Model HP 9825* capable of making a quick on-site check with a much reduced matrix size is presented in Appendix B.

\section{A THREE-DIMENSIONAL CONDUCTING RECTANGULAR BOX}

The geometry depicted in figure 1 may be used to represent a theoretical model for the problem of the penetration of electromagnetic waves into a rectangular cavity (building or box). The source located outside the cavity may be a CW or pulsed signal of arbitrary polarization and frequency, incident on the cavity with an arbitrary angle. The cavity walls are generally made of non-perfectly conducting material, and the cavity itself is situated on a homogeneous flat lossy earth represented by the ground constants of permeability $(\mu)$, permittivity $(\varepsilon)$ and conductivity $(\sigma)$.

The objective of formulating and analyzing this problem is to determine the spatial field distribution and locations of the highest levels of field strength inside the cavity as a function of many parameters such as the sizes of the cavity and the aperture, the ground constants, etc. Obviously, this type of problem is too complicated to be treated rigorously using Maxwell's equations with appropriate boundary conditions. To simplify the problem, a three-dimensional perfectly conducting rectangular box without any apertures located in free space, illuminated by an external source, as shown in figure 2 may be considered as a first approximate model to determine the induced surface current densities, from which the scattered field outside the box may then be calculated. The numerical results so obtained can be compared with the existing measurement data on radar cross-sections to assess the validity of the formulation and the accuracy of the numerical computations.

*"Certain commercial equipment, instruments, or materials are identified in this paper in order to adequately specify the experimental procedure. Such identification does not imply recommendation or endorsement by the National Bureau of Standards, nor does it imply that the materials or equipment identified are necessarily the best available for the purpose." 


\subsection{Magnetic Field Integral Equation}

Two approaches have been available for deriving the necessary integral equations to determine the current densities induced on the surfaces of the box in figure 2. The first is to formulate the electromagnetic boundary-value problem in terms of the magnetic field integral equation, which takes the following form [5]:

$$
\frac{1}{2} \bar{j}(\bar{r})=\hat{n} \times \bar{H}_{i}(\bar{r})+\frac{1}{4 \pi} \hat{n} \times f_{s^{\prime}} \bar{j}\left(\bar{r}^{-}\right) \times \nabla^{-} G\left(\bar{r}, \bar{r}^{-}\right) d s^{\prime}
$$

where $\bar{H}_{j}(\bar{r})$ is the known incident magnetic field, $\bar{j}(\bar{r})$ is the unknown tangential current density induced on the six box surfaces,

$$
\begin{gathered}
G\left(\bar{r}, \bar{r}^{\prime}\right)=\exp (-j k R) / R \\
R=\left|\bar{r}-\bar{r}^{-}\right|=\left[\left(x-x^{\prime}\right)^{2}+\left(y-y^{\prime}\right)^{2}+\left(z-z^{\prime}\right)^{2}\right]^{1 / 2} \\
r=\hat{x} x+\hat{y} y+\hat{z} z \\
\bar{r}^{-}=\hat{x} x^{\prime}+\hat{y} y^{-}+\hat{z} z^{\prime}
\end{gathered}
$$

$x, x^{\prime}, y, y^{\prime}, z, z^{\prime}=$ coordinates on the box surfaces

$\hat{x}, \hat{y}$ and $\hat{z}=$ unit vectors in $x, y$, and $z$ directions

$$
\nabla^{\prime}=\hat{x} \frac{\partial}{\partial x^{\prime}}+\hat{y} \frac{\partial}{\partial y^{\prime}}+\hat{z} \frac{\partial}{\partial z^{\prime}}
$$

$$
\begin{aligned}
\hat{n}= & \text { a unit vector in the outward normal direction to each box } \\
& \text { surface } \\
S= & \text { the area of each of the six surfaces, }
\end{aligned}
$$

and the horizontal bar across the integral sign in (1) denotes that only the principal value will be taken. 
Since there are two components of $\bar{j}(\bar{r})$ on each surface of the bux, equation (1) results in 12 coupled scalar integral equations for the component current densities. As an example, in reference to figure 2, we have

$$
\bar{H}_{i}(\bar{r})=\hat{x} H_{i x}(\bar{r})+\hat{z} H_{i z}(\bar{r}),
$$

for the wave propagating in the $y$-direction, normal to Face I (right). Tnus, for faces I and II (right and left),

$$
\bar{j}\left(\bar{r}^{-}\right)=\hat{x} J_{x}\left(\bar{r}^{-}\right)+\hat{z} J_{z}\left(\bar{r}^{\prime}\right),
$$

for faces III and IV (top and bottom),

$$
\vec{j}\left(\vec{r}^{-}\right)=\hat{x} J_{x}\left(\bar{r}^{-}\right)+\hat{y} J_{y}\left(\bar{r}^{-}\right),
$$

for faces $V$ and VI, (front and back)

$$
\bar{j}\left(\bar{r}^{-}\right)=\hat{y} J_{y}\left(\bar{r}^{-}\right)+\hat{z} J_{z}\left(\bar{r}^{-}\right),
$$

and

$$
\begin{aligned}
\nabla^{\prime} G\left(\vec{r}, \vec{r}^{-}\right) & =\left(\hat{x} \frac{\partial}{\partial x^{\prime}}+\hat{y} \frac{\partial}{\partial y^{\prime}}+\hat{z} \frac{\partial}{\partial z^{\prime}}\right) \exp (-j k R) / R \\
& =\hat{x} \psi_{x}+\hat{y} \psi_{y}+\hat{z} \psi_{z}
\end{aligned}
$$

where

and

$$
\begin{aligned}
& \psi_{x}=\left(j k+\frac{1}{R}\right)\left(x-x^{\prime}\right) \exp (-j k R) / R^{2}, \\
& \psi_{y}=\left(j k+\frac{1}{R}\right)\left(y-y^{\prime}\right) \exp (-j k R) / R^{2},
\end{aligned}
$$

$$
\psi_{z}=\left(j k+\frac{1}{R}\right)\left(z-z^{\prime}\right) \exp (-j k R) / R^{2} .
$$


The integrand of (1) then becomes

for Faces I and II,

$$
\hat{x}\left(-J_{z} \psi_{y}\right)+\hat{y}\left(\psi_{x} J_{z}-J_{x} \psi_{z}\right)+\hat{z}\left(J_{x} \psi_{y}\right),
$$

for Faces III and IV,

$$
\hat{x}\left(J_{y} \psi_{z}\right)+\hat{y}\left(-J_{x} \psi_{z}\right)+\hat{z}\left(J_{x} \psi_{y}-\psi_{x} J_{y}\right),
$$

and for Faces $V$ and $V I$,

$$
\hat{x}\left(J_{y} \psi_{z}-J_{z} \psi_{y}\right)+\hat{y}\left(J_{z} \psi_{x}\right)+\hat{z}\left(-\psi_{x} J_{y}\right) .
$$

After taking the principal value of (1), we finally have, for Face I where $\hat{n}=-\hat{y}$,

$$
\frac{1}{2} J_{x}(\bar{r})=-\bar{H}_{i z}(\bar{r})-\frac{1}{4 \pi} \int_{\text {I I , I I I , IV }} J_{x}\left(r^{\prime}\right) \psi_{y} d s^{\prime}+\frac{1}{4 \pi} \int_{\text {I I I , IV , V , V I }} J_{y}\left(r^{\prime}\right) \psi_{x} d s^{\prime}
$$

and

$\frac{1}{2} J_{z}(\bar{r})=H_{i x}(r)-\frac{1}{4 \pi} \int_{I I, V, V I} J_{z}\left(r^{\prime}\right) \psi_{y} d s^{\prime}+\frac{1}{4 \pi} \int_{\text {II I , IV V V V VI }} J_{y}\left(r^{\prime}\right) \psi_{z} d s^{\prime}$.

Equations similar to (18) and (19) can be obtained for Faces II through VI, resulting in exactly 12 coupled scalar integral equations. If the method of moments [6] is used to solve for this system of equations by subdividing each surface into $n \times n$ patches, we will end up $12 n^{2} \times 12 n^{2}$ simultaneous linear algebraic equations. When the value of $n$ is sufficiently large to insure meaningful results, the inversion of a matrix size involved may be prohibitive even with the availability of a large computer capacity. The results of $\left|J_{x}\right|$ and $\left|J_{z}\right|$ on Face I for a $(0.5 \lambda)^{3}$ cube $i l l u m i n a t e d$ by a broadside incidence plane wave $\mathrm{H}_{i z}$ with unity strength are presented in figure 3 , which were obtained with $8 \times 8$ patches [5]. Similar results for other box surfaces are also available [5]. Based on the results of surface current densities, the scattered fields outside the cube may then be computed. For larger sizes of cube or arbitrary incidence, the numerical procedures are more involved. 


\subsection{Generalized Impedance Approach}

An alternative approach to solving the problem depicted in figure 2 is by using the concept of the generalized impedance matrix. Basically, the electric field scattered by the box shown in figure 2 can be determined by

$$
\bar{E}_{S}=-j \omega \bar{A}-\nabla \phi,
$$

where $\bar{A}$ and $\phi$ are, respectively, the magnetic vector potential and the electric scalar potential, satisfying the following equations:

$$
\bar{A}=\frac{\mu}{4 \pi} \int_{S} \bar{J}\left(\bar{r}^{-}\right) G\left(\bar{r}, \bar{r}^{-}\right) \mathrm{ds}^{-}
$$

and

$$
\phi=-\frac{1}{j \omega 4 \pi \varepsilon} \int_{S} \nabla^{\prime} \cdot \bar{j}\left(\bar{r}^{-}\right) G\left(\bar{r}, \bar{r}^{\prime}\right) \mathrm{d} s^{\prime}
$$

where $\bar{J}$ and $G$ have been explained or defined in (1) and (2).

On the surface of a perfectly conducting box, the tangential component of the total electric field vanishes, or

$$
\bar{E}_{i, \tan }=-\bar{E}_{s, \tan }
$$

where $\bar{E}_{i, t a n}$ is the tangential component of the electric field at the box urface due to the incident signal, and $\bar{E}_{S, t a n}$ is the tangential component of lat in (20).

Combining (20) and (23), we obtain

$$
\begin{aligned}
\bar{E}_{i, \tan } & =\left[\frac{j \omega \mu}{4 \pi} \int_{S} \bar{j}\left(\bar{r}^{\prime}\right) G\left(\bar{r}, \bar{r}^{-}\right) \mathrm{ds}^{-}\right. \\
& \left.+\frac{1}{4 \pi j \omega \varepsilon} \nabla \int_{S} \bar{j}\left(\bar{r}^{\prime}\right) \cdot \nabla^{-} G\left(\bar{r}, \bar{r}^{-}\right) d s^{-}\right]_{\tan } \\
& =L\left[\bar{j}\left(\bar{r}^{\prime}\right)\right]
\end{aligned}
$$

where the linear operator $L$ represents the necessary double integrals. 
Using the standard method of moments [6], we subdivide the surface $S$ into patches and expand the components of the unknown surface current density function $\bar{j}\left(\bar{r}^{-}\right)$as follows:

$$
J_{j}\left(\bar{r}^{\prime}\right)=j-t h \text { component of } \bar{j}\left(\bar{r}^{-}\right)=\sum_{n=1}^{n} I_{n} f
$$

where $f_{n}$ is a set of chosen basis functions, and $I_{n}$ 's are complex coefficients to be determined.

Substitution of (25) into (24) yields

$$
\left[Z_{m n}\right]\left[I_{n}\right]=\left[v_{m}\right]
$$

where

$$
\begin{aligned}
& {\left[I_{n}\right]=\left(\begin{array}{c}
I_{1} \\
I_{2} \\
\vdots \\
I_{N}
\end{array}\right)}
\end{aligned}
$$

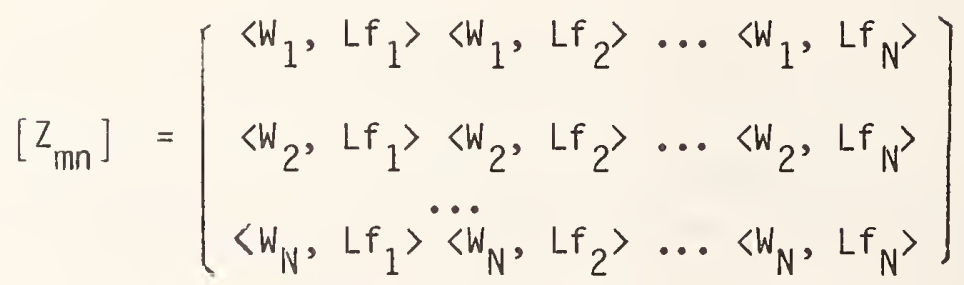

$$
\begin{aligned}
& {\left[v_{m}\right]=\left(\begin{array}{cc}
\left\langle W_{1},\right. & \left.E_{i, j}\right\rangle \\
\left\langle W_{2},\right. & \left.E_{i, j}\right\rangle \\
\left\langle W_{N},\right. & \left.E_{i, j}\right\rangle
\end{array}\right)} \\
& E_{i, j}=\text { the } j \text {-th component of } \bar{E}_{i, \tan } \text { in (24), }
\end{aligned}
$$


$W_{1}, W_{2}, \ldots W_{N}$ constitute a set of testing functions which are tangential to $S$, and $\langle a, b\rangle$ represents an inner product defined as:

$$
\langle a, b\rangle=\int a b d s^{-}
$$

The elements of $\left[Z_{m n}\right]$ in (28) may be called generalized impedances; those of $\left[\mathrm{I}_{n}\right]$ in (27) generalized currents; and those of $\left[\mathrm{V}_{m}\right]$ in (29) generalized voltages representing the sources. When $\left[z_{m}\right]$ is nonsingular which is true in general, its inverse $\left[z_{m n}\right]^{-1}$ exists. Then, we have

$$
\left[I_{n}\right]=\left[Z_{m n}\right]^{-1}\left[V_{m}\right]
$$

which is the set of coefficients required in (25) for obtaining an approximate component of $\bar{j}\left(\bar{r}^{-}\right)$.

Specific numerical results of $\left|\mathrm{J}_{x} / \mathrm{H}_{i z}\right|$ and $\left|\mathrm{J}_{z} / \mathrm{H}_{i z}\right|$ on Face I of figure 2 for $(0.1 \lambda)^{3}$ cube with the same normal incidence are given in figures 4 and 5. From figure 4, it is clear that $\left|J_{x} / H_{i z}\right|$ is again the dominant response for the particular plane-wave incidence being considered.*

\section{LOSSY BUILDINGS WITH SMALL APERTURES}

For electromagnetic sources at VHF impinging on a practical building size, the box represented in figure 2 will be electrically iarge, and thus, the methods outlined in section 2 will be very time consuming, if not impossible. In this section, a different approach to solving the problem of large cavities is considered. In addition, apertures representing doors or windows, and the case of non-perfectly conducting building walls are also included. For the sake of simplicity, only the small aperture in the form of a horizontal narrow slot in an infinite ground plane as shown in figure 6 is studied first.

The approach is based on an application of the equivalence principle [7] and the use of a generalized network formulation [8]. By this approach the small aperture can be replaced by an equivalent magnetic current defined by:

$$
\bar{M}=\hat{y} \times \bar{E}_{a},
$$

FPrivate communication, D.K. Cheng, Syracuse University, Syracuse, New York. 
where $\hat{y}$ is the unit vector in the direction of propagation of the incident wave and $\bar{E}_{a}$ is the electric field at the aperture.

The field in region a of figure 6 is that produced by the incident wave and that due to $\bar{M}$ over the aperture with the aperture covered by a perfect conductor. The field in region $b$ is that produced by $-\bar{M}$ over the aperture with the aperture covered by a perfect conductor. The basic problem here is the determination of $\bar{M}$, with the boundary condition that the tangential component of the magnetic field is continuous across the aperture. This eventually leads to another integral equation involving the unknown $\bar{M}$. The technique similar to that presented in Section 2.2 namely, approxinating $\bar{M}$ by $\Sigma V_{N} \bar{M}_{n}$ with unknown coefficients $V_{n}$, may then be utilized.

For narrow slots of a length not greater than three-quarters of a wavelength, there is evidence that $\bar{M}$ in the slot can be closely approximated by an equiphasal half-cosine wave [9]. This suggests that we may simplify the problem at hand considerably by using a one-term expansion for $\bar{M}$ and write

$$
\begin{aligned}
\bar{M} & =V \bar{M}_{0} \\
& =\hat{x} V \cos \left(\pi x / L_{x}\right)
\end{aligned}
$$

where $L_{x}$ is the length of the slot under the condition $L_{x} \leqslant 3 / 4 \lambda$. The matrix equation is then reduced to a single scalar equation representing the generalized $0 \mathrm{hm}$ 's law:

$$
\left(\gamma^{a}+\gamma^{b}\right) V=I
$$

where $V$ is a generalized voltage to be determined, $\gamma^{a}$ and $\gamma^{b}$ are generalized admittances (in the slot-aperture) of the half-space and the cavity respectively, and $I$ is the generalized current source given by:

$$
I=2 \int_{\text {apert. }} \bar{M}_{0} \cdot \bar{H}_{i} \mathrm{ds},
$$

with $\bar{H}_{j}$ representing the incident magnetic field over the slot-aperture in free space. 
In (34) the generalized half-space admittance in region a is [8].

$$
Y^{\mathrm{a}}=-2 \int_{\text {apert. }} \bar{M}_{0} \cdot \bar{H}\left(\bar{M}_{0}\right) d s
$$

where $\bar{H}\left(\bar{M}_{0}\right)$ is the magnetic field produced and radiated to free space by $\bar{M}_{0}$. In region $b$, the admittance may be expressed in terms of the mode characteristics in the cavity [9],

$$
Y^{b}=-j \sum_{i} A_{i}^{2} Y_{i} \cot k_{i} d
$$

where $Y_{i}$ and $k_{j}$ denote, respectively, the characteristic admittance and the wavenumber of the $i$ th mode, and the coefficient $A_{j}$ is given by:

$$
A_{i}=\int_{\text {apert. }} \bar{M}_{0} \cdot \hat{y} \times \bar{e}_{i} d s,
$$

with $\bar{e}_{j}$ being the normalized ith-mode transverse electric-field vector.

If there exists only one transmission mode in the waveguide in region $b$; that is, if all modes except the $i=1$ mode are cut-off, then $a 11 Y_{j}$ and $k_{j}$ are inaginary except $Y_{1}$ and $k_{1}$. We write [9a]

$$
\begin{aligned}
& Y_{i}=j \beta_{i} \quad(i \neq 1) \\
& k_{i}=-j \alpha_{j} \quad(i \neq 1) .
\end{aligned}
$$

For $i=1, y_{1}$ is the (real) characteristic admittance of the dominant mode, and

$$
k_{1}=\beta_{1}-j \alpha_{1},
$$

where $\beta_{1}$ is the phase constant which is related to the guide wavelength, igl. of the dominant mode

$$
\beta_{1}=2 \pi / \lambda_{g}
$$

and $\alpha_{1}$ is the attenuation constant due to power loss in cavity walls. 
Based on the above, we can construct a generalized equivalent network for the cavity-backed slot-aperture shown in figure 6 . The network consists of a current source I in parallel with $\mathrm{y}^{\mathrm{a}}$, together with

$$
j B=j \sum_{i \neq 1} A_{i}^{2} B_{i} \operatorname{coth} \alpha_{i} d
$$

and a terminated transmission line coupled through an ideal transformer with a turns-ratio $A_{1}$. This is given in figure 7 .

In order to study cavity fields, it is essential to consider that the equivalent transmission line is terminated by a nonzero surface impedance

$$
\begin{aligned}
Z_{S} & =R_{S}+j x_{S}=(1+j) \frac{\alpha_{1}}{\sigma} \\
& =(1+j) \frac{\pi f_{\mu}}{\sigma}
\end{aligned}
$$

where $\sigma$ is the conductivity of the cavity material, and $\alpha_{1}$ for the dominant $\mathrm{TE}_{10}$ mode in an $\mathrm{a} x \mathrm{~b}$ rectangular waveguide is given by

$$
\alpha_{1}=\frac{R_{s}}{n b}\left\{\sqrt{1-\left(\frac{\lambda}{2 a}\right)^{2}}+\frac{(\lambda / 2 a)^{2}}{\sqrt{1-\lambda / 2 a)^{2}}}\left[1+\frac{2 b}{a}\right\rfloor\right\}
$$

$\eta$ being the intrinsic impedance of the medium inside the cavity. For air, $n$ is approximately $377 \Omega$.

For cavity walls made of a good conductor the reactive part of $Z_{S}, X_{S}$, has an insignificant effect on the resonant frequency of the cavity and may be neglected. The real part, $R_{S}$, however influences the $Q$ and field calculations in a major way; it must be retained, as was done in figure 7 .

The input admittance of a lossy transmission line of characteristic admittance $Y_{1}$, wavenumber $k_{1}=\beta_{1}-j \alpha_{1}$, length $d$, and terminated in $R_{s}$ is

$$
Y_{\text {in }}=Y_{1}\left(\frac{1+j Y_{1} R_{s} \tan \left(\beta_{1} d-j \alpha_{1} d\right)}{Y_{1} R_{s}+j \tan \left(\beta_{1} d-j \alpha_{1} d\right)}\right)
$$


At natural resonance,

$$
\beta_{1} d=n_{\pi}, \quad n=1,2, \ldots
$$

Equation (46) becomes

$$
Y_{\text {in }}=Y_{1}\left(\frac{1+Y_{1} R_{s} \tanh \alpha_{1} d}{Y_{1} R_{s}+j \tan \alpha_{1} d}\right) \text {. }
$$

The quantity $\alpha_{1} d$ is usually very small for highly conducting walls, and it is safe to approximate tanh $\alpha_{1} d$ by $\alpha_{1} d$ and to neglect the term $Y_{1} R_{S} \tanh \alpha_{1} d$ in comparison with 1 . We have, from (48)

$$
Y_{i n} \simeq \frac{Y_{1}}{r_{d}},
$$

where

$$
r_{d}=Y_{1} R_{s}+\alpha_{1} d
$$

is a dimensionless quantity. Thus at natural resonance, $Y_{i n}$ is purely real.

The input impedance of the low-loss transmission line terminated in $R_{S}$ in the neighborhood of natural resonance is ( $\beta_{1} d$ not exactly equal to $n \pi$ )

$$
Z_{\text {in }}=\frac{1}{Y_{\text {in }}}=R+j X,
$$

where

$$
R=r_{d} / Y_{1}=r_{d} Z_{1}
$$

and

$$
x=z_{1} \tan \beta_{1} d
$$

The $Q$ of the cavity can be obtained from the definition

$$
Q=\frac{\omega}{2 R} \frac{d X}{d \omega}
$$


Using (52) and (53) for the dorninant mode, we have

$$
Q=\frac{\pi d}{r_{d^{\lambda} g l}}\left(\frac{\lambda g l}{\lambda}\right)^{2} \sec ^{2} \beta_{1} d
$$

where the relation

has been used.

$$
\lambda_{g 1}=\lambda\left[1-\left(\frac{\lambda}{2 \mathrm{a}}\right)^{2}\right]^{-1 / 2}
$$

At natural resonance,

$$
d=\frac{n \lambda g 1}{2}, n=1,2, \ldots
$$

Equation (55) becomes

$$
Q=\frac{\pi d}{r_{d} \lambda_{g l}}\left(\frac{\lambda g l}{\lambda}\right)^{2}=\frac{n \pi}{2 r_{d}}\left(\frac{\lambda g l}{\lambda}\right)^{2}
$$

In order to determine the fields in the slot-aperture and inside the cavity, we must find $V$ from (34). In other words we need to find $I, Y_{d}$ and $Y_{b}$. Assuming an incident plane wave with

$$
\bar{H}_{i}=\hat{x}_{0} e^{-j k y}=\hat{x} \frac{E_{0}}{\eta} e^{-j k y}
$$

where $k=2 \pi / \lambda$, I can be evaluated easily for a narrow $L_{x} \times L_{y}$ slot by using (33) and (59) in (35).

$$
I=\frac{4}{\pi n} L_{x} L_{y} E_{0}
$$

The equivalent half-space admittance $Y_{a}$ can be computed from (36) in terms of an electric vector potential $\bar{F}$ and a magnetic scalar potential $\phi_{\mathrm{m}}$ due to the magnetic current $\bar{M}_{0}$. That is,

$$
\gamma^{a}=2 \int_{\text {apert. }} \bar{M}_{0} \cdot\left(j \omega \bar{F}+\nabla \Phi_{m}\right) d s
$$


where

$$
\bar{F}=\frac{\varepsilon_{0}}{4 \pi} \int_{\text {apert. }} \bar{M}_{0} \frac{\exp \left(-j k\left|\bar{r}-\bar{r}^{-}\right|\right)}{\left|\bar{r}-\bar{r}^{\prime}\right|} \mathrm{ds}^{\prime}
$$

and

$$
\phi_{m}=\frac{j}{4 \pi \mu_{0} \omega} \quad \int_{\text {apert. }}\left(\nabla^{-} \cdot \bar{M}_{0}\right) \frac{\exp \left(-j k\left|r-r^{-}\right|\right)}{|\bar{r}-\bar{r}|} \mathrm{ds}^{-}
$$

These integrals have to be evaluated numerically.

The cavity admittance $y^{b}$ is obtained by combining (37), (43), and (49)

$$
Y^{b}=A_{1}^{2} Y_{i n}+j B
$$

It is also necessary to find the turns-ratio $A_{i}$ of the ideal transformer shown in figure 7 for the dominant mode as well as the $A_{i}(i=2,3 . .$.$) for the$ higher-order modes in (43) from (38). For an a $x$ b rectangular waveguide, the normalized ith mode function for $T_{m n}$ modes $(m+n \neq 0)$ is $[10]$,

$$
\begin{aligned}
\bar{e}_{i} & =\sqrt{\frac{\varepsilon_{o m^{\varepsilon}}}{a b}}\left[\left(\frac{m_{\pi}}{a}\right)+\left(\frac{n_{\pi}}{b}\right)^{2}\right]^{-1 / 2} \\
& \cdot\left[\hat{x}\left(\frac{n_{\pi}}{b}\right) \cos \left(\frac{m_{\pi} x^{-}}{a}\right) \sin \left(\frac{n_{\pi} z^{-}}{b}\right)\right. \\
& \left.+\hat{y} \cdot\left(\frac{m_{\pi}}{a}\right) \sin \left(\frac{m_{\pi} x^{-}}{a}\right) \cos \left(\frac{n_{\pi} z^{-}}{b}\right)\right],
\end{aligned}
$$

where

$$
\varepsilon_{\text {om }}= \begin{cases}1, & m=0 \\ 2, & \mathrm{~m}=1,2, \ldots\end{cases}
$$


The normalized ith mode function for $T_{n m}$ modes $(m \neq 0$ and $n \neq 0)$ is [10]

$$
\begin{aligned}
\bar{e}_{i} & =\frac{2}{\sqrt{a b}}\left[\left(\frac{m_{\pi}}{a}\right)+\left(\frac{n \pi}{b}\right)^{2}\right]^{-1 / 2} \\
& \cdot\left[\hat{x}\left(\frac{m_{\pi}}{a}\right) \cos \left(\frac{n \pi x^{\prime}}{a}\right) \sin \left(\frac{n \pi z^{\prime}}{b}\right)\right. \\
& \left.-\hat{z}\left(\frac{n \pi}{b}\right) \sin \left(\frac{n_{\pi} x^{\prime}}{a}\right) \cos \left(\frac{n \pi z^{\prime}}{b}\right)\right],
\end{aligned}
$$

In (65) and (67), $x^{\prime}=x+a / 2$ and $z^{\prime}=z+b / 2$. Substitution of (33), (65) and (67) in (38) and integration yield the values of $A_{j}$ for all TE and TM modes.

The wavenumber, $k_{i}$, of the ith mode can be expressed in terms of its cutoff wavelength $\lambda_{i c}$ and the corresponding cut-off wavenumber $k_{i c}$,

$$
k_{i}= \begin{cases}k \sqrt{1-\left(\lambda / \lambda_{i c}\right)^{2}}, & \lambda<\lambda_{i c} \\ -j k_{i c} \sqrt{1-\left(\lambda_{i c} / \lambda\right)^{2}}, & \lambda>\lambda_{i c}\end{cases}
$$

where

$$
k_{i c}=\frac{2 \pi}{\lambda_{i c}}=\sqrt{\left(\frac{m \pi}{a}\right)^{2}+\left(\frac{n \pi}{b}\right)^{2}}
$$

The characteristic admittance for the ith mode is

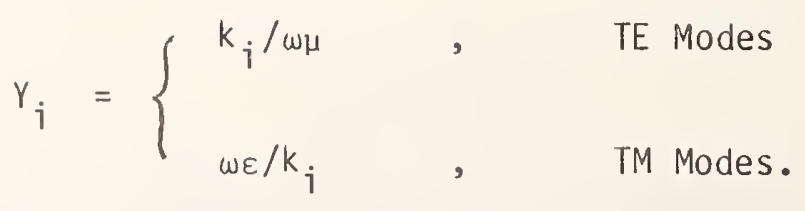

Having found the values of $A_{j}, k_{j}$ and $\gamma_{j}$ for the dominant and higher-order TE and TM modes, we are then in a position to determine $\gamma^{b}$ from (64) in conjunction with (43) and (49). With $\gamma^{a}$ and $\gamma^{b}$ known, the generalized voltage $V$ can be found from (34) using the value of I in (60). 
The transverse electric field inside the cavity for the dominant $T E_{10}$ mode is

$$
\bar{E}_{1 t}=\hat{z} V A_{1} C \cos \left(\frac{\pi x}{a}\right) \frac{\sin k_{1}(d-z)}{\sin k_{1} d}
$$

where

$$
c=\sqrt{\frac{2}{a b}} \text {. }
$$

The field distribution for the higher-order modes has the following expressions:

$$
\bar{E}_{i t}=-\sum_{i \neq 1} V A_{i} \bar{e}_{i} \frac{\sin k_{i}(d-z)}{\sin k d_{i}} \text {. }
$$

These field distributions can be computed for a rectangular cavity of given dimensions, made of a material with known conductivity and having a slotaperture of specified width and length.

We now present some numerical results for the following aspects of a cavity-backed slot-aperture problem at natural resonance: cavity Q, field amplitude in the slot-aperture, and field distribution inside the cavity. The effects of the cavity material on these results are considered. For computational purposes we choose the following parameters:

$\begin{array}{ll}\text { Frequency } & f=100 \mathrm{MHz} \\ \text { Wavelength: } & \lambda=3 \mathrm{~m} \\ \text { Cavity dimensions: } & \mathrm{a}=0.762 \lambda \\ & \mathrm{b}=0.339 \lambda \\ & \mathrm{d}=\lambda_{\mathrm{g} 1} / 2=0.663 \lambda \\ \text { Aperture dimensions: } & \mathrm{L}_{\mathrm{x}}=0.25 \lambda \\ & L_{y}=0.02 \lambda\end{array}$


The cavity quality factor for the dominant $T E_{10}$ mode, $Q$, is calculated for three different wall materials; namely copper, aluminum and iron, using (58) with $\mathrm{n}=1$, which are given in Table 1 .

\section{Table 1}

Cavity quality factor, Q, for dominant mode at

$$
\text { natural resonance. }(\lambda=3 \mathrm{~m})
$$

\section{Cavity material}

Copper

Aluminum

Iron
Conductivity, $\sigma$

$5.80 \times 10^{7} \mathrm{~s} / \mathrm{m}$

$3.54 \times 10^{7} \mathrm{~s} / \mathrm{m}$

$0.95 \times 10^{7} \mathrm{~s} / \mathrm{m}$
Quality factor, Q

$9.19 \times 10^{4}$

$7.18 \times 10^{4}$

$3.72 \times 10^{4}$

The electric-field distribution in the slot-aperture at $z=0$ is approximately a half-cosine whose normalized amplitudes, $\left|E_{S}\right| / E_{O}$, are calculated in accordance with (73) for the three wall materials and tabulated in Table 2.

\section{Table 2}

Normalized amplitude of electric field in the slot-aperture $(x=y=z=0)$ at natural resonance.

Cavity Material

Copper

Aluminum

Iron
Normalized electric-field

$$
\text { amplitude, }\left|E_{S}\right| / E_{O}
$$

$$
\begin{aligned}
& 8.16 \times 10^{-5} \\
& 10.45 \times 10^{-5} \\
& 20.17 \times 10^{-5}
\end{aligned}
$$

The amplitude of the electric field in the aperture is seen to be several orders of magnitude smaller than that of the incident wave. It is most interesting to note that the field there is almost exactly inversely proportional to the square-root of the conductivity of wall material. 
The amplitude of the electric field inside the cavity at $y=d / 2$ has also been calculated for different wall materials. For the present example, it is several orders of magnitude larger than that at the slot-aperture; it is more than three times that of the incident wave; and it is practically independent of the conductivity of the wall material. Table 3 is included to emphasize these points.

\section{Table 3}

Normalized amplitude of electric field at the cavity center, $x=z=0, y=d / 2$ at natural resonance

Cavity Material

Copper

Aluminum

Iron
Normalized electric-field amplitude, $\left|E_{c}\right| / E_{0}$

Figure 8 shows a plot of $\left|E_{1 t}\right| / E_{0}$ along the center axis $x=z=0$, indicating that it is at least possible to produce a high field strength inside the cavity. Note, however, that the specific numerical results given thus far are good only at the first natural resonance when the dominant $T E_{10}$ mode exists and the slot is not too long. For larger slots, more terms should be added in (33) and the computations will be much more complicated. 


\section{LARGE BUILDINGS AND APERTURES WITH LOSSY GROUNDS---A HYBRID APPROACH}

The analysis given in the preceding section is valid only for small apertures and for the building (cavity) in free space. With buildings of practical sizes for the purpose of storage, the buildings and apertures (doors or windows) are usually large at VIH. Furthermore, the buildings and the exterior transmitters are above an imperfect ground and the building walls may be made of some reinforced material which can hardly be considered as perfectly conducting. In view of the outlines presented in Section 2, a rigorous analysis of a practical three-dimensional model based on the Maxwe11's equations and appropriate boundary conditions is certainly not a simple task. For this reason, a hybrid approach---a combination of equations and measured data---together with some necessary simplifications and practical restrictions is proposed in this section.

The field distribution inside an empty building is considered to be produced by a direct component due to the source being operated outside the building and a scattered component re-radiated by an equivalent set of surface current densities induced on the building walls (including the floor and ceiling) which would exist if the wall surfaces were infinitely thin and perfectly conducting.

The geometry and coordinates used here are shown in figure 9, where the building is hollow, situated on an imperfectly conducting ground, with only one door (aperture) appearing on Plane $m_{1} m_{2} m_{3} m_{4}$ (xz-plane) or Plane 3 . The floor plane $\left(m_{1} m_{2} m_{6} m_{5}\right)$ is designated as Plane 1 , the front $\left(m_{1} m_{4} m_{8} m_{5}\right)$ as Plane 2, the ceiling $\left(m_{4} m_{3} m_{7} m_{8}\right)$ as Plane 4, the back $\left(m_{2} m_{3} m_{7} m_{6}\right)$ as Plane 5 , and the left $\left(m_{5} m_{6} m_{7} m_{8}\right)$ as Plane 6 . There is a vertically polarized signal from a transmitter located at $x=D_{c}, y=-y_{t}$, and $z=h_{t}$, where $D_{c}$ is the door center at floor level as indicated in figure 9. 
The basic equation needed here for calculating the scattered field is the combination of (20), (21), and (22):

$$
\begin{aligned}
E_{S}(\bar{r})= & -\frac{j \omega \mu}{4 \pi} \int_{S} \bar{J}\left(\bar{r}^{\prime}\right) G\left(\bar{r}, \bar{r}^{\prime}\right) d s^{\prime} \\
& +\frac{1}{j \omega 4 \pi \varepsilon} \nabla \int_{S} \nabla^{\prime} \cdot J\left(\bar{r}^{\prime}\right) G\left(\bar{r}, \bar{r}^{\prime}\right) d s^{\prime} \\
= & -\frac{j \omega \mu}{4 \pi} \int_{S} \bar{J}\left(\bar{r}^{\prime}\right) G\left(\bar{r}, \bar{r}^{\prime}\right) d s^{\prime} \\
& -\frac{1}{j \omega 4 \pi \varepsilon} \nabla \int_{S} \bar{J}\left(r^{\prime}\right) \cdot \nabla^{\prime} G\left(\bar{r}, \bar{r}^{\prime}\right) d s^{\prime}
\end{aligned}
$$

After approximating the double integral in (76) with a double sumnation by subdividing each of the six planes into $m \times n$ rectangular patches, assuming a constant unknown value for the surface current density on each patch and expressing the final result into components, we obtain

$$
\begin{aligned}
j 4 \pi E_{x}(x, y, z) & =\omega \mu \sum_{m} \sum_{n} J_{x m n}^{(1)} G_{m n 1} \Delta x \Delta y \\
& +\omega \mu \sum_{m} \sum_{n} J_{x m n}^{(3)} G_{m n 3} \Delta z \Delta x \\
& -\frac{1}{\omega \varepsilon} \sum_{m} \sum_{n}\left\{J_{x m n}^{(1)} \frac{1+j k R_{m n 1}}{R_{m n 1}^{2}}+\left[\left(x-x_{m}^{\prime}\right) J_{x m n}^{(1)}+\left(y-y_{n}^{\prime}\right) J_{y m n}^{(1)}\right] a_{m n n}\right\} G_{m n 1} \Delta x \Delta y \\
& -\frac{1}{\omega \varepsilon} \sum_{m} \sum_{n}\left[\left(y-y_{m}^{\prime}\right) J_{y m n}^{(2)}+\left(z-z_{n}^{\prime}\right) J_{z m n}^{(2)}\right] a_{m n 2} G_{\operatorname{mnn} 2} \Delta y \Delta z \\
& -\frac{1}{\omega \varepsilon} \sum_{m} \sum_{n}\left\{J_{x m n}^{(3)} \frac{1+j k R_{m n 3}}{R^{2}}+\left[\left(z-z_{m}^{\prime}\right) J_{z m n}^{(3)}+\left(x-x_{n}^{\prime}\right) J_{x m n}^{(3)}\right] a_{\operatorname{mn} 3}\right\} G_{m n 3} \Delta z \Delta x .
\end{aligned}
$$




$$
\begin{aligned}
& j 4 \pi E_{y}(x, y, z)=\omega \mu \sum_{m} \sum_{n} J_{y m n}^{(1)} G_{m n 1} \Delta x \Delta y \\
& +\omega \mu \sum_{m} \sum_{n} J_{y m n}^{(2)} G_{m n 2} \Delta y \Delta z \\
& -\frac{1}{\omega \varepsilon} \sum_{m} \sum_{n}\left\{J_{y m n}^{(1)} \frac{1+j K R}{R_{m n 1}^{2}}+\left[\left(x-x_{m}^{-}\right) J_{x m n}^{(1)}+\left(y-y_{n}^{-}\right) J_{y m n}^{(1)}\right] b_{m n 1}\right\} G_{I n n 1} \Delta x \Delta y \\
& -\frac{1}{\omega \varepsilon} \sum_{m} \sum_{n}\left\{J_{y m n}^{(2)} \frac{1+j k R}{R_{m n 2}^{2}}+\left[\left(y-y_{m}^{\prime}\right) J_{y m n}^{(2)}+\left(z-z_{n}^{\prime}\right) J_{z I n n}^{(2)}\right] b_{I n n 2}\right\} G_{m n 2} \Delta y \Delta z \\
& -\frac{1}{\omega \varepsilon} \sum_{m} \sum_{n}\left[\left(z-z_{m}^{-}\right) J_{z m n}^{(3)}+\left(x-x_{n}^{-}\right) J_{x m n}^{\left({ }^{3}\right)} b_{m n 3}\right] \Delta z \Delta x,
\end{aligned}
$$

and

$$
\begin{aligned}
& j 4 \pi E_{z}(x, y, z)=\omega \mu \sum_{m} \sum_{n} J_{z m n}^{(2)} G_{m i n 2} \Delta y \Delta z \\
& +\omega \mu \sum_{m} \sum_{n} J_{Z m n}^{(3)} G_{m i n 3} \Delta z \Delta x \\
& -\frac{1}{\omega \varepsilon} \sum_{m} \sum_{n}\left[\left(x-x_{m}^{-}\right) J_{x m n}^{(1)}+\left(y-y_{n}^{-}\right) J_{y m n}^{(1)}\right) c_{m n 1} G_{i n n 1} \Delta x \Delta y \\
& -\frac{1}{\omega \varepsilon} \sum_{m} \sum_{n}\left\{J_{z m n}^{(2)} \frac{1+j k R_{I n n 2}}{R_{m n 2}^{2}}+\left(\left(y-y_{m}^{\prime}\right) J_{y m n}^{(2)}+\left(z-z_{n}^{\prime}\right) J_{z m n}^{(2)}\right] c_{m n 2}\right\} G_{m n 2} \Delta y \Delta z \\
& -\frac{1}{\omega \varepsilon} \sum_{m} \sum_{n}\left\{j z_{z m n}^{(3)} \frac{1+j k R_{m n 3}}{R_{m n 3}^{2}}+\left(\left(z-z_{m}^{-}\right) J_{z m n}^{(3)}+\left(x-x_{n}^{-}\right) J_{x m n}^{(3)}\right) c_{m n 3}\right\} G_{m n 3} \Delta z \Delta x,
\end{aligned}
$$


where

$$
\begin{aligned}
& a_{m n 1}=\left(x-x_{i n}^{-}\right) s_{1}, \\
& b_{m n 1}=\left(y-y_{n}^{-}\right) s_{1}, \\
& c_{\mathrm{mn1}}=\mathrm{zS}_{1}, \\
& a_{m n 2}=x S_{2}, \\
& b_{m n 2}=(y-y-j) s_{2}, \\
& c_{m n 2}=\left(z-z_{n}^{\prime}\right) s_{2}, \\
& a_{\mathrm{mn} 3}=\left(x-x_{n}^{\prime}\right) S_{3} \\
& b_{m n 3}=y s_{3} \\
& c_{\mathrm{mn} 3}=\left(z-z_{m}^{\prime}\right) s_{3} \\
& S_{i}=\frac{k^{2} R_{m n i}^{2}-3-j 3 k R_{m n i}}{R_{m n i}^{4}} \quad, i=1,2,3 \\
& k=\frac{2 \pi}{\lambda}=\frac{2 \pi}{300} \mathrm{f}_{\mathrm{MHz}}, \\
& \mathrm{f}_{\mathrm{MHz}}=\text { operating frequency in } \mathrm{MHz}
\end{aligned}
$$


and

$$
\begin{aligned}
& x_{m}^{\prime}=(m-1) \Delta x+x_{0} \\
& x_{n}^{\prime}=(n-1) \Delta x+x_{0} \\
& y_{m}^{\prime}=(m-1) \Delta y+y_{0} \\
& y_{n}^{\prime}=(n-1) \Delta y+y_{0} \\
& z_{m}^{\prime}=(m-1) \Delta z+z_{0} \\
& z_{n}^{\prime}=(n-1) \Delta z+z_{0} \\
& m, n=1,2,3
\end{aligned}
$$

$x_{0}, y_{0}$, and $z_{0}$ being the possible distances from which the patches are counted. Note that, for simplicity, we have dropped the subscript s in (77) through (79).

A detailed explanation for (77) is now in order. The quantity $E_{x}$ on the left-hand side represents the $x$-component of the scattered complex field strength inside the building at a specified location ( $x, y, z$ ) contributed by the induced surface currents. In reality, this is supposed to be known as it can be obtained by measurements. Let the amplitude and phase be denoted by $V_{x}$, and $\psi_{x}$ for the measured voltage due to the $x$-component of the total field at $(x, y, z)$. The amplitude of the $x$-component of the total field itself is then $\left|E_{t x}\right|=v_{x} / l_{x}$, where the effective length of the dipole, $\ell_{x}$, is approximately equal to $(0.6 \sim 0.9)$ of the actual dipole length, depending on the frequency. The phase information $\psi_{x}$ can be secured by using a reference antenna and a vector voltmeter. This implies that the $x$-component of the total field is: $E_{t x}=\frac{x}{\ell_{x}} L_{x}$. If $\bar{E}_{i}$ represents the incident field from the source, and $\bar{E}_{S}$ scattered field in (76), the total field becomes

$$
\bar{E}_{t}=\bar{E}_{i}+\bar{E}_{s} \text {, or } \bar{E}_{s}=\bar{E}_{t}-\bar{E}_{i}
$$


The $x$-component of (84) is, therefore,

$$
E_{x}=E_{t x}-E_{i x} \stackrel{\sim}{=} E_{t x}=\frac{v_{x}}{l_{x}} / \Psi_{x},
$$

where $E_{i x}$ has been neglected in view of the fact that the incident field is assumed z-polarized.

Separating (85) into the real and inaginary parts, we have

$$
\operatorname{Re} E_{x}=\frac{V_{x}}{l_{x}} \cos \psi_{x} \text {, }
$$

and

$$
\operatorname{Im} E_{x}=\frac{v_{x}}{l_{x}} \sin \psi_{x} .
$$

The first term on the right-hand side in (77) is the $x$-component field contributed by the $x$-component of $\bar{j}\left(\bar{r}^{-}\right)$on the floor $\left(x y-p l a n e\right.$ where $z^{\prime}=0$, or Plane 1) with $J_{x m n}^{(1)}$ representing the $x$-component of the source concentrated at the center of the $m n^{\text {th }}$ patch in Plane $1, G_{m n 1}=\exp \left(-j k R_{m n 1}\right) / R_{m n 1}$, $R_{m n 1}=\left[\left(x-x_{m}^{-}\right)^{2}+\left(y-y_{n}^{\prime}\right)^{2}+z^{2}\right]^{1 / 2}$, and $\left(x_{m}^{-}, y_{n}^{-}, 0\right)$ representing the location of the $\mathrm{mn}^{\text {th }}$ patch. The second term on the right-hand side is the contribution due to the $x$-component of the surface current, $J_{x m n}^{(3)}$, on Plane 3 $(x z-p l$ ane $)$ where $G_{m n 3}=\exp \left(-j k R_{m n 3}\right) / R_{m n}, R_{m n 3}=\left[\left(x-x_{n}^{\prime}\right)^{2}+y^{2}+\right.$ $\left.\left(z-z_{m}^{\prime}\right)^{2}\right]^{1 / 2}$. The first two terms on the right-hand side of (77) are, therefore, directly from the first term of (76).

The remaining three terms in (77), derived from the second term of (76) after performing the necessary mathematical operations of diveryence and gradient, represent contributions from various components of the surface currents on Planes 1, 2 (yz-plane), and 3 (zx-plane). Similar explanations for the various terms in (78) and (79) can also be made.

In (78), we note, for the left-hand side, that

$$
E_{y} \tilde{=} \frac{V_{y}}{l_{y}} / \psi_{y},
$$


yielding

and

$$
\operatorname{Re} E_{y}=\frac{V_{y}}{\ell_{y}} \cos \psi_{y}
$$

$$
\operatorname{Im} E_{y}=\frac{V_{y}}{l_{y}} \sin \psi_{y}
$$

where $V_{y}$ and $\psi_{y}$ are respectively the measured amplitude in volts and phase in degrees due to the $y$-component of the total field, and $\ell_{y}=\ell_{x}{ }^{*}$ Once again, $E_{i y}$ has been omitted.

In (79), we note, for the left-hand side, that

$$
E_{z}=E_{t z}-E_{i z}=\frac{V_{x}}{l_{z}} / \psi_{z}-E_{i z}
$$

where $V_{z}$ and $\psi_{z}$ are the respective measured voltage amplitude in volts and phase in degrees due to the total z-component of the total field, $\ell_{z}=\ell_{x}$, and the z-component of the incident field above a lossy ground is approximately given by:

$$
E_{i z}=\left\{1+R_{v} \exp \left[-j k\left(r_{i}-r\right)\right]\right\} \frac{\sqrt{30 P G}}{r} \exp (-j k r) \exp \left(j k r_{0}\right),
$$

where

$P=$ input power to the transmitter, in watts,

$G=$ transmitting antenna gain (numeric)

$r=$ distance between the transmitter at $\left(D_{c},-y_{t}, h_{t}\right)$ and the measurement point at $\left(x, y, h_{m}\right)$

$=\left[\left(x-D_{c}\right)^{2}+\left(y+y_{t}\right)^{2}+\left(h_{m}-h_{t}\right)^{2}\right\}^{1 / 2}$, in meters,

$r^{\prime}=$ distance between the transmitter image and the measurement point

$=\left[\left(x-D_{c}\right)^{2}+\left(y+y_{t}\right)^{2}+\left(h_{m}+h_{t}\right)^{2}\right]^{1 / 2}$, in meters.

$h_{m}=$ feed point of the measuring antenna above ground, in meters,

$r_{0}=$ distance between the transmitter and the reference antenna, in meters,

$R_{V}=$ Vertically polarized ground reflection coefficient

$$
=\left(\cos \theta-n^{-}\right) /\left(\cos \theta+n^{-}\right) \text {, }
$$

and

$$
\theta=90^{\circ}-\tan ^{-1}\left[\left(h_{1 n}-h_{t}\right) / \sqrt{\left(x-D_{c}\right)^{2}+\left(y+y_{t}\right)^{2}}\right] .
$$


In (92), $n^{\prime}$ is a complex factor involving the ground constants, which may be determined empirically by measuring the z-component field strength called $E_{z}^{\prime}$ at a convenient point. This convenient point may be at the door aperture $\left(D_{c}, D, h_{m}\right)$ when the door is electrically large. Thus,

$E_{z}^{\prime}=\left|E_{z}^{\prime}\right| / \psi_{z}^{\prime}=\left[1+R_{v}^{\prime} \exp \left(-j k r_{i}^{\prime}+j k r^{\prime}\right)\right] \frac{\sqrt{30 P G}}{r^{\prime}} \exp \left(-j k r^{\prime}\right) \exp \left(j k r_{0}\right)$,

where

$$
\begin{aligned}
& r^{\prime}=\sqrt{y_{t}^{2}+\left(h_{m}-h_{t}\right)^{2}}, \\
& r_{i}^{\prime}=\sqrt{y_{t}^{2}+\left(h_{m}+h_{t}\right)^{2}} .
\end{aligned}
$$

From (94) an approximate value for $R_{v}$ (here called $R_{v}^{-}$) at this particular convenient point may be determined. The angle $\theta$ at this particular point becomes

$$
\theta^{-}=900-\tan ^{-1}\left[\left(h_{m}-h_{t}\right) / y_{t}\right)
$$

Substituting (96) and $R_{v}^{\prime}$ obtained from (94) into (92), we can compute

$$
n^{-}=\cos \theta^{-}\left(1-R_{\hat{v}}\right) /\left(1+R_{\hat{v}}\right) \cdot
$$

With $n^{\prime}$ so computed, the ground reflection coefficient $R_{v}$ for a general measurement location can be determined from (92) and (93).

Note that in (77) to (79), the corresponding contributions from Planes 4 , 5 , and 6 have been ignored in order to reduce the number of equations involved. This can be justified as long as the measurement data to be used for $E_{x}, E_{y}$, and $E_{z}$ are taken from the region near the origin $m_{1}$ (see figure 9). Of course, when the measurement data taken from the region near the corner $\mathrm{m}_{2}$ are to be used, Plane 5 (back) will be more important than Plane 2 (front). Under this circumstance, we replace in (77) to (79) $\mathrm{J}_{\mathrm{y} \text { min }}^{(2)}$ by

$J_{y m n}^{(5)}, \quad J_{z m n}^{(2)}$ by $J_{z m n}^{(5)}, a_{m n 2}$ by $a_{m n 5}=(x-w) s_{5}, b_{m n 2}$ by $b_{\operatorname{mn} 5}=\left(y-y_{4}-1\right) s_{5}, c_{\min 2}$ by $c_{\operatorname{mn} 5}=\left(z-z_{n}^{\prime}\right) S_{5}, S_{2}$ by $S_{5}=\left(k^{2} R_{\operatorname{mn} 5}^{2}-3-j 3 k R_{\min 5}\right) / R_{\min 5}^{4}$, and $R_{\min }$ by $R_{m n 5}=\left((x-W)^{2}+\left(y-y_{m}^{\prime}\right)^{2}+\left(z-z_{n}^{-}\right)\right]^{1 / 2}$, where $W$ is the width of the building (see figure 9 ). 
When the measurement data are taken from spaces where contributions from all the planes are considered equally important, the contributions by $\mathrm{J}_{\mathrm{xmn}}^{(4)}$ and $\mathrm{J}_{\mathrm{ymn}}^{\left({ }^{4}\right)}$ from Plane 4 (ceiling), $\mathrm{J}_{\mathrm{ymn}}^{(5)}$ and $\mathrm{J}_{\mathrm{zmn}}^{(5)}$ from Plane 5 (back), and $\mathrm{J}_{\mathrm{Zmn}}^{\left({ }^{6}\right)}$ and $\mathrm{J}_{\mathrm{x} \text { mn }}^{\left({ }^{6}\right)}$ from Plane 6 (1eft) can be easily added to (77) through (79), with similar definitions for $a_{m n i}, b_{m n i}, c_{m n i}, R_{m n i}$, and $S_{m n i}, i=4,5$, and 6. Of course, the number of unknowns will then be doubled. When the assumption made in (77) through (79) is justified, there will be six different complex surface currents with two from each plane, yielding $12 \mathrm{mn}$ real unknowns. The left-hand side of (77) through (79) gives six data values (ReE $i$ and $\operatorname{ImE}_{j}, i=x, y$, and $z$ ) at each measurement point. Thus, if $p$ is designated as the number of locations at which measurements of three orthogonal components (both amplitude and phase) are made, there will be $6 p$ equations. Then, we require

$$
6 p \geqslant 12 \mathrm{mn} \text { or } p \geqslant 2 \mathrm{mn} \text {, }
$$

in order to be able to solve for the system of equations by matrix inversion. In (98), we have assumed that the same number of patches ( $\mathrm{mn}$ ) are considered for each of the three planes. On the other hand, should a different number of patches be taken into account for different planes, then we have,

$$
6 p \geqslant 4\left[m(1)_{n}(1)+m^{(2)} n^{(2)}+m^{(3)} n^{(3)}\right],
$$

or

$$
p \geqslant \frac{2}{3}\left[m^{(1)} n^{(1)}+m^{(2)} n^{(2)}+m^{(3)} n^{(3)}\right],
$$

where $m(i)$ and $n(i)$ are the number of patches considered from Plane $i$, $i=1,2$, and 3 . 
It should be emphasized that although (77) through (79) are important for computing piecewisely the unknown surface currents with a set of measurement data taken at some points near the planes of interest, the same equations are equally useful for computing the field strengths at points other than those data points as soon as the unknown surface currents are determined. In this latter process, the computation is straightforward and does not involve matrix inversions. For this reason, this latter computation is designated as the forward computation.

Naturally, the larger the number of patches to be considered at a time, the more accurate are the numerical results. The actual number of patches used is ultimately determined by compromising the required accuracy and the computing capacity and cost. Since there is no a priori knowledge about the accuracy of the results, we recominend to begin with a sinall number of in and $n$, and then proceed to obtain a solution for the surface currents. These currents can, in turn, be used to perform a forward computation, also using (77) through (79), for the field at different locations where the measured data are also available. A comparison of the computed and measured results on fields should reveal the degree of accuracy by the procedure so outlined. If the accuracy is at an acceptable level, the originally assumed sinall number for $m$ and $n$ is considered satisfactory. Otherwise, a large number for $m$ and $n$ has to be used to improve accuracy.

This process may be verified by on-site measurements if a small computer with reasonable capacity is available.

In principle, whenever the equal sign holds in (98) or (99), a unique solution is always obtainable. In practice, however, we do prefer to have $p$ larger than the minimum required such that the number of equations is larger than the number of unknowns. Under this condition, the solution obtained in the sense of least square fit will minimize large deviations which may result because of the potential errors contained in the measured data. 
With an accurate and approximate set of piecewise solutions for the surface currents obtained from (77) through (79), we then use them to compute the field strength everywhere else inside the building so that the electromagnetic environment of the entire building may be assessed. In general, the space near the door (when it is open) for a large building and door should experience relatively high field strengths. Therefore, careful and regular measurements should be made for the space close to Planes 2, 3, and 5 in order to obtain more accurate results for the surface currents on Planes 1, 2, 3, and 5 near the door, which are important to compute the field strengths for the front space near the door. With such a hybrid analytical procedure one can avoid a large scale of tedious and systematic measurements, which would normally be required. Sample numerical results using this hybrid procedure are given in Section 7. 


\section{MEASUREMENT REQUIREMENTS AND PROCEUURES}

The equipment required for measuring the field strengths consists of three orthogonal dipoles, a pole for supporting the antennas, an antenna selector switch box, a vector voltmeter, a three-channel digital voltmeter/printer, and a reference antenna (monopole), connected as given in figure 10. A movable platform on which the antennas and the selector switch box are mounted is also required. The digital voltmeter/printer prints out three amplitudes in volts and three phases in degrees.

The measurement procedures may be outlined as follows:

(1) Select an origin for the building as shown in figure 9. Identify $x, y$, and $z$ directions.

(2) Measure the building width $W$, depth $D$, and height $H, a l l$ in meters. These may be obtained from the base where the building is being investigated. When $H$ is larger than 5 meters, it may be ignored since the contribution from Plane 4 (ceiling) may be deleted.

(3) Measure the width and height in meters of the door. Determine $D_{C}$ (see figure 9) in meters.

(4) Divide the floor (Plane 1) into regular grids by drawing straight lines parallel to the $x$ - and $y$-axes such that $\Delta x$ and $\Delta y$ are $\lambda / 4$ or less, where $\lambda$ is the operating wavelength. Let $\Delta x=\Delta y$ although they are not required to be equal in principle. Record $\Delta x$ and $\Delta y$ in meters. Observe the intersecting points. Also choose $\Delta z$.

(5) Connect the measurement equipment as shown in figure 10, with one antenna in $x$-direction, another in $y$-direction, and a third in $z$ direction (vertical).

(6) Record the feed height $h_{m}$ of the measurement antennas above the floor, in meters.

(7) Set the transmitter outside the building at $x=D_{C}, y=-y_{t}$, and $z$ $=n_{t}$. Record $y_{t}$ and $n_{t}$ in meters. 
(8) Set the reference antenna at a convenient location outside the building. Record the distance $r_{0}$ in meters between the transmitter and the reference antenna.

(9) Record the frequency $f$ in $M H z$ of the transmitting antenna which is assumed to be vertically polarized $\left(E_{i z}\right.$ only), the input power $P$ in watts, and the antenna gain $G$ (numeric).

(10) Determine $x_{0}, y_{0}$, and $z_{0}$ in meters required in (83). This is necessary because of the size of the moving platform on which the measurement antenna is mounted.

(11) Mark the first intersecting point [obtained in (4)] near the origin where measurements are possible as position $(1,1)$. Number the next intersecting points toward the +x-direction as $(2,1),,(3,1)$ etc. to form the first row. Similarly, number the next intersecting points toward the ty-direction as $(1,2),(1,3)$, etc. to form the first column.

(12) Make as many columns as possible between Planes 2 and 5. Make as many rows as possible up to $y=D / 2$.

(13) One person is assigned to the transmitter to turn on and off of the transmitting signal as instructed.

(14) Another person is assigned to record the signal from the printer.

(15) A third person is assigned to align the feed point of the orthogonal dipoles to position $(1,1)$. Once done, he asks the transmitter operator outside the building to key on the signal, walks away from the measuring antennas, and asks the printer operator to record the amplitudes and phases of the three component voltages $\left(V_{x}, V_{y}, V_{z}, \psi_{x}, \psi_{y}, \psi_{z}\right)$ in volts and degrees respectively, together with the measurement position. 
(16) Once the signals are recorded, the person assigned to move the measuring antennas asks the transmitter operator to turn off the signal. He then moves the measuring antenna to position $(2,1)$, signals the transmitter to be turned on, and repeats the process.

(17) Take and record at least 10 rows and 5 columns each along Planes 2 and 5 .

(18) Take and record a few points around the center of the building ( $x=$ $w / 2, y=0 / 2)$.

(19) Take and record a few points at the door aperture $(y=0)$.

(20) Raise the antenna feed point by $\lambda / 4$, and repeat the same process starting position $(1,1)$. 


\section{COMPUTING REQUIREMENTS AND PROCEDURES}

This section contains a discussion of four computer programs used to solve (77), (78), and (79) given in section 4. The main part of the discussion deals with two FORTRAN IV programs, one that computes the currents, $\bar{J}$, from the known measured field strengths, and another that computes field strengths, $\bar{E}$, from given currents. A second pair of programs, using HPL language, is based on the FORTRAN programs but is designed for a smaller desktop computer.

When computing currents, a $6 p$-by-12mn matrix is formed, which can become large if $p, m$, and $n$ are large ( $p, m$, and $n$ are defined in section 4 ). Thus the computer's central memory storage capacity requirement will be large. For example, let $m$ and $n$ be equal to 2 . This defines $a 2 \times 2 \times 2$-point measurement cell which we consider to be the smallest data set that will produce meaningful results. The matrix size is $48 \times 48$ elements. To compute the currents in $\mathrm{Pl}$ anes 1,2 , and 3 requires a central memory containing approximately 25,000 storage locations. If $m$ and $n$ are equal to 6 (i.e., a $6 \times 6 \times 2$-point data set), the matrix size is $216 \times 120$ elements and approximately 250,000 storage 1ocations are required. For a computer that is too small to execute this program even for the smallest (48 x 48) matrix, the program is divided into two parts as described in section 6.3 below.

When computing field strength, no matrix operations are required, and the required amount of storage does not strongly depend upon the data set size. Approximately 20,000 storage locations are adequate for a $6 \times 6 \times 2$-point data set.

The actual computer size required will depend upon the individual computer system's architectural details such as compiler design, word length capacity, exponent range, on-line storage capability, etc. The large computer used to execute these programs at NBS has the following characteristics:
a. $\quad$ NOS operating system
b. $400 \mathrm{~K}$ words (octal) central memory capacity
c. 60-bit word size
d. \pm 322 exponent range
e. $\quad \sim 10^{7}$ instructions per second 


\subsection{CURRENT Program (FORTRAN)}

The FORTRAN program used to solve (77), (78), and (79) for the induced currents in Planes 1, 2, and 3 is given in Appendix A, section A.1. In its present form, this program contains a large amount of redundancy as a result of the decision to mimic the original algebraic equations of the problem. This allowed the program to be proof-read for accuracy by project personnel who have only a casual acquaintance with FORTRAN language. For a large program such as this one, much debugging cost can be saved by this approach. A more efficient program could be written, but little overall machine savings would result because the vast majority of computer memory is consumed by the $6 p$-by-12mn matrix. The program as shown is considered adequate for the present purposes.

The following background discussion will be helpful for understanding this program.

Equations (77), (78), and (79) can be written in matrix notation as follows:

$$
[\bar{E}]=[A][\bar{J}] \text {, }
$$

where $[\bar{E}]$ is a u-vector, $[\bar{J}]$ is a v-vector, and $[A]$ is a $u-b y-v$ matrix. In our case, $[\bar{E}]$ is a $6 p$ - element column matrix $(u=6 p)$,

$$
[\bar{E}]_{p}=\left[\begin{array}{c}
E_{x}(x, y, z) \\
E_{y}(x, y, z) \\
E_{z}(x, y, z)
\end{array}\right]
$$

and $[\bar{j}]$ is a $12 \mathrm{mn}$ - element column matrix $(v=12 \mathrm{mn})$, The elements in matrix [A] are the coefficients of the currents $\vec{j}$ as found in (77), (78) and (79).

The solution of (100) for the currents, [j] , makes use of the "QR factorization" [11] of matrix [A], thus converting (100) into a form that can be solved directly by back-substitution [12]. In the QR factorization method. an orthogonal matrix, [Q], and an upper triangular matrix. [R], are formed such that

$$
[Q][R]=[A] \text {. }
$$


An orthogonal matrix has the property,

$$
[\mathrm{Q}]^{\top}[\mathrm{Q}]=[\mathrm{I}] \text {, }
$$

where $[Q]^{\top}$ is the transpose of $[Q]$, and $[I]$ is the unit (or identity) matrix,

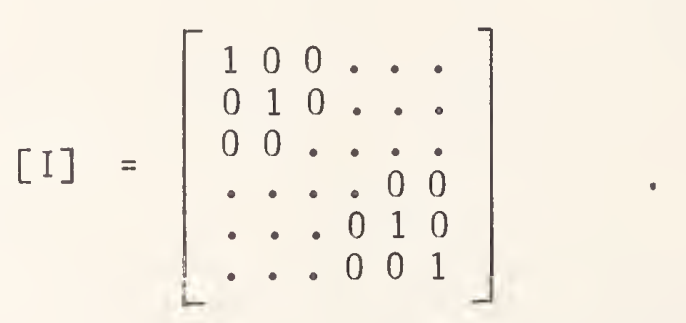

An upper triangular matrix has the form,

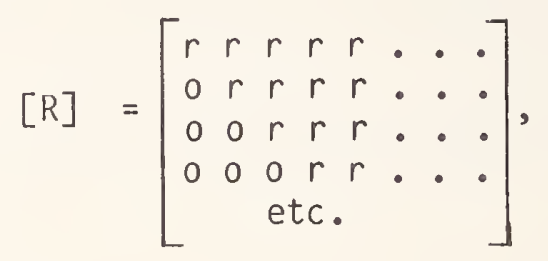

in which elements $r_{i j}$ are equal to zero when $i>j$.

From (102) and (103)

$$
[R]=[Q]^{\top}[A] \text {. }
$$

Multiplying (100) through by $[Q]^{\top}$, and applying (106) gives

$$
\begin{aligned}
{[Q]^{\top}[\bar{E}] } & =[Q]^{\top}[A][\bar{J}] \\
& =[R][\bar{J}] .
\end{aligned}
$$

Because [R] is upper triangular, (107) is easily solved for the elements of $[\bar{j}]$ by back-substitution, starting with the last $(12 \mathrm{~nm}-\mathrm{th})$ element. Thus,

$$
J_{12 m n}=\frac{(q E) 6 p}{r_{6 p, 12 m n}}
$$


where $J_{12 m n}$ is the last element in $[\bar{J}],(q E)_{6 p}$ is the last element in $[Q]^{\top}[\bar{E}]$, and $r_{6 p}, 12 \mathrm{mn}$ is the corresponding element in [R]. Having found $\mathrm{J}_{12 \mathrm{mn}}$, we next find element $\mathrm{J}_{12 \mathrm{mn}-1}, \mathrm{~J}_{12 \mathrm{mn}-2}$, and so on until all elements in $[\bar{j}]$ have been found.

The solution thus obtained is a least square solution [13] of the system of equations.

The program as shown in section $A .1$ is based upon the following assumptions

a. The field strength measurement data are stored in a computer file in the specific ordered sequence as shown in Tables 1 and 2, section 7 . Each data value occupies a specific position in the data grid that corresponds, point-for-point, with the measurement grids discussed in section 5 .

b. The intervals $\Delta x$ and $\Delta y$, between the data points, are constant in both the $X$ - and $Y$-directions. They are designated DX and DY in the program, and may be either equal or not equal to each other.

c. Measurement data are obtained at two levels above Plane 1 (floor). The lower level height above Plane 1 is identified as ZSTART, and the upper level is $D Z$ above ZSTART.

d. Out of the complete set of measurement data, the number of data point locations in the $x$-direction at the lower (first) level that are used in these computations is 2. This is identified as $\operatorname{NXTOTH}(1)=2$, which freely translated, reads, "the TOTal Number of $\underline{X}$-direction data locations in the first level Held for computation use is equal to two." Similarly, $\operatorname{NXTOTH}(2)=\overline{2}$ identifies that there are two X-direction data locations at the upper (second) level used in the computations. NYTOTH(1) and NYTOTH(2) refer to the number of $Y$-direction data locations in the first and second levels, respectively, used in the computation. Bear in mind that there are six data numbers at each location; viz., two each (the real and imaginary parts) in the three $(X, Y$ and $Z$ ) orthogonal directions.

e. Only the currents in Planes 1,2 and 3 are calculated. This is in keeping with the discussion in section 4 . 
Assumption a allows the data at any desired grid location to be used in the computation. The computer selects the desired data according to program statements beginning with the letters IXH and IYH. This is discussed further below.

The consequence of assumption $b$ is that the coordinates of the data used in the computations are calculated by the computer for use in the program rather than being provided to the computer as input data. The formulae for coordinate computation has the form,

$$
x=(m-1) \Delta x+x(\text { start }),
$$

where $m$ is an integer-index $(m>0)$ that specifies the desired data point. Assumptions $c$ and $d$ are arbitrary and can easily be changed if desired. We have executed this program for data sets as large as six (6) in each of the $X$ - and $Y$-directions ( $i . e ., 6$ data point locations in the $X$-direction, 6 in the $Y$-direction, and 2 in the Z-direction). If measurement data had been taken at more than two levels, a simple modification of the program would allow it to be used. However, the maximum size of the data set is limited by the computer's central memory storage capacity as stated above.

The consequence of assumption e is that the program simply omits statements that would be used to calculate currents in Planes 4, 5, and 6 . These statements can be added as desired, using the same basic code as used for Planes 1, 2, and 3. We have, for example, a second program, like the one shown in section $A .1$, that calculates currents in Planes 1,3 , and 5 . For discussion, the program may be divided into five parts as follows:

1. Lines 1 through 56 contain basic information such as variable definitions, dimension statements, values of constants, and selectable parameter values.

2. Lines 57 through 237 accomplish the computation of the elements of coefficients matrix [A]. These computations, for the most part, involve the geometry of the problem, i.e., the locations of the currents relative to the locations of the points at which field strength data were measured.

3. Lines 238 through 244 deal with subroutine CQRDCT by which the QR factorization of matrix [A] is accomplished. 
4. Lines 245 through 309 construct matrix $[\bar{E}]$ by reading in the measurement data from the data file and modifying it according to (90), et seq., of section 4 .

5. Lines 310 through 359 (the end of the program) accomplish the solution of (100) for the currents by use of subroutine CQRSLT, which is the back-substitution procedure for solving (107).

A finely detailed discussion of this program will not be given because familiarity with FORTRAN programming is assumed on the part of the reader. However, certain features should be briefly described. These are grouped according to the five parts of the program listed above.

Part 1 (1ines 1 through 56). The statements that begin with the letters IXH and IYH determine which data are used in the computation of currents. They do this by defining an index that is used at various places throughout the program to select the desired data values (from the data file) and to compute their coordinate locations.

These statements have the form,

$$
\operatorname{IXH}(a, b)=c,
$$

where $a, b$ and $c$ are integers. The letter I means that this is an index. $x$ means it pertains to the $X$-direction. $H$ means that the index is held in memory for use as needed. Integer $a$ is an ordinal number signifying the sequential order of this index. For example, if $a=1$, then this is the first index of its particular kind that will be used in later do-loops. Integer b refers to measurement grid level. If $b=1$, the index refers to data in the first (1ower) measurement grid; $b=2$ refers to the second (upper) grid. Integer $c$ is an ordinal number that refers to the row or column in the chosen measurement grid where the desired data point is located. For example, if $c=$ 1 in eq. (110), the index refers to the first data point in the x-direction. 
Here are two examples. In words,

$$
\operatorname{IXH}(1,2)=3
$$

reads. "the first data point used by the do-10op from the second (upper) measurement grid is the third point in the $x$-direction in that measurement grid." Similarly,

$$
\operatorname{IYH}(2,1)=9
$$

reads, "the second data point used by the do-loop from the first (lower) measurement grid is the ninth point in the $Y$-direction in that measurement grid."

The user may change the integer $c$ to select any particular sets of data that he desires to be used in the computation of currents. The computer will calculate the coordinates of those data and use them whenever they are required in the program.

As mentioned above, the program printed in section $A .1$ is written to call two data points from each level in each of the $X$ - and $Y$-directions. If more data points are desired, suitable IXH or IYH statements are added. For example, if a third data point is to be added from the first level measurement grid in the $X$-direction, this statement would be added:

$$
\operatorname{IXH}(3,1)=c \text {, }
$$

where $c$ is defined above. In this case the NXTOTH (1) statement must be changed to read

$$
\operatorname{NXTOTH}(1)=3,
$$

since now there are a total of three data points in the $x$-direction that will be incorporated by the proqram. The number to the right of the equals sign in the NXTOTH and NYTOTH statements must be the same as integer a in the corresponding IXH and IYH statements. 
Part 2 (1ines 57 through 237). Coordinates of field locations within the building and of current locations in Planes 1, 2, ánd 3 are calculated in this part of the program. The former are designated by the letters $X, Y$, and $Z$, and the latter by XP, YP, and ZP.

Also calculated here are the constants $a_{m n i}, b_{m n i}, c_{m n i}, G_{m n j}, R_{m n i}$, and $S_{j}$, which are defined in section 4 .

Using this information, the computer calculates current coefficients CJX, CJY, and CJZ. These coefficients are structured by the computer as the elements of matrix [A]. In general, the coefficients are complex and are comprised of several terms. For example, the coefficient of the current $j_{\mathrm{xmn}}^{(1)}$ is, from (77),

$$
\operatorname{cux1_{mn}}=-\left\{\frac{j \omega \mu}{4 \pi}-\frac{j}{4 \pi \omega \varepsilon}\left[\frac{1+j k R_{m n 1}}{R_{m n 1}^{2}}+\left(x-x_{m}^{-}\right) a_{m n 1}\right]\right\} G_{m n 1} \Delta x \Delta y .
$$

However, each coefficient reduces to a single complex number pair.

For $\operatorname{NXTOTH}(1)=2, \operatorname{NXTOTH}(2)=2, \operatorname{NYTOTH}(1)=2$, and $\operatorname{NYTOTH}(2)=2$,

$$
\begin{aligned}
& p=2+2+2+2=8, \\
& m=2, \\
& n=2,
\end{aligned}
$$

and

$$
\begin{aligned}
6 p & =6 \times 8=48, \\
12 m n & =12 \times 2 \times 2=48 .
\end{aligned}
$$

Thus [A] is a 48-by-48 element matrix.

Although this part of the program appears to be lengthy, it actually contains a basic code that is repeated three times, once each for Planes 1, 2 , and 3.

Part 3 (1ines 238 through 244). Subroutine CQRDCT is shown in section A.2. This is a compact form of a public-domain program called CQRDC, which stands for Complex QR Decomposition [14]. CQRDCT was written especially for this project by $P$. N. Swarztrauber [15]. Consult reference [14] for a description of this subroutine.

Notice that the symbol $A$ is used for two different matrices, neither of which is the matrix [A] discussed above. First, it defines a doubly subscripted complex array that contains matrix [A] of (100). Second, it defines the upper triangular matrix [R] of (102) used in the solution of (107). 
Part 4 (1ines 245 through 309). The measurement data is read from the data file (line 288), converted to field strength by dividing the voltage readings by $\ell_{z}$, and corrected for the incident field per (90). The three components of the scattered field are structured as the elements of matrix $[\vec{E}]$.

Part 5 (1ines 310 through 359). Subroutine CQRSLT is shown in section A. 3. This is a compact form of a public-domain program called CQRSL, which stands for Complex QR Solver [14]. CQRSLT was written especially for this project by P. N. Swarztrauber [15]. Consult reference [14] for a description of this subroutine.

The solution to (107), and thus the solution to (100), is written out as the $12 \mathrm{mn}$ elements of matrix [j]. These are designated by the symbol XS.

\subsection{FIELD Program (FORTRAN)}

The FORTRAN program used to solve (77), (78), and (79) for the field strengths $\vec{E}$, given the induced currents, $\bar{j}$, is given in Appendix $A$, section A.2. This proqram employs much that is used in the program listed in section $A .1$, and like that program, it was written for greatest proof-reading ease rather than for greatest efficiency.

The form of the program is based upon the same five assumptions listed in Section 6.1 with the following exceptions:

a. Input data is the current matrix [j], which is stored in a computer file in a specific ordered sequence that corresponds, point-for-point, with the projections of the measurement data locations.

b. The locations of the computed field strengths can be at any arbitrary points chosen by the user.

The discussions of this program is divided into the following four parts:

1. Lines 1 through 54 contain the same basic information discussed in section 6.1 .

2. Lines 55 through 127 read the input data from the computer file that contain matrix $[\bar{j}]$, and prints out the matrix elements for verification.

3. Lines 128 through 289 compute the coefficients matrix [A], and then solve

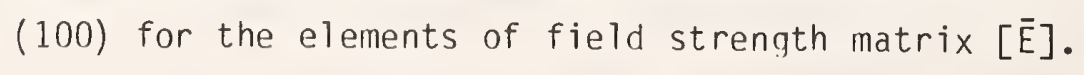

4. Lines 290 through 349 (the end of the program) add the incident field per (90), and print out the computed fields strength at the selected field point (s). 
A brief discussion of this program follows:

Part 1 (1ines 1 through 54). This part of the code is identical to its counterpart in the preceding section, except that the coordinates of the point at which the user desires to compute the field strength are defined as XFP, YFP, AND ZFP. They may be changed at will.

Part 2 ( 7 ines 55 through 127). The input data are those values of $j$ that are selected according to their coordinate locations. These in turn, are determined by the indices IXH and IXY as discussed in section 6.1. The elements of the current matrix [j] that is formed from the input data are defined by the symbol XS.

The coordinates of the current locations in Planes 1, 2, and 3 are calculated by the same code discussesd in section 6.1. They are designated by the letters XP, YP, and ZP.

Part 3 (1ines 128 through 289). Constants $a_{m n i}, h_{m n i}, c_{m n i}, G_{m n i}, R_{m n i}$, and $S_{j}$, and the current coefficients CJX, CJY, and CJZ are calculated by the same code discussed in section 6.1. A major difference in the two programs occurs at this point; namely, this program computes the elements of matrix [A] and solves for the elements of matrix [E] point-by-point without the need to construct matrix [A] in toto. This greatly reduces the central memory size requirements as compared with the program of section 6.1. At this point, the incident field contribution has not yet been added in.

Part 4 (1ines 290 through 349). The incident field strengths are calculated by the same code discussed in section 6.1 , and used to correct the computed fields per (90).

\subsection{CURRENT Program (HPL)}

The HPL program used to solve (77), (78), and (79) for the induced currents in Planes 1, 2, and 3 is given in Appendix B, section B.1. It is divided into two parts so that a small desktop computer can he used. Part 1 computes the elements of the coefficients matrix [A], and performs the QR factorization given by (106) and (107). Part 2 computes the elements of the current matrix [j].

This program is based on the FORTRAN program discussed in section 6.1. It uses the same computational approach, and the same assumptions apply except for assumption a (pertaining to the input data). In the HPL prouralu. the coordinates of the data points that are to be used in the computation are first manually entered in accordance with prompting instructions provided by 
the computer. Later, the field data values are manually entered, again following the computer's prompting. Having obtained the data in this way, the computer calculates the solutions to (77), (78), and (79) by the same methods discussed in section 6.1. These current values and their coordinates are stored on the HPL program tape for subsequent use by the FIELD program described in section 6.4 , below.

A detailed discussion of the design of this program will not be given because (a) familiarity with HPL programming is assumed on the part of the reader, and (b) the general design of the program is closely similar to the corresponding FORTRAN program. Refer to the discussion given in section 6.1 for information on these programs.

Variable assignments are given in section B.2. Instructions for executing the HPL CURRENT program are given in section B.3.

\subsection{FIELD Program (HPL)}

The HPL program used to solve (77), (78), and (79) for the field strength at any arbitrary location within the building, after the currents are obtained, is also given in Appendix B, section B.1. It is based upon the FORTRAN program discussed in section 6.2. It uses the same computational approach, and the same assumptions apply. In the HPL program, the coordinates of the field points are manually entered in accordance with prompting instructions provided by the computer. The current values and their coordinates are obtained from the CURRENT program. (see section 6.3, above).

The general design of this program is closely similar to the corresponding FORTRAN program. Refer to the discussion given in section 6.2 for information on these programs.

Variable assignments are given in section B.2 (same as for HPL CURRENT program). Instructions for executing the HPL FIELD program are given in section $B .3$. 


\subsection{HPL File Structure on Magnetic Tape}

Both the HPL CURRENT and the HPL FIELD programs are on a single magnetic tape. They are structured such that (a) each program may be run independently of the other, or (b) the two programs can be run in uninterrupted sequence. However, the CURRENT program must have been run before the FIELD program can be run alone because the latter requires computed information from the former. Once the CURRENT program has been run and its output stored on the tade, the FIELD program can be run any number of times to compute the field strengths at any number of arbitrary field points. Each of these field computations will pertain to the one set of currents computed by the CURRENT program, of course.

HPL CIJRRENT program, Part 1 , is stored in file \#1 of the magnetic tape. CURRENT program, Part 2, and the FIELD program stored in files \#2 and \#3, respectively. Computed currents and their coordinates from the CURRENT program are stored in file \#4.

\subsection{Print Options for HPL Programs}

The two HPL programs are provided with a means to either permit or prevent the printing out of certain numerical information that is available within the computer. This is accomplished by the use of flags. The user can determine whether or not to print this information by modifying the programs before running them. Some of the printable information is useful primarily as an aid in diagnosing the cause of problems and would not normally be printed out in a routine run. Other information, such as the computed currents $J$, may or may not be needed in hard-copy form. Printing out any information lengthens the time required to run the programs.

The print option applies to the following information. The procedure for setting the print option flags is described below.

1. Flag 1 controls the printing of the six constants.

$\begin{array}{lll}\text { a. } & \text { OMEGA } & =2 * P I * F R E Q, \text { where FREQ }=\text { test frequency } \\ \text { b. } & \text { MU } & =\text { permeability of free space } \\ \text { c. } & \text { EPSILON } & =\text { permittivity of free space } \\ \text { d. } & \text { KAY } & =O M E G A \sqrt{(M U * E P S I L O N)} \\ \text { e. } & \text { OMS4P } & =O M E G A \sqrt{M U /(4 * P I)} \\ \text { f. } & \text { SO4PE } & =1 /(O M E G A * 4 * P I * E P S I L O N)\end{array}$


2. Flag 2 controls the printing of the A coefficients.

3. Flag 3 controls the printing of the quantities $u$ and $v$ defined in section 6.1. In the HPL proqrams, $u$ and $v$ are designated by MA and NA, respectively.

4. Flag 4 controls the printing of the diagonal of the matrix [R].

5. Flag 5 controls the printing of $E_{Z}$, defined by (90).

6. Flag 6 controls the printing of the residual of the least squares approximation involved in the solution of (100).

7. Flag 7 controls the printing of the currents $\mathrm{J}$.

Items 1, 2, 3, 4, and 6, above, are primarily useful for diagnostic purposes, and are not normally printed out. $E_{z}$ (item 5) may be of interest in certain cases. The current J (item 7) may be printed out to obtain an idea of the distribution of the induced currents in the walls of the rectangular box. These currents are, of course, the output of the CURRENT program, and they are input data to the FIELD program. Thus they may be printed out when it is desired to verify that the same set of values appear in both programs.

In the CURRENT program, statements 6 through 12 of Part 1 pertain to these flags. In the FIELD program, statements 6, 7, and 8 apply. To prevent printing, the syntax following the colon is "sfg"; to permit printing, the syntax is "cfg". The program listing given in section B.I show the flags set to prevent printing of all the items lister above. 
The result for a building at a California site is hereby presented. Appropriate input data are given below.

$$
\begin{aligned}
& \text { W }=\text { building width }=8.763 \mathrm{~m} \text {. } \\
& \text { D } \quad \text { building depth }=24.232 \mathrm{~m} \text {. } \\
& \mathrm{H}=\text { building height }>5 \mathrm{~m} \text {. (The ceiling plane may be neglected). } \\
& \text { Door size }=4.267 \mathrm{~m} \text { by } 3.658 \mathrm{~m} \text {. (electrically large). } \\
& D_{C}=\text { door symmetrical point }=3.048 \mathrm{~m} \text {. (The door is not } \\
& \text { symmetrically situated on Plane } 3 \text {, however). } \\
& y_{t}=\text { horizontal distance between the transmitter and the } \\
& \text { door }=9.906 \mathrm{~m} \text {. } \\
& h_{t}=\text { height of the transmitter above ground }=1.219 \mathrm{~m} \text {. } \\
& \text { P = input power to the transmitter }=45 \mathrm{~W} \text {. } \\
& \mathrm{G}=\text { transmitting antenna gain } \cong 0.3 \text {, including the ground effect. } \\
& f=\text { operating frequency of the transmitter }=148.1 \mathrm{MHz} \\
& \lambda=\text { corresponding wavelength }=2.026 \mathrm{~m} \text {. } \\
& h_{m}=\text { height above ground of the feed point of the measuring antenna }= \\
& 1.4478 \mathrm{~m} \text {. or } 1.9050 \mathrm{~m} \text {. (two antenna heights) } \\
& \Delta x=\Delta y=\text { size of the grid }=0.6096 \mathrm{~m} . \Delta z=0.4572 \mathrm{~m} \text {. } \\
& x_{0}=\left\{\begin{array}{l}
\text { distance of Position }(1,1) \text { from Plane } 2=0.9144 \mathrm{~m} \\
\text { distance of Position }(13,1) \text { from Plane } 5=0.5334 \mathrm{~m} .
\end{array}\right. \\
& y_{0}=\text { distance of Position }(1,1) \text { from Plane } 3=1.0668 \mathrm{~m} \text {. }
\end{aligned}
$$


$z_{0}=$ distance from the floor plane to the edge of the first grid on Planes 2 and $5=0$

$r_{0}=$ distance between the transmitter and reference antenna $=9.687 \mathrm{~m}$.

There are 16 rows and 13 columns. The actual measured data are tabulated as follows:

$\underline{\text { Table } 4}$

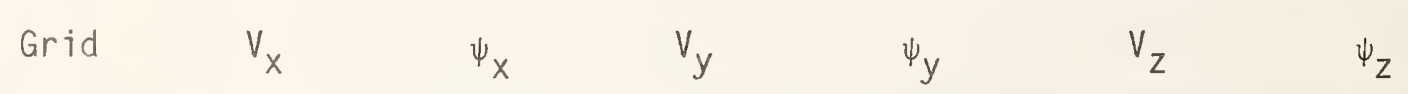

Position in volts in deg. in volts in deg. in volts in deg.

$\begin{array}{lrrrrrr}(1,1) & 0.040 & -171.4 & 0.030 & -46.3 & 0.030 & 107.3 \\ (2,1) & 0.034 & -145.6 & 0.013 & -25.0 & 0.090 & 116.6 \\ (3.1) & 0.009 & -97.4 & 0.022 & 168.5 & 0.146 & 110.1 \\ (4,1) & 0.014 & 41.7 & 0.024 & -104.6 & 0.132 & 101.2 \\ (5,1) & 0.009 & 68.5 & 0.041 & -73.0 & 0.088 & 116.9\end{array}$

$\begin{array}{rrrrrrr}(9,1) & 0.010 & 81 . & 0.014 & -116.3 & 0.010 & -90.4 \\ (10,1) & 0.009 & 95.2 & 0.009 & -175.4 & 0.011 & -157.5 \\ (11,1) & 0.009 & 56.3 & 0.010 & 135.8 & 0.015 & 18.9 \\ (12,1) & 0.006 & 39.7 & 0.005 & 42.4 & 0.011 & 3.0 \\ (13,1) & 0.003 & -24.5 & 0.011 & -14.1 & 0.012 & -158.5\end{array}$




\begin{tabular}{|c|c|c|c|c|c|c|}
\hline$(1,2)$ & 0.025 & 101.8 & 0.030 & -110.6 & 0.043 & -24.0 \\
\hline$(2.2)$ & 0.044 & 118.7 & 0.034 & 87.1 & 0.044 & -3.2 \\
\hline$(3,2)$ & 0.026 & 87.7 & 0.056 & 101.2 & 0.112 & 1.6 \\
\hline$(4,2)$ & 0.010 & -20.5 & 0.025 & 165.4 & 0.131 & -9.7 \\
\hline$(5,2)$ & 0.006 & -131.9 & 0.023 & -154.8 & 0.099 & 5.4 \\
\hline$(9,2)$ & 0.008 & 40.7 & 0.010 & -100.6 & 0.012 & -133.7 \\
\hline$(10,2)$ & 0.009 & 54.7 & 0.007 & 54.6 & 0.027 & 133.2 \\
\hline$(11,2)$ & 0.007 & 116.7 & 0.009 & 132.1 & 0.024 & 42.4 \\
\hline$(12,2)$ & 0.006 & 139.8 & 0.002 & -35.5 & 0.005 & 22.0 \\
\hline$(13,2)$ & 0.005 & 96.3 & 0.009 & -20.6 & 0.020 & 178.1 \\
\hline$(1,3)$ & 0.011 & -23.7 & 0.034 & 167.7 & 0.048 & -112.5 \\
\hline$(2,3)$ & 0.029 & 39.5 & 0.039 & 43.1 & 0.083 & -122.9 \\
\hline$(3,3)$ & 0.018 & 10.2 & 0.047 & 29.4 & 0.127 & -111.3 \\
\hline$(4,3)$ & 0.015 & -111.7 & 0.007 & 22.5 & 0.140 & -106.9 \\
\hline$(5,3)$ & 0.015 & -158.4 & 0.011 & 59.9 & 0.096 & -112.2 \\
\hline$(9,3)$ & 0.019 & -34.4 & 0.003 & 161.2 & 0.039 & -174.4 \\
\hline$(10,3)$ & 0.020 & -27.0 & 0.013 & 81.1 & 0.027 & 120.4 \\
\hline$(11,3)$ & 0.006 & -70.5 & 0.011 & 130.5 & 0.027 & -35.7 \\
\hline$(12,3)$ & 0.008 & -153.5 & 0.002 & -72.3 & 0.022 & -103.7 \\
\hline$(13,3)$ & 0.008 & -82.3 & 0.016 & -37.6 & 0.025 & 166.7 \\
\hline
\end{tabular}




\begin{tabular}{|c|c|c|c|c|c|c|}
\hline$(1,4)$ & 0.015 & -167.6 & 0.041 & 119.0 & 0.046 & 130.2 \\
\hline$(2,4)$ & 0.031 & -74.7 & 0.021 & -15.8 & 0.098 & 127.6 \\
\hline$(3,4)$ & 0.038 & -105.4 & 0.039 & -75.7 & 0.120 & 147.0 \\
\hline$(4,4)$ & 0.026 & -175.5 & 0.025 & -128.6 & 0.114 & 161.7 \\
\hline$(5,4)$ & $0.0 ? 9$ & 110.0 & 0.007 & 69.9 & 0.094 & 148.9 \\
\hline$(9.4)$ & 0.005 & -118.3 & 0.012 & 117.6 & 0.034 & 105.2 \\
\hline$(10,4)$ & 0.010 & -19.3 & 0.015 & 81.7 & 0.023 & 35.1 \\
\hline$(11,4)$ & 0.014 & -4.7 & 0.008 & -147.8 & 0.040 & -73.2 \\
\hline$(12,4)$ & 0.003 & 9.2 & 0.010 & -129.7 & 0.019 & -119.0 \\
\hline$(13,4)$ & 0.004 & -122.6 & 0.012 & -50.9 & 0.030 & 104.5 \\
\hline$(1,5)$ & 0.017 & 95.4 & 0.026 & 73.1 & 0.042 & 23.9 \\
\hline$(2,5)$ & 0.016 & -133.1 & 0.008 & 179.5 & 0.098 & 32.2 \\
\hline$(3,5)$ & 0.023 & -177.4 & 0.034 & 180.0 & 0.114 & 40.3 \\
\hline$(4,5)$ & 0.018 & 115.1 & 0.041 & 150.6 & 0.104 & 41.5 \\
\hline$(5,5)$ & 0.026 & 68.7 & 0.017 & 113.6 & 0.099 & 37.5 \\
\hline$(9.5)$ & 0.027 & 134.6 & 0.011 & 61.8 & 0.044 & 41.3 \\
\hline$(10,5)$ & 0.008 & 133.7 & 0.014 & 11.9 & 0.019 & -0.8 \\
\hline$(11,5)$ & 0.007 & 16.4 & 0.005 & -115.9 & 0.029 & -131.3 \\
\hline$(12,5)$ & 0.012 & 43.8 & 0.017 & -148.7 & 0.018 & 148.5 \\
\hline$(13,5)$ & 0.006 & 62.8 & 0.012 & -145.9 & 0.045 & 66.7 \\
\hline
\end{tabular}




\begin{tabular}{|c|c|c|c|c|c|c|}
\hline$(1,6)$ & 0.022 & -14.8 & 0.020 & 0.1 & 0.061 & -92.5 \\
\hline$(2,6)$ & 0.011 & 119.4 & 0.012 & 63.0 & 0.103 & -76.1 \\
\hline$(3,6)$ & 0.021 & 102.1 & 0.046 & 102.5 & 0.126 & -70.9 \\
\hline$(4,6)$ & 0.020 & 29.1 & 0.056 & 78.4 & 0.112 & -76.4 \\
\hline$(5,6)$ & 0.027 & -38.0 & 0.030 & 29.2 & 0.094 & -67.2 \\
\hline$(9,6)$ & 0.016 & 80.7 & 0.014 & -9.6 & 0.016 & -51.7 \\
\hline$(10,6)$ & 0.008 & -125.3 & 0.020 & -45.3 & 0.009 & -129.5 \\
\hline$(11,6)$ & 0.011 & -127.4 & 0.008 & 35.2 & 0.037 & 180.0 \\
\hline$(12,6)$ & 0.004 & 59.7 & 0.011 & 150.0 & 0.013 & 120.8 \\
\hline$(13,6)$ & 0.007 & 47.0 & 0.015 & -165.8 & 0.036 & 3.5 \\
\hline$(1,7)$ & 0.019 & -131.2 & 0.008 & -79.9 & 0.054 & 163.5 \\
\hline$(2,7)$ & 0.013 & 128.5 & 0.014 & -71.5 & 0.078 & -179.0 \\
\hline$(3,7)$ & 0.012 & 30.1 & 0.027 & 3.0 & 0.110 & 178.0 \\
\hline$(4,7)$ & 0.026 & -51.7 & 0.043 & -13.1 & 0.123 & 175.0 \\
\hline$(5,7)$ & 0.028 & -89.0 & 0.038 & -57.2 & 0.098 & 176.0 \\
\hline$(9,7)$ & 0.021 & -29.5 & 0.020 & -96.1 & 0.018 & -102.8 \\
\hline$(10,7)$ & 0.012 & -115.4 & 0.023 & -121.4 & 0.005 & -34.0 \\
\hline$(11,7)$ & 0.016 & -166.2 & 0.013 & 94.2 & 0.024 & 116.0 \\
\hline$(12,7)$ & 0.006 & 170.5 & 0.020 & 61.3 & 0.010 & -53.9 \\
\hline$(13,7)$ & 0.004 & 56.4 & 0.003 & -139.3 & 0.054 & -64.0 \\
\hline
\end{tabular}




\begin{tabular}{|c|c|c|c|c|c|c|}
\hline$(1,8)$ & 0.025 & 130.4 & 0.021 & 158.6 & 0.077 & 70.3 \\
\hline$(2,8)$ & 0.018 & 60.4 & 0.028 & -163.7 & 0.109 & 71.7 \\
\hline$(3,8)$ & 0.020 & -21.5 & 0.032 & -118.1 & 0.130 & 82.4 \\
\hline$(4,8)$ & 0.021 & -58.6 & 0.037 & -116.7 & 0.131 & 89.4 \\
\hline$(5,8)$ & 0.012 & 140.3 & 0.043 & -146.5 & 0.092 & 80.9 \\
\hline$(9,8)$ & 0.020 & -103.0 & 0.020 & -134.3 & 0.015 & -22.6 \\
\hline$(10,8)$ & 0.017 & $160: 9$ & 0.028 & -166.6 & 0.025 & 8.6 \\
\hline$(11,8)$ & 0.021 & 132.7 & 0.015 & 94.9 & 0.034 & 56.5 \\
\hline$(12,8)$ & 0.004 & 152.9 & 0.025 & 19.9 & 0.004 & 102.9 \\
\hline$(13,8)$ & 0.003 & -101.8 & 0.018 & -38.2 & 0.036 & -120.5 \\
\hline$(1,9)$ & 0.032 & 31.2 & 0.025 & 105.0 & 0.070 & -55.0 \\
\hline$(2,9)$ & 0.019 & 2.6 & 0.019 & 129.1 & 0.102 & -47.5 \\
\hline$(3,9)$ & 0.010 & -66.9 & 0.020 & 172.5 & 0.119 & -18.8 \\
\hline$(4,9)$ & 0.002 & -115.3 & 0.038 & 146.0 & 0.113 & $-13 \cdot 0$ \\
\hline$(5,9)$ & 0.020 & 62.6 & 0.059 & 142.8 & 0.102 & -29.9 \\
\hline$(9,9)$ & 0.026 & -158.9 & 0.018 & 137.8 & 0.017 & -152.9 \\
\hline$(10,9)$ & 0.025 & 108.5 & 0.024 & 126.8 & 0.017 & -112.8 \\
\hline$(11,9)$ & 0.025 & 78.5 & 0.013 & 52.6 & 0.003 & $-11 \cdot 3$ \\
\hline$(12,9)$ & 0.013 & -48.0 & 0.022 & -42.3 & 0.029 & 126.1 \\
\hline$(13,9)$ & 0.014 & -100.2 & 0.019 & -112.1 & 0.028 & 159.9 \\
\hline
\end{tabular}




$\begin{array}{rrrrrrr}(1,10) & 0.027 & -55.6 & 0.018 & 24.4 & 0.059 & -136.3 \\ (2,10) & 0.017 & -88.4 & 0.020 & 7.9 & 0.110 & -134.8 \\ (3,10) & 0.004 & 145.0 & 0.018 & 31.5 & 0.117 & -122.7 \\ (4,10) & 0.009 & -158.2 & 0.040 & 59.1 & 0.105 & -118.5 \\ (5,10) & 0.029 & -60.9 & 0.051 & 63.4 & 0.097 & -133.5 \\ & & & & & & \\ (9,10) & 0.016 & 139.0 & 0.034 & 102.2 & 0.023 & 171.3 \\ (10,10) & 0.017 & 21.2 & 0.023 & 65.1 & 0.027 & -138.4 \\ (11,10) & 0.019 & -26.3 & 0.022 & -53.7 & 0.024 & -89.6 \\ (12,10) & 0.013 & -96.2 & 0.018 & -122.3 & 0.011 & 7.4 \\ (13,10) & 0.006 & -145.3 & 0.021 & -179.0 & 0.024 & 88.0\end{array}$

In addition, at $x=D_{c}=3.048 \mathrm{~m}$ and $y=h_{m}=1.448 \mathrm{~m}$ (door aperture),

$$
V_{z}=0.119 v \text { and } \psi_{z}=-40.2 \mathrm{deg} \text {. }
$$

A few important conclusions may be drawn from the above table.

(1) On the average, $v_{z}$ is the dominant component as it is expected.

(2) The values of $V_{z}$ are consisitently larger at $(i, j)$ with $i=3,4$ and 5 for all j's, since these positions are in the lit region directly illuminated by the source. The values of $v_{z}$ are relatively weaker at $(i, j)$ for $i=9$ through 13 near plane 5 , since those positions are in the shadow region.

(3) For $i=3,4$, and 5 (1it region), values of $V_{z}$ vary between a high of 0.146 volt and a low of 0.088 volt, indicating a standing-wave type of field distribution inside the building.

(4) Phases of the component fields change rather irregularly. 
In view of the subject presented in Section 3, one particular component field strength or polarization with respect to the narrow slot which may appear on an object to be placed inside the building may have more penetration effect than the other components or polarizations. For this reason, measurements of the component field strengths rather than the total field amplitude $\left(\left|E_{x}\right|^{2}+\left|E_{y}\right|^{2}+\left|E_{z}\right|^{2}\right)^{1 / 2}$ should prove more meaningful.

A second set of measured data with a new measuring antenna height $\left(h_{m}=1.905 \mathrm{~m}\right)$ is tabulated as follows:

Table 5

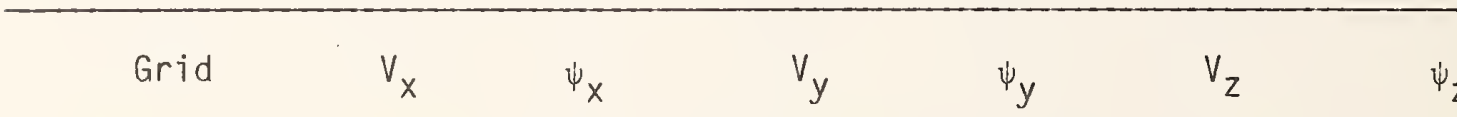

Position in volts in deg. in volts in deg. in volts in deg.

$\begin{array}{rrrrrrr}(1,1) & 0.055 & 179.0 & 0.055 & -49.7 & 0.059 & 87.5 \\ (2,1) & 0.063 & -165.9 & 0.037 & 176.3 & 0.113 & 115.6 \\ (3,1) & 0.015 & 171.6 & 0.074 & 176.3 & 0.157 & 111.9 \\ (4,1) & 0.028 & 63.1 & 0.034 & -154.6 & 0.153 & 101.0 \\ (5,1) & 0.030 & 91.4 & 0.046 & -61.2 & 0.119 & 121.4 \\ (9,1) & 0.025 & 146.3 & 0.008 & -8.5 & 0.022 & -54.0 \\ (10,1) & 0.020 & 143.8 & 0.012 & -18.8 & 0.016 & -152.3 \\ (11,1) & 0.014 & 139.6 & 0.017 & -99.3 & 0.026 & 66.5 \\ (12,1) & 0.010 & 114.5 & 0.009 & 175.6 & 0.017 & 26.9 \\ (13,1) & 0.008 & 62.9 & 0.020 & 95.2 & 0.020 & -137.1\end{array}$




\begin{tabular}{|c|c|c|c|c|c|c|}
\hline$(1,2)$ & 0.037 & 99.3 & 0.042 & -117.1 & 0.060 & -22.5 \\
\hline$(2,2)$ & 0.063 & 118.6 & 0.034 & 124.1 & 0.083 & -8.2 \\
\hline$(3,2)$ & 0.030 & 98.7 & 0.064 & 114.5 & $0.14,0$ & -3.6 \\
\hline$(4,2)$ & 0.017 & -9.2 & 0.024 & 156.8 & 0.152 & -5.3 \\
\hline$(5,2)$ & 0.012 & -73.3 & 0.019 & -138.2 & 0.124 & 2.2 \\
\hline$(9,2)$ & 0.022 & 43.1 & 0.003 & $-41 \cdot 5$ & 0.027 & -137.8 \\
\hline$(10,2)$ & 0.013 & 52.0 & 0.007 & -18.7 & 0.035 & 143.3 \\
\hline$(11.2)$ & 0.009 & 155.6 & 0.010 & -114.1 & 0.037 & 54.7 \\
\hline$(12,2)$ & 0.012 & 179.0 & 0.003 & 132.4 & 0.011 & 5.7 \\
\hline$(13,2)$ & 0.006 & 75.1 & 0.017 & 61.7 & 0.031 & 169.0 \\
\hline$(1,3)$ & 0.030 & -17.5 & 0.044 & 170.5 & 0.068 & -129.6 \\
\hline$(2,3)$ & 0.037 & 22.1 & 0.029 & 60.7 & 0.096 & $-124 \cdot 6$ \\
\hline$(3,3)$ & 0.030 & -19.4 & 0.046 & 21.5 & 0.140 & -106.9 \\
\hline$(4,3)$ & 0.045 & -79.7 & 0.025 & -8.3 & 0.154 & $-100 \cdot 2$ \\
\hline$(5,3)$ & 0.026 & -98.3 & 0.010 & 46.0 & 0.122 & -114.5 \\
\hline$(9,3)$ & 0.038 & -18.3 & 0.006 & 179.8 & 0.048 & -142.7 \\
\hline$(10,3)$ & 0.028 & -21.4 & 0.008 & 162.6 & 0.030 & 125.4 \\
\hline$(11,3)$ & 0.011 & -58.8 & 0.015 & -173.0 & 0.041 & -19.7 \\
\hline$(12,3)$ & 0.011 & -128.1 & 0.008 & 80.1 & 0.031 & -79.1 \\
\hline$(13,3)$ & 0.008 & -79.1 & 0.024 & 34.7 & 0.032 & 177 \\
\hline
\end{tabular}




$\begin{array}{lrrrrrr}(1,4) & 0.020 & -132.7 & 0.044 & 133.0 & 0.071 & 134.4 \\ (2,4) & 0.035 & -64.5 & 0.012 & -43.3 & 0.123 & 138.7 \\ (3,4) & 0.038 & -94.9 & 0.052 & -67.0 & 0.140 & 155.3 \\ (4,4) & 0.035 & -164.3 & 0.034 & -89.0 & 0.132 & 166.5 \\ (5,4) & 0.032 & 154.4 & 0.008 & -6.0 & 0.123 & 159.1 \\ (9,4) & 0.011 & -92.3 & 0.012 & 128.9 & 0.039 & 116.9 \\ (10,4) & 0.008 & -13.9 & 0.014 & 157.3 & 0.028 & 39.5 \\ (11,4) & 0.008 & 53.2 & 0.022 & -152.7 & 0.050 & -52.9 \\ (12,4) & 0.007 & 86.4 & 0.005 & -90.1 & 0.024 & -103.5 \\ (13,4) & 0.003 & -79.4 & 0.023 & 15.8 & 0.036 & 128.5\end{array}$

$\begin{array}{rrrrccr}(1,5) & 0.021 & 120.2 & 0.027 & 96.5 & 0.064 & 24.2 \\ (2,5) & 0.011 & -135.5 & 0.015 & 179.5 & 0.110 & 35.5 \\ (3,5) & 0.025 & -178.3 & 0.040 & -162.0 & 0.127 & 49.4 \\ (4,5) & 0.046 & 128.8 & 0.038 & 171.1 & 0.123 & 50.5 \\ (5,5) & 0.040 & 107.6 & 0.019 & 134.2 & 0.123 & 50.4\end{array}$

$\begin{array}{rrrrrrr}(9,5) & 0.033 & 162.9 & 0.022 & 72.0 & 0.050 & 56.5 \\ (10,5) & 0.016 & 148.9 & 0.010 & 56.0 & 0.020 & 11.6 \\ (11,5) & 0.012 & 100.3 & 0.018 & -131.6 & 0.035 & -118.3 \\ (12,5) & 0.020 & 86.1 & 0.017 & -101.9 & 0.025 & 164.1 \\ (13,5) & 0.009 & 80.4 & 0.011 & -31.2 & 0.054 & 87.1\end{array}$




\begin{tabular}{|c|c|c|c|c|c|c|}
\hline$(1,6)$ & 0.017 & 13.1 & 0.022 & 37.2 & 0.087 & -75.1 \\
\hline$(2,6)$ & 0.010 & 95.8 & 0.020 & 103.5 & 0.118 & -70.6 \\
\hline$(3,6)$ & 0.029 & 82.4 & 0.047 & 116.6 & 0.130 & -51.4 \\
\hline$(4,6)$ & 0.029 & 42.0 & 0.048 & 98.6 & 0.120 & -63.1 \\
\hline$(5,6)$ & 0.031 & -11.2 & 0.028 & 50.5 & 0.107 & -59.5 \\
\hline$(9,6)$ & 0.016 & 110.6 & 0.024 & 29.4 & 0.020 & -53.9 \\
\hline$(10,6)$ & 0.013 & -113.6 & 0.012 & 3.5 & 0.015 & -127.9 \\
\hline$(11,6)$ & 0.011 & -142.8 & 0.009 & 152.4 & 0.043 & -170.3 \\
\hline$(12,6)$ & 0.014 & 100.4 & 0.007 & -142.9 & 0.015 & 130.1 \\
\hline$(13,6)$ & 0.008 & 91.7 & 0.015 & -89.4 & 0.046 & 17.8 \\
\hline$(1,7)$ & 0.020 & -109.8 & 0.013 & -44.7 & 0.078 & 178.1 \\
\hline$(2,7)$ & 0.011 & 173.3 & 0.014 & -14.9 & 0.098 & 176.1 \\
\hline$(3,7)$ & 0.010 & 42.7 & 0.034 & 32.5 & 0.122 & -168.5 \\
\hline$(4,7)$ & 0.027 & -39.8 & 0.041 & 3.9 & 0.130 & -167.9 \\
\hline$(5,7)$ & 0.033 & -75.1 & 0.042 & -47.9 & 0.111 & -171.4 \\
\hline$(9,7)$ & 0.020 & -18.6 & 0.024 & -70.5 & 0.019 & -91.0 \\
\hline$(10,7)$ & 0.021 & -100.2 & 0.013 & $-128 . ?$ & 0.003 & 2.1 \\
\hline$(11,7)$ & 0.019 & -127.4 & 0.023 & 120.3 & 0.027 & 118.0 \\
\hline$(12,7)$ & 0.002 & 163.4 & 0.015 & 90.9 & 0.018 & -29.4 \\
\hline$(13,7)$ & 0.008 & 46.5 & 0.011 & -71.0 & 0.055 & -53.0 \\
\hline
\end{tabular}




$\begin{array}{rrrrrrr}(1,8) & 0.030 & 152.6 & 0.018 & -149.6 & 0.083 & 86.0 \\ (2,8) & 0.015 & 146.7 & 0.021 & -127.2 & 0.109 & 84.7 \\ (3,8) & 0.014 & -84.4 & 0.028 & -79.8 & 0.127 & 97.1 \\ (4,8) & 0.014 & -136.6 & 0.038 & -86.7 & 0.121 & 101.9 \\ (5,8) & 0.024 & 154.0 & 0.049 & -115.5 & 0.093 & 92.1 \\ & & & & & & \\ (9,8) & 0.019 & -101.6 & 0.034 & -109.3 & 0.018 & -1.2 \\ (10,8) & 0.013 & 172.6 & 0.022 & -158.4 & 0.027 & 19.5 \\ (11,8) & 0.013 & 134.2 & 0.028 & 93.4 & 0.032 & 63.9 \\ (12,8) & 0.008 & -10.5 & 0.025 & 45.8 & 0.003 & -178.8 \\ (13,8) & 0.007 & -32.5 & 0.019 & -61.7 & 0.038 & -111.6\end{array}$

$\begin{array}{rrrrrrr}(1,9) & 0.024 & 55.3 & 0.014 & 162.2 & 0.083 & -33.5 \\ (2,9) & 0.016 & -2.6 & 0.017 & -177.7 & 0.112 & -31.3 \\ (3,9) & 0.009 & -58.2 & 0.023 & -170.5 & 0.118 & -15.3 \\ (4,9) & 0.005 & 141.9 & 0.032 & 172.2 & 0.109 & -5.9 \\ (5,9) & 0.028 & 82.7 & 0.041 & 161.8 & 0.100 & -23.4\end{array}$

$\begin{array}{rrrrrrr}(9,9) & 0.019 & -153.1 & 0.017 & -178.4 & 0.016 & -135.9 \\ (10,9) & 0.028 & 98.8 & 0.013 & 125.2 & 0.018 & -106.3 \\ (11,9) & 0.017 & 64.6 & 0.021 & 36.5 & 0.002 & 1.4 \\ (12,9) & 0.017 & -77.2 & 0.018 & -8.1 & 0.028 & 144.8 \\ (13,9) & 0.015 & -111.1 & 0.013 & -114.4 & 0.036 & 164.0\end{array}$




$\begin{array}{rrrrrrr}(1,10) & 0.025 & -55.6 & 0.010 & 23.6 & 0.077 & -126.4 \\ (2,10) & 0.015 & -67.2 & 0.007 & 3.8 & 0.111 & -127.5 \\ (3,10) & 0.004 & 41.8 & 0.012 & 108.5 & 0.107 & -117.1 \\ (4,10) & 0.006 & -16.5 & 0.033 & 103.5 & 0.079 & -114.6 \\ (5,10) & 0.029 & -30.1 & 0.041 & 86.0 & 0.087 & -121.9 \\ & & & & & & \\ (9,10) & 0.011 & 107.7 & 0.035 & 118.0 & 0.030 & 178.3 \\ (10,10) & 0.021 & 45.5 & 0.015 & 71.5 & 0.028 & -129.1 \\ (11,10) & 0.012 & -15.8 & 0.022 & -44.6 & 0.025 & -69.1 \\ (12,10) & 0.020 & -120.5 & 0.009 & -102.3 & 0.011 & 37.6 \\ (13,10) & 0.011 & -164.6 & 0.021 & 163.7 & 0.028 & 102.3\end{array}$

Note that the general conclusions made for the data presented in Table 4 with $h_{m}=1.448 \mathrm{~m}$. hold good also for the data presented in Table 5 with $h_{m}=$ $1.905 \mathrm{~m}$. The highest value for $V_{z}$ is now 0.157 volt (against 0.146 volt for Table 4) at position $(3,1)$. This result is expected from the behavior of the ground reflection coefficient $R_{v}$ given in (92). Thus, if possible, measurements should be made with the feed point of the measuring antennas farther away from the floor. Of course, this is limited by the supporting pole and the convenience of making measurements. 
Based on the measured data presented in Tables 4 and 5, and the hybrid approach represented by (77) to (79), we obtain the following results. (1) When the number of patches to be used is eight on Planes 1, 2, and 3 represented by $m^{(1)}=n^{(1)}=m^{(2)}=n^{(2)}=m^{(3)}=n^{(3)}=2$, the minimum number of locations, $p$, where the measured data are needed is also eight in accordance with (98) or (99). Thus, we choose the measurement positions $(1,1),(2,1),(1,2)$ and $(2,2)$ with two measuring antenna heights so that the equal sign in (98) or (99) holds. The specific results are:

for Plane 1 (floor) where $z^{\prime}=0$,

$\begin{array}{lll}\text { position }(1,1) & J_{x}=0.5750+j 0.3598 & J_{y}=-0.4200-j 0.4349 \\ \text { position }(2,1) & J_{x}=0.4628+j 1.0371 & J_{y}=-0.3880+j 0.3937 \\ \text { position }(1,2) & J_{x}=0.2582-j 0.3361 & J_{y}=-0.2460-j 0.5865 \\ \text { position }(2,2) & J_{x}=-0.9534-j 0.5706 & J_{y}=0.7870+j 0.2328,\end{array}$

for Plane 2 (front) where $x^{\prime}=0$,

$\begin{array}{lll}\text { position }(1,1) & J_{y}=1.3713-j 0.4874 & J_{z}=-0.9528+j 0.2119 \\ \text { position }(2,1) & J_{y}=-0.1948+j 0.1866 & J_{z}=-0.6499-j 0.0266 \\ \text { position }(1,2) & J_{y}=-0.9331+j 0.1377 & J_{z}=0.1453+j 0.8761 \\ \text { position }(2,2) & J_{y}=0.1786+j 0.0755 & J_{z}=-0.2295-j 0.8728,\end{array}$
and for Plane 3 (right) where $y^{\prime}=0$,

\begin{tabular}{|c|c|c|c|c|c|c|}
\hline position $(1,1)$ & $J_{x}=-2.1408$ & $+\quad j 0.0044$ & $\mathrm{~J}_{\mathrm{z}}=$ & -1.2517 & . & j0.3303 \\
\hline position $(2,1)$ & $J_{x}=-0.1024$ & $-j 0.1476$ & $\mathrm{~J}_{z}=$ & -0.9308 & & j0.3842 \\
\hline position $(1,2)$ & 1.5798 & $+\quad j 0.2727$ & $\mathrm{~J}_{\mathrm{z}}=$ & 0.0751 & - & $j 1.2033$ \\
\hline position $(2,2)$ & $J_{x}=0.0568$ & - j0.1989 & $J_{x}$ & 0.0481 & & j1.2338 \\
\hline
\end{tabular}

(2) When $m^{(1)}=n^{(1)}=m^{(2)}=m^{(3)}=3$ and $n^{(2)}=n^{(3)}=2$, we require $p \geqslant 14$. Actually we choose the measurement positions $(1,1),(2,1),(3,1),(1,2),(2,2)$, 
$(3,2),(1,3),(2,3)$, and $(3,3)$, with two measuring antenna heights, a total of 18 positions. Thus, the greater sign in (99) holds. The least-square-fit results are: for Plane 1 where $z^{\prime}=0$,

$\begin{array}{lll}\text { position }(1,1) & J_{x}=0.0496+j 0.0705 & J_{y}=0.0705-j 0.0432 \\ \text { position }(2,1) & J_{x}=-0.0494-j 0.1247 & J_{y}=0.1369+j 0.0110 \\ \text { position }(3,1) & J_{x}=0.0274+j 0.0885 & J_{y}=-0.0898-j 0.0077 \\ \text { position }(1,2) & J_{x}=-0.1276-j 0.0836 & J_{y}=-0.0908+j 0.0498 \\ \text { position }(2,2) & J_{x}=0.1340+j 0.1464 & J_{y}=-0.1433-j 0.0202 \\ \text { position }(3,2) & J_{x}=-0.0712-j 0.1124 & J_{y}=0.0780+j 0.0154 \\ \text { position }(1,3) & J_{x}=0.0627+j 0.0519 & J_{y}=-0.0725-j 0.0610 \\ \text { position }(2,3) & J_{x}=-0.0536-j 0.0808 & J_{y}=0.0972+j 0.0545 \\ \text { position }(3,3) & J_{x}=0.0201+j 0.0589 & J_{y}=-0.0353-j 0.0331,\end{array}$

for Plane 2 where $x^{\prime}=0$,

$\begin{array}{lll}\text { position }(1,1) & J_{y}=0.0350-j 0.0439 & J_{z}=0.0174-j 0.0146 \\ \text { position }(2,1) & J_{y}=-0.0316+j 0.0370 & J_{z}=0.0364-j 0.0289 \\ \text { position }(3,1) & J_{y}=0.0447-j 0.0075 & J_{z}=0.0275-j 0.0140 \\ \text { position }(1,2) & J_{y}=-0.0170+j 0.0185 & J_{z}=0.0033-j 0.0340 \\ \text { position }(2,2) & J_{y}=0.0137-j 0.0129 & J_{z}=0.0339+j 0.0263 \\ \text { position }(3,2) & J_{y}=-0.0270+j 0.0049 & J_{z}=-0.0204-j 0.0117,\end{array}$

and for Plane 3 where $y^{\prime}=0$,

$\begin{array}{lll}\text { position }(1,1) & J_{x}=0.0505-j 0.0057 & J_{z}=0.0254+j 0.0067 \\ \text { position }(2,1) & J_{x}=-0.0710+j 0.0250 & J_{z}=0.0001-j 0.0125 \\ \text { position }(3,1) & J_{x}=0.0321-j 0.0213 & J_{z}=-0.0046+j 0.0012 \\ \text { position }(1,2) & J_{x}=-0.0446-j 0.0084 & J_{z}=-0.0581+j 0.0352 \\ \text { position }(2,2) & J_{x}=0.0638-j 0.0048 & J_{z}=0.0138-j 0.0600 \\ \text { position }(3,2) & J_{x}=-0.0298+j 0.0080 & J_{z}=-0.0143+j 0.0315 .\end{array}$


(3) When we use $m^{(1)}=n^{(1)}=m^{(2)}=m^{(3)}=4$ and $n^{(2)}=n^{(3)}=2$, we require $p \geqslant 16$. Actually we choose the measurements positions, $(1,1),(2,1),(3,1)$, $(4,1),(1,2),(2,2),(3,2),(4,2),(1,3),(2,3),(3,3),(4,3),(1,4),(2,4)$, $(3,4)$, and $(4,4)$ with two measuring antenna heights, a total of 32 positions. Thus, the greater sign in (99) also holds here. The specific results are: (also in the sense of least square-fit)

for Plane 1 where $z^{\prime}=0$,

$\begin{array}{lll}\text { position }(1,1) & J_{x}=-0.0642-j 0.0120 & J_{y}=0.0770+j 0.0337 \\ \text { position }(2,1) & J_{x}=0.1257-j 0.0152 & J_{y}=-0.1334+j 0.0348 \\ \text { position }(3,1) & J_{x}=-0.0948+j 0.0174 & J_{y}=0.1441-j 0.0577 \\ \text { position }(4,1) & J_{x}=0.0456+j 0.0060 & J_{y}=-0.0852+j 0.0102 \\ \text { position }(1,2) & J_{x}=0.1129-j 0.0859 & J_{y}=-0.0985-j 0.0197 \\ \text { position }(2,2) & J_{x}=-0.1797+j 0.1288 & J_{y}=0.1543-j 0.0446 \\ \text { position }(3,2) & J_{x}=0.1447-j 0.0659 & J_{y}=-0.1803+j 0.0552 \\ \text { position }(4,2) & J_{x}=-0.0770-j 0.0015 & J_{y}=0.1098+j 0.0063 \\ \text { position }(1,3) & J_{x}=-0.1266+j 0.1000 & J_{y}=0.0750-j 0.0255 \\ \text { position }(2,3) & J_{x}=0.1949-j 0.1147 & J_{y}=-0.1379+j 0.0678 \\ \text { position }(3,3) & J_{x}=-0.1585+j 0.0349 & J_{y}=0.1776-j 0.0469 \\ \text { position }(4,3) & J_{x}=0.0717+j 0.0195 & J_{y}=-0.1044-j 0.0243 \\ \text { position }(1,4) & J_{x}=0.0913-j 0.0314 & J_{y}=-0.0681+j 0.0279 \\ \text { position }(2,4) & J_{x}=-0.1295+j 0.0254 & J_{y}=0.1222-j 0.0554 \\ \text { position }(3,4) & J_{x}=0.0932+j 0.0184 & J_{y}=-0.1312+j 0.0240 \\ \text { position }(4,4) & J_{x}=-0.0277-j 0.0291 & J_{y}=0.0611+j 0.0242,\end{array}$


for Plane 2 where $x^{\prime}=0$,

$\begin{array}{lll}\text { position }(1,1) & J_{y}=0.0143-j 0.0057 & J_{z}=-0.0125-j 0.0083 \\ \text { position }(2,1) & J_{y}=-0.0419-j 0.0099 & J_{z}=-0.0105-j 0.0079 \\ \text { position }(3,1) & J_{y}=0.0522+j 0.0234 & J_{z}=0.0185+j 0.0149 \\ \text { position }(4,1) & J_{y}=-0.0159-j 0.0256 & J_{z}=-0.0123-j 0.0180 \\ \text { position }(1,2) & J_{y}=-0.0009-j 0.0027 & J_{z}=0.0548+j 0.0570 \\ \text { position }(2,2) & J_{y}=0.0091+j 0.0144 & J_{z}=-0.0625-j 0.0301 \\ \text { position }(3,2) & J_{y}=-0.0182-j 0.0143 & J_{z}=0.0282+j 0.0179 \\ \text { position }(4,2) & J_{y}=0.0062+j 0.0136 & J_{z}=-0.0004-j 0.0042,\end{array}$

and for Plane 3 where $y^{\prime}=0$

$\begin{array}{lll}\text { position }(1,1) & J_{x}=0.0110-j 0.0056 & J_{z}=0.0650-j 0.0023 \\ \text { position }(2,1) & J_{x}=0.0116+j 0.0274 & J_{z}=-0.0477+j 0.0469 \\ \text { position }(3,1) & J_{x}=-0.0410-j 0.0080 & J_{z}=0.0291-j 0.0498 \\ \text { position }(4,1) & J_{x}=0.0218-j 0.0133 & J_{z}=0.0016+j 0.0209 \\ \text { position }(1,2) & J_{x}=-0.0141-j 0.0102 & J_{z}=-0.0973-j 0.0757 \\ \text { position }(2,2) & J_{x}=0.0085-j 0.0141 & J_{z}=0.1167+j 0.0304 \\ \text { position }(3,2) & J_{x}=0.0148+j 0.0110 & J_{z}=-0.0770-j 0.0226 \\ \text { position }(4,2) & J_{x}=-0.0083+j 0.0057 & J_{z}=0.0036+j 0.0169 .\end{array}$

Results for other cases with $m(1)=n^{(1)}=m^{(2)}=m^{(3)}=5, n^{(2)}=n(3)=2$ and $m^{(1)}=n^{(1)}=m^{(2)}=m^{(3)}=6, n^{(2)}=n^{(3)}=2$ are also available but not listed here.

Comparing the results in (100) with those corresponding ones in (103) and (106), we find that $J_{x}$ and $J_{y}$ in Plane 1 vary substantially without clear indications of convergence to certain values when the total number of patches used is increased up to $4 \times 4 \times 2$. This is not unexpected because the incremental 
interval of $\Delta x=\Delta y=0.6096 \mathrm{~m}$ (approximately $0.3 \lambda$ ) is too large. Should a smaller increment in the order of $\Delta x=\Delta y=0.2 \mathrm{~m}$ (or $0.1 \lambda$ ) be used, a tendency of convergence for $J_{x}$ and $J_{y}$ may be established. Same comments also apply to $J_{y}$ and $J_{z}$ in Plane 2 and to $J_{x}$ and $J_{z}$ in Plane 3.

When the equivalent surface current densities obtained in (100) through (108), however imperfect as they are, are used in (77) to (79) for computing the field strengths at spaces inside the building, the results are still quite interesting as seen from the summary presented below.

With the current densities in (100) to (102), based on eight patches $(2 \times 2 \times$ 2), substituted in (77) to (79), we obtain the field strength components at $x=$ $0.9144 \mathrm{~m}\left(\mathrm{x}_{0}\right), y=1.0668 \mathrm{~m}\left(\mathrm{y}_{0}\right)$, and $z=1.4478 \mathrm{~m}\left(\mathrm{~h}_{\mathrm{m}}\right)$ as follows:

$$
E_{x}=0.07141-171.4^{\circ}, E_{y}=0.05361-46.3^{\circ}, E_{z}=0.0536 / 107.2^{\circ} \mathrm{V} / \mathrm{m} .
$$

Since the effective length of the dipoles being used in the measurement is approximately $l_{x}=l_{y}=l_{z}=0.5527 \mathrm{~m}$, the received voltage components becomes

$$
v_{x}=0.03951-171.4^{\circ}, v_{y}=0.02961-46.3^{\circ}, v_{z}=0.02961107 .2^{\circ} \mathrm{v} \text {, }
$$

which are, of course, the same as those appearing at position $(1,1)$ in Table 1 because position $(1,1)$ is one of the data points. Similarly, the same results at positions $(1,2),(2,1)$ and $(2,2)$ in Table 1 and those in Table 2 with $x=0.9144 \mathrm{~m}$, $y=1.0668 \mathrm{~m}$ and $z=1.9050 \mathrm{~m}$ (a second measurement height) are also recovered.

The voltage components computed at $x=1.2192 \mathrm{~m}, y=1.3716 \mathrm{~m}$ and $z=1.6764 \mathrm{~m}$, which are not one of the original data points, with different number of patches are: (1) With $2 \times 2 \times 2$ or the results in (100) through (102), $v_{x}=1.59581160 .0^{\circ}, v_{y}=1.07801-14.8^{\circ}, v_{z}=0.05841129 .7^{\circ} \mathrm{v} ;$

(2) With $3 \times 3 \times 2$ or the results in (103) through (105), $v_{x}=0.0663 / 162,7^{\circ}, v_{y}=0.0037127 .5^{\circ}, v_{z}=0.0781 / 86.2^{\circ} v ;$ 
(3) With $4 \times 4 \times 2$ or the results in (106) through (108), $v_{x}=0.05311-175.1^{\circ}, V_{y}=0.0246178 .9^{\circ}, \quad V_{z}=0.0776183 .7^{\circ} \mathrm{v}$.

For other different numbers of patches such as $5 \times 5 \times 2,6 \times 6 \times 2$ and $7 \times 6 \times 2$ of which the current densities are also available though not explicitly listed, the results of voltage components are also presented below for the analysis purpose.

\begin{tabular}{llccccc}
\hline $\begin{array}{c}\text { Number of } \\
\text { Patches }\end{array}$ & $\begin{array}{c}v_{x} \\
\text { in volts }\end{array}$ & $\begin{array}{c}\psi_{x} \\
\text { in deg. }\end{array}$ & $\begin{array}{c}v_{y} \\
\text { in volts }\end{array}$ & $\begin{array}{c}\psi_{y} \\
\text { in deg. }\end{array}$ & $\begin{array}{c}v_{z} \\
\text { in volts }\end{array}$ & $\begin{array}{c}\psi_{z} \\
\text { in deg. }\end{array}$ \\
\hline $5 \times 5 \times 2$ & 0.0433 & 155.8 & 0.0245 & 125.6 & 0.0811 & 81.1 \\
$6 \times 6 \times 2$ & 0.0539 & 173.2 & 0.0124 & -66.7 & 0.0828 & 90.3 \\
$7 \times 6 \times 2$ & 0.0407 & 165.1 & 0.0017 & 31.2 & 0.0807 & 84.7 \\
\hline
\end{tabular}

Clearly, the voltage components or their field strengths counter parts do converge to reasonable levels when the number of patches used in the computation is increased. Furthermore, the $z$ component is still the dominant one as it should be even with the number of patches as small as $3 \times 3 \times 2$. The $y$-component is the weakest as it should be, since the direction of propagation is along the $y$-axis. This simple analysis indicates the method proposed in this report for computing the field strength inside a building based on the limited number of measurement data may be satisfactory to a certain degree. With large computing facilities available to accommodate a larger number of patches, the accuracy in computation and the ability to predict the field strength distribution inside a building similar to the geometry shown in figure 9 can be improved. 


\section{CONCLUDING REMARKS}

The problem of determining the field distribution inside an empty building or cavity with apertures due to an external source has been formulated for two different configurations. One of them requires that the aperture take the form of a horizontal narrow slot contained in an infinite conducting plane, that the cavity wall is lossy, that the cavity is in free space, and that the wave from the external source is normally incident on the slot. For this case, the problem is solved by an application of the equivalence principle and the generalized network presentation. It is found that at natural resonances the field amplitude in the slot aperture is inversely proportional to the square-root of the wall conductivity and that a field of high strength can exist inside the cavity.

The other configuration is large buildings and apertures situated on a lossy but homogeneous ground. For this latter case, the field distribution inside the building is determined by an analytical means together with a limited number of measurement data. This approach enables an engineer to make a quick on-site check with a moderate computing facility available, even though the accuracy is not very good. To improve the accuracy in the final computed results, a larger computing facility will be required.

\section{ACKNOWLEDGMENTS}

The authors are thankful to M. Kanda and G. Reeve for their suggestions and comments during the course of this study. Help received in computer programming from $P$. N. Swarztrauber is gratefully acknowledged. The work leading to the results reported herein was partly supported by U.S. Army Communications-Electronics Engineering Installation Agency. 


\section{REFERENCES}

[1] Mittra, R.; Ralmat-Samii, Y.; Jamnejad, D.V.; Davis, W.A. A new look at the thin plate scattering problem. Radio Sci. 8 (10): 869-875; 1973 October.

[2] Poggio, A.J.; Miller, E.K. Integral equation solutions for three-dimensional scattering problems, chapter 4 of Computer techniques for electromagnetics, R. Mittra, Ed. New York, NY: Pergamon Press; 1973.

[3] Mentzer, J.R. Scattering and diffraction of radio waves, New York, NY: Pergamon Press; 1955.

[4] Richmond, J.H. A wire-grid model for scattering by conducting bodies. IEEE Trans. on Antennas and Prop. AP-14(6): 782-786; 1966 November.

[5] Tsai, L.L.; Dudley, D.G.; Wilton, D.R. Electromagnetic scattering by a three-dimensional conducting rectangular box, J. of Applied Physics. 45(10): 4393-4400; 1974 October.

[6] Harrington, R.F. Field computation by moment methods. New York, NY: McMillan; 1968.

[7] Schelkunoff, S.A. Some equivalence theorems of electromagnetics and their application to radiation problems. Bell System Tech. J. 15: 92-112; 1936.

[8] Harrington, R.F.; Mautz, J.R. A generalized network formulation for aperture problems. IEEE Trans. on Antennas and Prop. AP-24 (6): 870-872;

1976 November.

[9] Harrington, R.F.; Mautz, J.R. Electromagnetic transmission through an aperture in a conducting plane. AEUं, 31: 81-87; 1977.

[9a] Cheng, D.K.; Liang, C.H. Electromagnetic fields in cavities with a slotaperture at natural resonance. IEEE International Symposium on Electromagnetic Compatibility: 489-494; 1981.

[10] Collin, R.E. Field theory of guided waves. New York, NY: Mc-Graw-Hill; 1960.

[11] Stewart, G.W. Introduction to matrix computations, chapter 5, esp. p. 214and 217 et seq. New York, NY: Academic Press; 1973.

[12] ibid., p. 108 .

[13] ibid.k p. 217 et seq.

[14] Dongarra, J.J.; Moler, C.B.; Bunch, J.R.; Steward, G.W. LINPACK user's guide. Philadelphia: SIAM Publications; 1979. pp 9.1.

[15] CQRDCT and CQRSLT were modified from CQRDC and CQRLS by Dr. PauT $N$. Swarztrauber, Scientific Computing Division, National Center for Atmospheric Research, Boulder, Colorado while on loan to the Scientific Computing Division, Center for Applied Mathematics, Nat. Bur. of Stand. (U.S.). Boulder, Colorado. 


\section{APPENDIX A. FORTRAN PROGRAMS}

\section{A.1 CURRENT Program}

PROGRAM CURRENT (INPUT, OUTPUT, TAPES = INPUT, TAPEG = OUTPUT, TAPET)

C THIS PROGRAM READS FIELD DATA IN VOLTS

C AND COMPUTES CURRENTS IN PLANES 1, 2, AND 3

COMPLEX OMS4P, SO4PE, GMNI, GNL2, GML3, S1, S2, S3, AMN1, ANL2, AML 3

1

, BMN 1, BNL2, BML 3, CMN 1, CNL2,CML3, CJX1, CJX3, CJYI, CJY2

2

, E (24), C J Z2, C JZ3, A, D IAG $(24), C 1, R$ SUBV, ESUBZ, XS (24)

INTEGER JPVT $(24), N X T O T H(2), N Y T O T H(2), \operatorname{IXH}(13,2), \operatorname{IYH}(16,2)$

COMMON A $(24,24)$

REAL MU,KAY, LSUBZ

NPRNTI $=0$

NPRNT $2=0$

NPRNT $3=0$

NPRNT $4=0$

NPRNT5 $=0$

C NPRNTI CONTROLS PRINTING DF OMEGA,MU,EPS,KAY, OMS4P, SOLPE

$C$ NPRNT2 CONTROLS PRINTINT OF XP, YP, ZP AND COEFFICIENTS

C NPRNT3 CONTROLS PRINTING OF MA,NA

C NPRNT4 CONTROLS PRINTING OF DIAGCNALS

C NPRNTS CONTROLS PRINTING OF RESIDUALS

MDIMA $=24$

NXTOTH $(1)=2$

NXTOTH(2) $=2$

NYTOTH $(1)=2$

NYTOTH $(2)=2$

$\operatorname{IXH}(1,1)=1$

IXH(1,2) $=1$

$\operatorname{IXH}(2,1)=2$

$\operatorname{IXH}(2,2)=2$

$\operatorname{IYH}(1,1)=1$

I $Y H(1,2)=1$

$\operatorname{I~} Y H(2,1)=2$

$\operatorname{IYH}(2,2)=2$

$D X=.6096$

$D Y=.6096$

$D Z \approx .4572$

$D X D Y=D X * D Y$

$D X D Z=D X * D Z$

$D Y D Z=D Y * D Z$

XSTART $=.9144$

YSTART $=1.0668$

ZSTART $=1.4478$

FREO $=1.481 E 8$

$P I=4 . * A T A N(1$.

OMEGA $=2$. *PI*FREO 
$M U=4 \cdot E-7 * P I$

$E P S=1 \cdot E-9 /(36 . \# P I)$

$L S=1$

$L F=2$

$K A Y=O M E G A * S Q R T$ (MU*EPS)

OMS4P =CMPLX $(0$, , OMEGA $\$ M U /(4$, \#I) )

SO4PE $=-C M P L X(0 ., .25 /(O M E G A \neq P I * E P S))$

IF (NPRNT1.GT.O) WRITE $(6,15)$ OMEGA,MU, EPS, KAY, OMS 4P, SO $4 P E$

15 FORMAT (

ILISTED BELOH\#/(BE15.6))

C

c

I $F=-2$

DO $100 \quad I Z=L S, L F$

$Z=(F L O A T(I Z)-1) * D Z+.Z S T A R T$

NXTOTENXTOTH(IZ)

NYTOT $=$ NYTOTH $(I Z)$

DO $100 \quad I R=I$, NYTOT

$I Y=I Y H(I R, I Z)$

$Y=(F L O A T(I Y)-1) \# D Y+.Y S T A R T$

65

DO $100 \quad I C=1, N X T O T$

$I X=I X H(I C, I Z)$

$x=(F L O A T(I X)-1 \cdot) \# D X+X S T A R T$

$I F=I F+3$

IF (NPRNT2.GT.0) HRITE $(6,1) \quad X, Y, Z$

1 FORMATI/1THE FOLLOWING COEFFICIENTS CORRESPOND TO THE FIEL IPOINT $* 1 * X=* F 8.4 *, Y=* F 8.4 *, Z=\$ F 8.4 / 1$

C COMPUTE COEFFICIENTS IN THE FLOOR OR $Z=0$ PLANE

C (SUBSCRIPT 1, BOTTOM XY-PLANE, FLOOR)

75

C

$J U=-1$

NYT $=$ NYTOTH(1)

DO 101 IN $=1, N Y T$

$N=I Y H(I N, I)$

80

85

90

05

$Y P=(F L O A T(N)-1 \cdot) \neq D Y+Y S T A R T$

$N X T=N \times T O T H(1)$

DO $101 \quad I M=1, N \times T$

$M=I X H(I M, I)$

$X P=(F L O A T(M)-1 \cdot) * D X+X S T A R T$

$J U=J U+5$

$R M N I=\operatorname{SQRT}((X-X P) \neq \# 2+(Y-Y P) * \# 2+Z \# \# 2)$

GMNI = CMPLX(COS(KAY\#RMNI), -SIN(KAY\#RMNI))/RMN I

$S I=C M P L X(K A Y * K A Y * R M N I * R M N 1-3,,-3, * K A Y * R M N 1) / R M N 1 * \# 4$

$A M N 1=(X-X P) \neq S 1$

$B M N 1=(Y-Y P) \neq S 1$

$C M N I=Z * S 1$

IF(NPRNT2,GT.0)HRITE $(6,31)$ RMN1,GMN1, S1,

IAMNI, BMN1, CMN1

31 FORMATI $\# 0$ RMNI $=$ *F10.6*, GMNI $=2 F 10.6 *, 51=2 F 10.61$ $1 * \quad A M N 1=* 2 F 10.6 *, B M N 1=2 F 10.6 *, C M N I=* 2 F 10.6)$ 
C COMPUTE COEFFICIENTS OF JX AND JY CORRESPONDING TO E SUB $X$ C

CJX1 $=-O M S 4 P * G M N 1 * D X D Y-S O 4 P E *$ (CMPLX(1 \&,KAY*RMN1)/RMNI**2

$1+(X-X P) * A M N 1) * G M N I * D X O Y$

CJYI $=-S O 4 P E *(Y-Y P) * A M N I * G M N 1 * D X D Y$

$A(I F, J U)=C J \times I$

$A(I F, J U+1)=C J Y I$

IF(NPRNT2, GT.0)WRITE $(6,2) \quad X P, Y P, C J X I, C J Y I$

2 FORMAT $* 0$ COEFFICIENTS OF JX AND JY CORRESPONDING TO

IE SUB $X$ AT $X P=* F 8.4 *, Y P=* F 8.4 * A N D \quad Z P=0 . \quad A R E * /(4 E 15.6))$

$C$

C

CJXI $1=-504 P E *(X-X P) * B M N 1 * G M N 1 * D X D Y$

CJYI $=-O M S 4 P * G M N 1 * D X D Y-S O 4 P E *(C M P L X(1$, KAY*RMNI) / RMNI**2

1

$A(I F+1, J U)=C J \times I$

$A(I F+1, J U+1)=C J Y I$

IF (NPRNT2.GT.0) HRITE $(6,3) \times P, Y P, C J X I, C J Y I$

3 FORMATI $* 0$ COEFFICIENTS OF JX AND JY CORRESPONDING TO

C

IE SUB Y AT $X P=* F 8.4 *, Y P=* F 8.4 * A N D \quad Z P=0$. ARE*/(4E15.6))

C

COMPUTE COEFFICIENTS OF JX AND JY CORRESPDNDING TO E SUB $Z$

CJXI $=-S O 4 P E *(X-X P) \neq C M N 1 * G M N 1 * D X O Y$

$C J Y 1=-S O 4 P E *(Y-Y P) * C M N 1 * G M N 1 * D X D Y$

$A(I F+2, J U)=C J X I$

$A(I F+2, J U+1)=C J Y I$

IF(NPRNT2.GT.0)WRITE $(6,4) \quad X P, Y P, C J X 1, C J Y I$

4 FORMATI $\$ 0$ COEFFICIENTS OF JX AND JY CORRESPONDING TO

IE SUB $Z$ AT $X P=* F 8.4 * Y P=\$ F 8.4 * A N D Z P=0$. $A R E * /(4 E 15.6) / 1$

c 101 CONTINUE

C COMPUTE COEFFICIENTS IN THE SECOND OR $X=0$ PLANE

C (SUBSCRIPT 2, NEAR YZ-PLANE, FRONT HALL)

C

DO $102 L=L S, L F$

$Z P=(F L O A T(L)-1) * D Z+.Z S T A R T$

NYT $E$ NYTOTH (L)

DO $102 \quad I N=1, N Y T$

$N=I Y H(I N, L)$

$Y P=(F L O A T(N)-1 \cdot) * D Y+Y S T A R T$

$J U=J U+5$

$R N L 2=S Q R T(X * * 2+(Y-Y P) * \# 2+(Z-Z P) * * 2)$

GNL $2=C M P L X(C O S(K A Y * R N L 2),-S I N(K A Y * R N L 2)) / R N L 2$

$S 2=C M P L X(K A Y * K A Y * R N L 2 \# R N L 2-3,,-3, \# K A Y * R N L 2) / R N L 2 \# \# 4$

$A N L 2=X * S 2$

$B N L 2=(Y-Y P) * S 2$

$C N L Z=(Z-Z P) * 52$

IF (NPRNT2.6T.0) WRITE $(6,32)$ RNL 2, GNL2, S2,

I ANL 2, BNL 2, CNL 2 
32 FORMAT $(* 0$ RNL2 $=$ F $10.6 *$, GNL2 $=\$ 2 F 10.6 *, S 2=* 2 F 10.61$ $1 * \quad A N L 2=2 F 10.6 *, B N L 2=2 F 10.6 *, C N L 2=2 F 10.61$

C

C C

C

C

C

C C

175

180

185

C

C COMPUTE COEFFICIENTS FOR THE THIRD OR $Y=0$ PLANE

C (SUBSCRIPT 3 , NEAR XZ-PLANE, RIGHT HALL)

C

DO $103 \mathrm{~L}=\mathrm{LS}, \mathrm{LF}$

$Z P=(F L O A T(L)-1 \cdot) \neq D Z+Z S T A R T$

NXTENXTOTH(L)

DO $103 \quad I M=1, N \times T$

$M=I X H(I M, L)$

$X P=(F L O A T(M)-1 \cdot) \neq D X+X S T A R T$

$J U \cdot J U+2$

RML $3=S Q R T((X-X P) * 2+Y * \$ 2+(Z-Z P) * 2)$

195
GML $3=C M P L X(C O S(K A Y * R M L 3),-S I N(K A Y * R M L 3)) / R M L 3$

$S 3=C M P L X(K A Y * K A Y * R M L 3 * R M L 3-3,,-3$. *KAY*RML 3$) / R M L 3 * 4$

$A M L 3=(X-X P) * S 3$

$B M L 3=Y * S 3$

$C M L 3=(Z-Z P) * S 3$ 
IF (NPRNT2,GT.0) HRITE $(6,33)$ RML 3, GML 3, S3,

IAML $3, B M L 3, C M L 3$

33 FORMAT $* 0$ RML 3 $*$ * $10.6 *, G M L 3=2 F 10.6 *, S 3=2 F 10.61$

C

$1 * \quad A M L 3=2 F 10.6 *, B M L 3=2 F 10.6 *, C M L 3=* 2 F 10.6)$

C COMPUTE COEFFICIENTS OF JX AND JZ CORRESPONDING TO E SUB $X$ C

CJX3 $=-0 M S 4 P * G M L 3 * 0 X 0 Z-S 04 P E *$ (CMPLX(1,,KAY*RML 3$) / R M L 3 * 2$

$1+(X-X P) * A M L 3) * G M L 3 * D X D Z$

$C J Z 3=-S O 4 P E *(Z-Z P) * A M L 3 * G M L 3 * 0 \times D Z$

210

$A(I F, J U)=C J \times 3$

$A(I F, J U+1)=C J Z 3$

IF(NPRNT2.GT.0)WRITE $(6,8) \quad \times P, Z P, C J \times 3, C J Z 3$

8 FORMAT $\$ 0$ COEFFICIENTS OF JX AND JZ CORRESPONDING TO

IE SUB $X$ AT $X P=\$ F 8.4 *, Y P=0$. AND $Z P=\$ F 8.4 * \triangle R E *((4 E 15.6))$

C

C COMPUTE COEFFICIENTS OF JX AND JZ CORRESPONOING TO E SUB Y

C

$C J \times 3=-504 P E *(X-X P) * B M L 3 * G M L 3 * D X D Z$

$C J Z 3=-S O 4 P E *(Z-Z P) * B M L 3 * G M L 3 * 0 \times D Z$

220

$A(I F+1, J U)=C J \times 3$

$A(I F+1, J U+1)=C J 23$

IF (NPRNT2, GT.0) HRITE $(6,9) \times P, Z P, C J \times 3, C J Z 3$

9 FORMATP $\$ 0$ COEFFICIENTS OF JX AND $J Z$ CORRESPONDING TO

225

IE SUB $Y$ AT $X P=F 8.4 *, Y P=0$. $\triangle N D \quad Z P=F 8.4 * A R E *((4 E 15.6))$

C

C COMPUTE COEFFICIENTS OF JX AND JZ CORRESPONDING TO E SUB $Z$

C

CJX3 $=-504 P E *(X-X P) * C M L 3 * G M L 3 * D \times D Z$

C JZ3 $=-O M S 4 P * G M L 3 * D X D Z-S D 4 P E *$ (CMPLX(1, KAY*RML 3)/RML $3 * * 2$

230

1 $+(Z-Z P) * C M(3) * G M L 3 * D \times D Z$

$A(I F+2, J U)=C J \times 3$

$A(I F+2, J U+1)=C J Z 3$

IF (NPRNT2.GT.0) HRITE $(6,10) \quad X P, Z P, C J \times 3, C J Z 3$

10 FORMAT $\$ 0$ COEFFICIENTS OF $J X$ AND $J Z$ CORRESPONDING TO

IE SUB $Z$ AT $X P=* F 8.4 *, Y P=0$. AND $Z P=* F 8.4 * A R E * /(4 E 15.6) / 1)$

103 CONTINUE

100 CONTINUE

$N A=J U+1$

$M A=I F+2$

IF(NPRNT3,GT.O) WRITE $(6,13)$ MA,NA

13 FORMAT( $* 0$ MA $\#$ *IS* NA $\$$ \# I5/)

CALL CORDCT(MOIMA,MA,NA,A, JPVT, OIAG)

IF (NPRNT4.GT.0) WRITE $(6,12)$ (DIAG(I), I =1,NA)

12 FORMAT( $\$$ ODIAGONAL $*(/(8 E 15.6)$ )

$C 1=C M P L X(.376681,-.004938)$

$D T O R=P I / 180$.

$F T O M=.304801$

$K A Y=177.72 * D T O R$

$X P P=10, * F T O M$

$Y P P=-32,5 * F T O M$

WRITE $(6,17)$ 
C

C READ THE DBSERVED FIELD DATA

C

265

270

275

280

285

295

$k X=0$

$K Y=1$

$K Z=1$

$K Y F=16$

IF $=-2$

DO $200 \quad I Z=L S, L F$

$Z=(F L O A T(I Z)-1 \cdot) * D Z+Z S T A R T$

IF(IZ.EO.2) GO TO 201

$Z D=.75 * F T O M$

$T C=80$. *DTOR

GO TO 202

$20120=2.25 * F T O M$

$T C=99$. *DTOR

202 NYTOT=NYTOTH(IZ)

DO 200 IN=1, NYTOT

$I Y=I Y H(I N, I Z)$

$Y=(F L D A T(I Y)-1 \cdot) * D Y+Y S T A R T$

NXTOT $=$ NXTOTH(IZ)

DO $200 I M=1, N X T O T$

$I X=I X H(I M, I Z)$

$X=(F L O A T(I X)-1) * D X+.X S T A R T$

$I F=I F+3$

$217 K X=K X+1$

IF $(K X . L E .13)$ GO TO 218

$K X=1$

$K Y=K Y+1$

IF(KY.LE.KYF) GO TO 218

$K Y=1$

$K Z=K Z+1$

$K Y F=11$

IF (KZ.GT.2) GO TO 219

14 FORMAT (6E10.3)

C

C

LSUBZ $=.56$

$\triangle M P X=\triangle M P X / L S U B Z$

$\triangle M P Y=A M P Y / L S U B Z$

$A M P Z=A M P Z / L S U B Z$

THET $A=90$. *DTOR-ATAN (ZD/RSUBT)

17 FORMATI/

IFIELD POINTS $\$ 1 / 3(3 X * A M P * 4 X * P H A S E * 1 X), 11 X * E$ SUB $Z * 1)$

218 READ $(5,14)$ AMPX,PHSX, AMPY,PHSY, AMPZ, PHSZ

IF $(I X, N E \bullet K X) \cdot O R \cdot(I Y \cap N E \bullet K Y) \cdot O R \cdot(I Z \cdot N E \cdot K Z))$ GO TO 217

C REMOVE DIRECT ANTENNA CONTRIBUTION FROM THE OBSERVED FIELD DATA

RSUBT $=S Q R T((X P P-X) * 2+(Y P P-Y) * * 2)$

RSUBV $=(C O S(T H E T A)-C 1) /(C D S(T H E T A)+C 1)$

$R=S Q R T(R S U B T * 2+Z D * 2)$

$A R G=K A Y * R+T C$

$E S U B Z=(1 .+R S U B V) * 18.515939 / R * C M P L X(C D S(A R G),-S I N(A R j))$ HRITE $(6,16)$ AMPX, PHSX, AMPY, PHSY, AMPZ, PHSZ, ESUBZ 
16 FORMAT(F $8.6, F 8.2, F 8.6, F 8.2, F 8.6, F 8.2,2 E 15.6)$

$E(I F)=A M P X * C M P L X(C O S(D T O R * P H S X), S I N(D T O R * P H S X))$

$E(I F+1)=A M P Y * C M P L X(C O S(D T O R * P H S Y), S I N(D T O R * P H S Y))$

$E(I F+2)=A M P Z * C M P L X(C O S(D T O R * P H S Z), S I N(D T O R * P H S Z))-E S U B Z$

200 CONTINUE

219 CALL CORSLT(MDIMA,MA,NA,A,JPVT,DIAG, E, XS,RESID)

RESID=RESID/FLOAT (MA)

IF (NPRNT5.GT.0) WRITE $(6,21)$ RESID

21 FORMAT ( / * RESIDUAL $\approx * E 15.6)$

WRITE $(6,18)$

18 FORMAT $(/ *$ CURRENT IN THE $2 P=0$ PLANE*14X*XP*6X*YP*15X $1 * J$ SUB $X * 24 X * J$ SUB $Y * / 1$

$J U=-1$

NYTOT $=$ NYTOTH $(I)$

NXTOT $=N X T O T H(1)$

DO 301 IN $=1$, NYTOT

$N=I Y H(I N, I)$

$Y P=(F L O A T(N)-1) * D Y+.Y S T A R T$

DO 301 IM=1,NXTOT

$M=I X H(I M, I)$

$X P=(F(D A T(M)-1) * D X+,X S T A R T$

$J U=J U+2$

HRITE $(6,22) \quad X P, Y P, X S(J U), X S(J U+1)$

HRITE $(7,23) \times S(J U), X S(J U+1)$

22 FORMAT $(2 F 8.4,4 E 15.6)$

23 FORMAT( 4E 15.6)

301 CONTINUE

HRITE $(6,19)$

19 FORMATI/ $*$ CURRENT IN THE $X P=0$ PLANE*/4X*YP*6X*2P*15X $1 * J$ SUB $Y * 24 X * J$ SUB $Z * / 1$

DO $302 L=L S, L F$

$Z P=(F L O A T(L)-1) * D Z+.Z S T A R T$

NYTOT $=$ NYTOTH(L)

DO 302 IN $=1$, NYTOT

$N=I Y H(I N, L)$

$Y P=(F L O A T(N)-1 \cdot) \notin D Y+Y S T A R T$

$J U=J U+2$

HRITE $(6,22) \quad Y P, Z P, X S(J U), X S(J U+1)$

HRITE $(7,23) \times S(J U), \times S(J U+1)$

302 CONTINUE

WRITE $(6,20)$

20 FORMATI/ $/$ CURRENT IN THE YP=0 PLANE*14X*XP*6X*2P*15X

$1 * J$ SUB $x * 24 x * J$ SUB $2 * 11$

DO $303 L=L S, L F$

$Z P=(F L O A T(L)-1 \cdot) * D Z+Z S T A R T$

NXTOT $=N X T O T H(L)$

DO $303 I M=1, N X T O T$

$M=I \times H(I M, L)$

$X P=(F L O A T(M)-1 \cdot) * D X+X S T A R T$

$J U=J U+2$

WRITE $(6,22) \times P, Z P, X S(J U), X S(J U+1)$

WRITE $(7,23) \times S(J U), \times S(J U+1)$

303 CONTINUE

STOP

END 
SUBROUTINE CORDCT(MDIMA, M, N, A, JPVT, DIAG)

C

SUBROUTINE CORDCT COMPUTES THE QR FACTDRIZATIDN OF THE MATRIX A

C WHICH CAN BE INPUT TO SUBROUTINE CORSLT IN ORDER TO COMPUTE A

C LEAST SQUARES SOLUTION TO THE EQUATIONS $A X=B$.

INPUT PARAYETERS

C

C

C

C

c

C

c

C

c

C

C

C

C

c

c

C

C

C

C

c

C

c

c

c

C

$\Delta$

CONTAINS THE UPPER TRIANGULAR MATRIX R AND INFORMATION THAT CAN BE USED TO EFFICIENTLY DETERMINE THE MATRIX

Q. THIS ARRAY IS INPUT TO SUBRDUTINE CORSLT WHEN A LEAST SQUARES SOLUTION IS COMPUTED.

JPVT

A SINGLY SUBSCRIPTED INTEGER ARRAY OF LENGTH N THAT CONTAINS INTERMEDIATE RESULTS THAT ARE INPUT TO SUBROUTINE CORSLT IF A LEAST SQUARES SOLUTION IS COMPUTED.

DIAG

A SINGLY SUBSCRIPTED COMPLEX ARRAY OF LENGTH N THAT

CONTAINS INTERMEDIATE RESULTS THAT ARE INPUT TO SUBROUTINE CQRSLT IF A LEAST SQUARES SOLUTIDN IS TO BE COMPUTED.

INTEGER JPVT (N)

COMPLEX A (MDIMA,N), DIAG(N), AHOLD

$D O 8 I=1, N$

$D I \Delta G(I)=0$.

JPVT II) =I

8 CONTINUE

OO I $J=1, N$

C

HRITE $(6,753) \mathrm{J}$

753 FORMAT (* J $=*$ I5)

$M M J=M-J+1$

EMAXN $=0$.

DO $2 K=J, N$

ENORM $=\operatorname{VNORM(MMJ,A}(J, K))$

IF(ENORM.LE.EMAXN) GO TO 2

JPVTHEK

EMAXN=ENORM

2 CONTINUE

IF(EMAXN.EQ.0.) RETURN

$J P V T(J)=J P V T H$

DO $3 I=1, M$ 


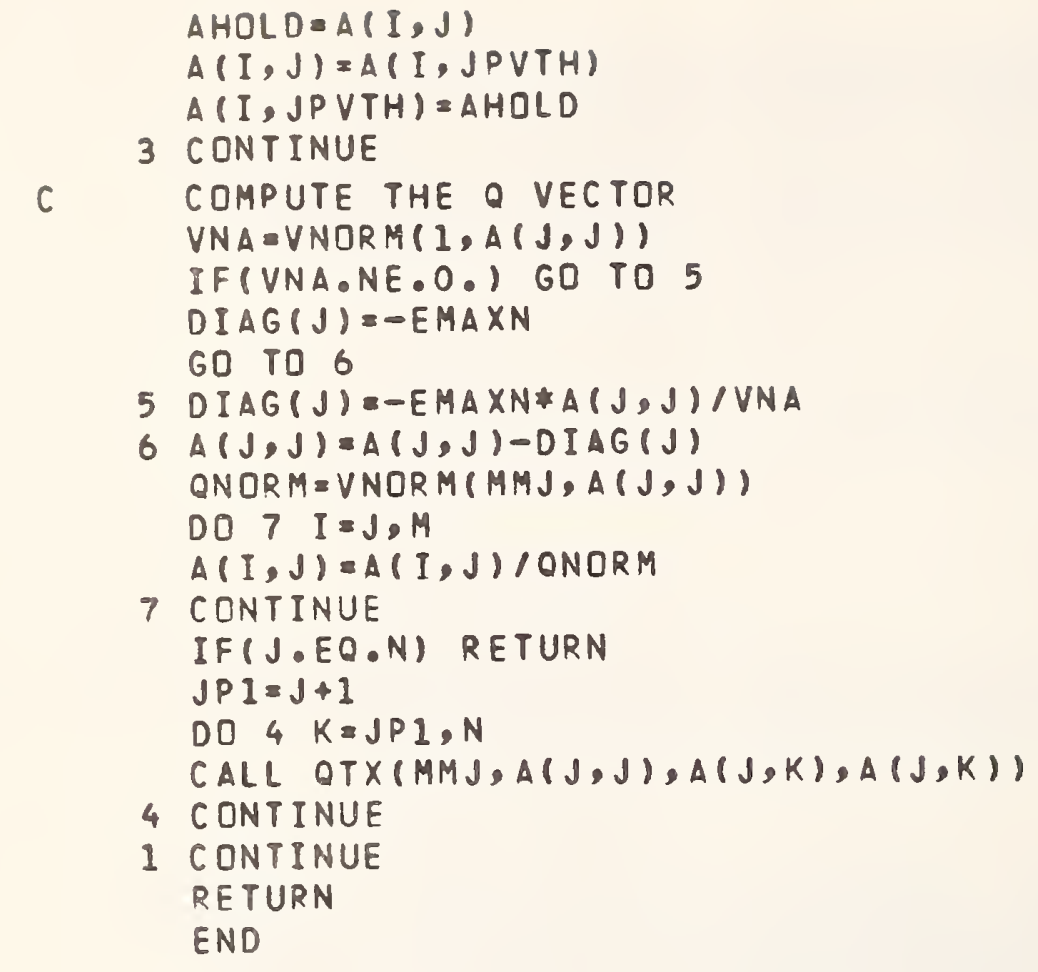

SUBROUTINE CQRSLT(MDIMA,M,N,A, JPVT, DIAG, B, X,RESID)

C

C SUBROUTINE CQRSLT COMPUTES THE LEAST SQUARES SOLUTION DF

C $A X=B$ USING THE QR DECOMPOSITION THAT IS COMPUTED BY

C SUBRDUTINE CORDCT.

C

C

C

C

INPUT PARAMETERS

MDIMA THE FIRST OR ROW DIMENSION OF A

M THE NUMBER OF COLUMNS DF A.

N THE NUMBER OF COLUMNS OF A.

A A THO DIMENSIONAL COMPLEX ARRAY THAT CONTAINS THE INFORMATION FOR THE QR DECOMPOSITION OF A THAT WAS COMPUTED BY SUBROUTINE CORDCT.

JPVT A SINGLY SUBSCRIPTED INTEGER ARRAY OF LENGTH N THAT CONTAINS RESULTS THAT WERE COMPUTED BY SUBROUTINE CORDCT.

DIAG A SINGLY SUBSCRIPTED COMPLEX ARRAY OF LENGTH N THAT CONTAINS RESULTS THAT WERE COMPUTED BY SUBROUTINE CORDCT.

B

A SINGLY SUBSCRIPTED COMPLEX ARRAY OF LENGTH M THAT CONTAINS THE RIGHT HANO SIDE OF THE EQUATIONS $\triangle X=B$. 


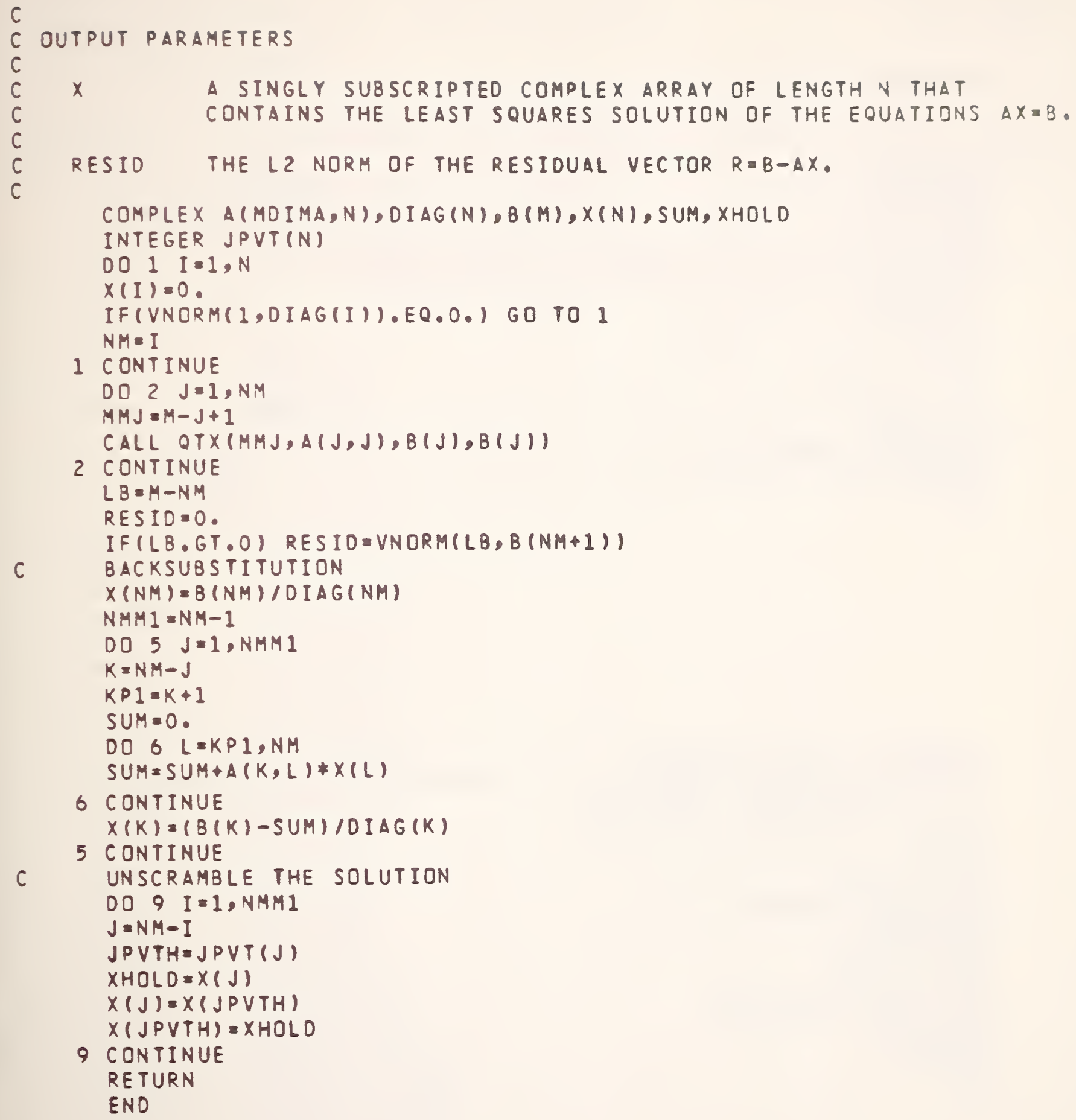

9 CONTINUE

RETURN

END 


\section{FUNCTION VNORM $(M, X)$}

C

C FUNCTION VNORM COMPUTES THE NORM OF THE M-VECTOR $X$.

C

COMPLEX X(M)

$X M A X=0$.

$001 I=1, M$

$X M A X=A M A X I(X M A X, A B S(R E A L(X(I))), A B S(A I M A G(X(I))))$

1 CONTINUE

VNORM $=0$.

IF (XMAX.EQ.0.) RETURN

SUM $=0$.

$002 I=1, M$

$S U M=S U M+(R E A L(X(I)) / X M A X) * * 2+(A I M A G(X(I)) / X M A X) * * 2$

2 CONTINUE

VNORM $=$ XMAX*SORT (SUM)

RETURN

END

SUBROUTINE QTX $(M, Q, X, Q X)$

C COMPUTE $Q$ TIMES $X$ WHERE $Q$ IS AN ELEMENTARY REFLECTOR AND

C $X$ IS AN ARBITRARY M-VECTOR.

COMPLEX Q(M),X(M),OX $(M), O H X$

$Q H X=0$.

$D O 1 I=1, M$

$Q H X=Q H X+C O N J G(O(I)) * X(I)$

1 CONTINUE

$\mathrm{OHX}=\mathrm{OHX}+\mathrm{QHX}$

$D O 2 I=1, M$

$Q X(I)=X(I)-Q H X * Q(I)$

2 CONTINUE

RETURN

END 


\section{A.2 FIELD Program}

1

5

10

15

20

25

30

35

40

PROGRAY FIELD (INPUT, OUTPUT, TADES = INPUT, TAPEG = OUTPUT)

C THIS PROGRAM READS COMPUTED CURRENTS AS DATA FROM FILE $123 C X X X$

C AND COMPUTES FIELD STRENGTHS AT A SELECTED FIELD POINT

COMPLEX OMS4P, SO4PE, GMN1, GNL2, GML3, S1, S2, S3, AMN1, ANL 2, \&4L 3 1 , BMN 1, BNL2, BML3,CMN1, CNL2,CML3,CJX1, CJX3,CJY1, CJY2 , E (3), CJZ?, CJZ3,C1, R SUBV, ESUBZ, XS $(24)$

INTEGER NXTOTH(2), NYTOTH(2), IXH(13,2), IYH(16,2)

REAL MU, KAY

NPRNTI $=0$

NPRNT2 $=0$

NPRNT3 $=0$

C NPRNTI CONTROLS PRINTING OF OMEGA,MU,EPS,KAY,OMS4P,SO4DE

C NPRNTZ CONTROLS PRINTINT DF XP, YP, ZP AND COEFFICIENTS

C NPRNT3 CONTROLS PRINTING OF MA,NA

$N \times T O T H(1)=2$

$N \times T \cap T H(2)=2$

NYTOTH $(1)=2$

NYTOTH $(2)=2$

$\operatorname{IXH}(1,1)=1$

I XH(1, 2) $=1$

$\operatorname{IXH}(2,1)=2$

$\operatorname{IXH}(2,2)=2$

$\operatorname{IYH}(1,1)=1$

$\operatorname{IYH}(1,2)=1$

$\operatorname{IYH}(2,1)=2$

$\operatorname{IYH}(2,2)=2$

$X=0.9144$

$Y=1.0668$

$Z=1.4478$

$D X=.6096$

$D Y=.6096$

$D Z=.4572$

$D X D Y=D X * D Y$

$D X D Z=D X * D Z$

$D Y D Z=D Y * D Z$

XSTART $=.9144$

YSTART $=1.0668$

ZSTART $=1.4478$

FREQ $=1.481 E 8$

$P I=4$ * $A T A N(1$.

$T P I=P I+P I$

OMEGA $=T P I * F R E O$

$M U=4, E-7 * P I$

$E P S=1 \cdot E-9 /(36, * P I)$ 
$L S=1$

$\ell F=2$

$K A Y=O M E G A * S Q R T$ (MU*EPS)

OMS 4P = CMPLX (0., OMEGA*MU/(4.\#PI))

SO4PE $=-C M P L X(0 ., 25 /(O M E G A * P I * E P S))$

50

IF (NPRNTI.GT.O)WRITE $(6,15)$ OMEGA,MU, EPS,KAY, OMS 4P, SO4PE

15 FORMAT( $* 0$ THE VARIABLES OMEGA, MU, EPS, KAY, OMS 4 P, SO4PE ARE ILISTED BELOW*/(8E15.6))

C

C

55

READ CURRENTS IN THE ZP=O PLANE, J SUB $X$ IND J SUB Y

$J U=-1$

NYTOTENYTOTH(1)

NXTOT $=$ NXTOTH(I)

DO 301 IN $=1$, NYTOT

DO $301 I M=1, N \times T O T$

$J U=J U+2$

READ $(5,22) \times S(J U), \times S(J U+1)$

22 FORMAT(4E15.6)

C

301 CONTINUE

C

READ CURRENTS IN THE XP=O PLANE, J SUB Y AND J SUB $Z$

C

DO $302 L=L S, L F$

NYTOT $=N Y T O T H(L)$

DO 302 IN $=1$, NYTOT

$J U=J U+2$

$R E A D(5,22) \times S(J U), \times S(J U+1)$

c

302 CONTINUE

C

C

READ CURRENTS IN THE YP=O PLANE, J SUB $X$ AND J SUB $Z$

DO $303 L=L S, L F$

NXTOT $=$ NXTOTH(L)

DO 303 IM $=1, N X T O T$

$J U=J U+2$

READ $(5,22) \times S(J U), \times S(J U+1)$

85

C

WRITE CURRENTS AND COORDINATES FOR CHECK

WRITE $(6,18)$

18 FORMAT $(1 *$ CURRENT IN THE $Z P=0$ PLANE* $14 X * X P * 6 x * Y P * 15 X$

$1 * J$ SUB $X * 24 X * J$ SUB $Y * / 1$

$J U=-1$

NYTOTENYTOTH(1)

NXTOT $=$ NXTOTH(1)

DO 321 IN=1, NYTOT

$N=I Y H(I N, I)$

$Y P=(F L D A T(N)-1) * D Y+.Y S T A R T$

DO 321 IM $=1, N X T O T$

$M=I X H(I M, 1)$

$X P=(F L O A T(M)-1) * D X+.X S T A R T$ 
$\mathrm{JU}=\mathrm{JU}+2$

WRITE $(5,322) \quad X P, Y P, X S(J U), \times S(J U+1)$

322 FORMAT $(2 F 8.4,4 E 15.6)$

321 CONTINUE

WRITE $(6,19)$

19 FORMAT $(1 *$ CURRENT IN THE $X P=0$ PLANE* /4X*YP*6X ZP*15X

$1 * J$ SUB $Y * 24 X * J$ SUB $Z * / 1$

DO $323 L=L S, L F$

$Z P=(F L O A T(L)-1 \cdot) * D Z+Z S T A R T$

NYTOT = NYTOTH(L)

DO 323 IN $=1$, NYTOT

$N=I Y H(I N, L)$

$Y P=(F L D A T(N)-1 \cdot) * D Y+Y S T A R T$

$J U=J U+5$

WRITE $(6,322) \quad Y P, Z P, X S(J U), X S(J U+1)$

323 CONTINUE

WRITE $(5,20)$

20 FORMATI / * CURRENT IN THE YP=0 PLANE*/4X*XP*6X*ZP*15X

$1 * J$ SUB $X * 24 x * J$ SUB $Z * 11$

$00324 L=L S, L F$

$Z P=(F L O A T(L)-1 \cdot) * D Z+Z S T A R T$

NXTOT $=N \times T D T H(L)$

DO 324 I $M=1, N \times T O T$

$M=I \times 4(I M, L)$

$X P=(F L O A T(M)-1.) \neq D X+X S T A R T$

$J U=J U+5$

WRITE $(6,322) \quad \times P, Z P, \times S(J U), \times S(J U+1)$

324 CONTINUE

I $F=1$

$E(I F)=0$.

$E(I F+1)=0$.

$E(I F+2)=0$.

IF(NPRNT?.GT.0) WRITE $(6,1) \quad X, Y, Z$

1 FORMAT $/ /$ ITHE FOLLOWING COEFFICIENTS CORRESPOND TO THE

IFIELD POINT \#/* $x=* F 8.4 *, Y=* F 8.4 *, Z=\# F 8.4 / 1$

135

C

C COMPUTE CDEFFICIENTS IN THE FLOOR OR $Z=0$ PLANE

C (SUBSCRIPT I, BOTTOM XY-PLANE, FLOOR)

C

$J U=-1$

NYT $=$ NYTOTH (1)

DO 101 IN $=1$,NYT

140

$N=I Y H(I N, I)$

$Y D=(F L D A T(N)-1 \cdot) * D Y+Y S T A R T$

$N \times T=N \times T O T H(1)$

DO 101 IM $=1, N \times T$

$M=I X H(I M, 1)$

145

$X P=(F L D A T(M)-1 \cdot) * D X+X S T A R T$

$J U=J U+2$

RMNI $=S \partial R T((X-X P) * 2+(Y-Y P) * * 2+Z * \neq 2)$

GMNI $=C M P L X(C O S(K A Y * R M N 1),-S I N(K A Y * R M N I)) / R M N I$

$S I=C M P L X(K A Y * K A Y * R M N I * R M N 1-3 .,-3 . * K A Y * R M N 1) / R M N I * *$ 
$A M N I=(X-X P) * S 1$

$B M N I=(Y-Y P) * S I$

$C M N I=Z * S 1$

C

C COMPUTE COEFFICIENTS OF JX AND JY CORRESPONDING TO E SUB $X$ $\mathrm{C}$

CJXI $=-D M S 4 P * G M N I * D \times D Y=S O 4 P E *(C M P L \times(1, . K A Y * R M N I) / R M N I * * 2$

$1+(X-X P) * A M N I) * G M N I * D X D Y$

$C J Y I=-S 04 P E *(Y-Y P) * A M N I * G M N I * D X D Y$

$E(I F)=E(I F)+C J \times I * X(J U)+C J Y I * X S(J U+I)$

160

IF (NPRNT? GT.0)WRITE $(6,2) \quad X P, Y P, C J \times I, C J Y 1$

2. FORMAT( $\#$ COEFFICIENTS OFF JX AND JY CORRESPONDINU TO

IE SUB $X \triangle T X P=* F 8.4 *, Y P=\$ F 8.4$ AND $Z P=0 . \quad \triangle R E * /(4 E 15.6))$

C

C

165

C

COMPUTE COEFFICIENTS OF JX AND JY CORRESPDNDING TD E SUB Y

C JXI $=-S 04 P E *(X-X P) * B M N I * G M N I * D X D Y$

CJYI $=-D M S 4 P * G M N I * D X D Y-S D 4 P E *(C M P L X(1$, , KAY*RMNI)/RMNI**2

1 $+(Y-Y ?) * B M V I) * G M N I * D X D Y$

$E(I F+1)=E(I F+1)+C J \times I * X S(J U)+C J Y I * X(J U+1)$

IF (NPRNT2.GT.0)WRITE $(6,3) \times D, Y P, C J \times I, C J Y I$

3 FORMAT $\$ 0$ COEFFICIENTS OF $J X$ AND JY CORRESPONDING TO

IE SUB Y AT $X P=* F 8.4 *, Y P=* F 8.4 *$ AND $Z P=0 . \quad A R E * /(4 E 15.6)$ )

C

C COMPUTE COEFFICIENTS OF JX AND JY CORRESPONDING TO E SUB $Z$

$C J \times I=-S D 4 P E *(X-X P) * C M N I * G M N I * D X D Y$

CJYI $=-S 04 D E *(Y-Y P) * C M N I * G M N I * D X D Y$

$E(I F+2)=E(I F+2)+C J X I * X S(J U)+C J Y I * X S(J U+1)$

IF(NPRNT2.GT.0)WRITE $(6,4) \times P, Y P, C J \times 1, C J Y I$

180

185

$\mathrm{C}$

4 FORMAT $* 0$ COEFFICIENTS OF JX AND JY CORRESPONDING TO

IE SUB $Z$ AT $X P=* F 8.4 *, Y P=* F 8.4 * \quad A N D \quad Z P=0 . A R E * /(4 E 15.6) / 1$ 101 CONTINUE

C COMPUTE COEFFICIENTS IN THE SECOND OR $x=0$ PLANE

C (SUBSCRIPT 2, NEAR YZ-PLANE, FRONT WALL)

C

$D 0102 L=L S, L F$

ZP $=(F L O A T(L)-1) * D Z+.Z S T A R T$

NYT $=$ NYTOTH(L)

190

DO 102 IN $=1, N Y T$

$N=I Y H(I N, L)$

$Y P=(F L O A T(N)-1 \cdot) * D Y+Y S T A R T$

$J U=J U+5$

$R N L 2=S Q R T(X * * 2+(Y-Y P) * * 2+(Z-Z P) * * 2)$

195

$G N L Z=C M P L X(C O S(K A Y * R N L 2),-S I N(K \Delta Y * R N L 2)) / R N L 2$

$S 2=C M P L X(K A Y * K A Y * R N L 2 * R N L 2-3,,-3 . * K \Delta Y * R N L 2) / R N L 2 * * 4$

$A N L 2=X * S 2$

$B N L 2=(Y-Y P) * S 2$

$C N L 2=(Z-Z P) * S 2$

200

C

C COMPUTE CDEFFICIENTS OF JY AND JZ CORRESPONDING TO E SUB $X$

C 
CJYZ $=-S 04 P E *(Y-Y P) * A N L 2 * G N L Z * D Y D Z$

$C J Z 2=-504 P E *(Z-Z P) * A N L 2 * G N L 2 * D Y D Z$

$E(I F)=E(I F)+C J Y Z * X S(J U)+C J Z 2 * \times S(J U+1)$

IF(NPRNT2.GT.0)WRITE $(6,5)$ YP, ZP, CJYZ,CJZZ

5 FORMAT $(* 0$ COEFFICIENTS OF JY AND JZ CORRESPONDING TO

IE SUB $X$ AT $X P=0 ., \quad Y P=* F 8.4 * \quad \triangle N D \quad Z P=* F 8.4 * \quad \triangle R E * /(4 E 15.5))$

CJY2 $=-3 M S 4 P * G N L 2 * D Y D Z-S O 4 P E *(C M P L X(1, K A Y * R N L 2) / R N L ?$ \# 2

$1+(Y-Y P) * B N L 2) * G N L 2 * D Y D Z$

$C J Z 2=-S D 4 P E *(Z-Z P) * B N L 2 * G N L 2 * D Y D Z$

215

$E(I F+1)=E(I F+1)+C J Y Z * X S(J U)+C J Z 2 * \times S(J U+1)$

IF (NPRNT2,GT.0)WRITE $(6,6)$ YP, ZP, CJYZ,CJZZ

6 FORMAT $(* 0$ COEFFICIENTS DF JY AND JZ CORRESPONDING TJ

C

IE SUB Y $\triangle T \quad X P=0 ., Y P=\# F 8.4 * \triangle N D Z P=\# F 8.4 * \triangle D E \# /(4 E 15.6) 1$

$\mathrm{C}$

C COMPUTE COEFFICIENTS FOR THE THIRD OR Y $=0$ PLANE

230

$C J Y Z=-S D 4 P E *(Y-Y P) * C N L Z * G N L Z * D Y D Z$

CJZ2 $=-D M S 4 P * G N L Z * D Y D Z-S O 4 P E *(C M P L X(1,, K A Y * R N L 2) / R N L 2 \neq 2$

1 $+(Z-Z P) * C N L 2) * G N L 2 * D Y D Z$

$E(I F+2)=E(I F+2)+C J Y Z * \times S(J U)+C J Z 2 * \times S(J U+I)$

IF(NPRNT2.GT.0)WRITE $(6,7)$ YP, ZP, CJYZ, CJZZ

7 FORMAT( 0 COEFFICIENTS DF JY AND JZ CORRESDONDING TO

IE SUB Z AT $X P=0 ., Y P=* F 8.4 *$ AND $Z P=\$ F 8.4 * A R E * /(4 E 15.5) / 1$ 102 CONTINUE

C (SUBSCRIPT 3, NEAR XZ-PLANE, RIGHT WALL)

C

DO $103 L=L S, L F$

$Z P=(F L O A T(L)-1 \cdot) \neq D Z+Z S T A R T$

$N X T=N X T O T H(L)$

DO 103 IM $=1, N \times T$

$M=I X H(I M, L)$

$X P=(F L D A T(M)-1) * D X+.X S T A R T$

$J U=J U+2$

$R M L 3=S O R T((X-X P) \neq * 2+Y * * 2+(Z-Z P) * * 2)$

GML $3=C M P L X(C O S(K A Y * R M L 3),-S I N(K A Y * R M L 3)) / R M L 3$

$S 3=C M P L X(K A Y * K A Y * R M L 3 * R M L 3-3 .,-3$. \#KAY*RML3)/RML3*\#4

$\triangle M L 3=(X-X P) \neq S 3$

245

$B M L 3=Y * S 3$

$C M L 3=(Z-Z P) * S 3$

C

C COMPUTE COEFFICIENTS OF JX AND JZ CORRESPONDING TC E SUZ $X$

CJX3 $=-D M S 4 P * G M L 3 * D X D Z-504 P E *(C M P L X(1, K A Y * F M L 3) / R, Y L 3 * 2$

1 $+(X-X P) * A M L 3) * G M L 3 * D \times D Z$

CJZ3 $=-S 04 P E *(Z-Z P) * A M L 3 * G M L 3 * D \times D Z$

$E(I F)=E(I F)+C J \times 3 * \times S(J U)+C J Z 3 * \times S(J U+1)$

IF (NPRNT2.GT.0)WRITE $(6,8) \times P, Z P, C J \times 3, C J Z 3$ 
8 FORMAT 1 *O COEFFICIENTS OF JX AND JZ CORRESPONDING TO IE SUB $X \triangle T X P=* F 8.4 *, Y P=0$. $\triangle N D Z P=* F 8.4 * A R E *((4 E 15.6))$

C C

COMPUTE COEFFICIENTS OF JX ANO JZ CORRESPONDING TO E SUB Y

$C J \times 3=-S 04 P E *(X-X P) * B M L 3 * G M L 3 * 0 \times 0 Z$

$C J Z 3=-S 04 P E *(Z-Z P) * B M L 3 * G M L 3 * D \times D Z$

$E(I F+1)=E(I F+1)+C J \times 3 * \times S(J U)+C J Z 3 * \times S(J U+1)$

IF (NPRNT2.GT.0)WRITE $(6,9) \quad X P, Z P, C J \times 3, C J Z 3$

9 FORMAT $(* 0$ COEFFICIENTS OF JX AND JZ CORRESPONDING TD

265

IE SUB Y $\triangle T$ T $X P=* F 8.4 *, Y P=0$. $\triangle N D Z P=* F 8.4 * \quad \triangle R E * /(4 E 15.6))$

C

C C

CJX3 $=-S 04 P E *(X-X P) * C M L 3 * G M L 3 * D X D Z$

$C J Z 3=-D M S 4 P * G M L 3 * D X D Z-S D 4 P E *(C M P L X(1, K A Y * R M L 3) / R M L 3 * 2$

1 $+(Z-Z P) * C M(3) * G M L 3 * D \times D Z$

$E(I F+2)=E(I F+2)+C J \times 3 * \times S(J U)+C J Z 3 * \times S(J U+1)$

IF(NPRNT2.GT.0) WRITE $(6,10) \quad X P, Z P, C J \times 3, C J Z 3$

10 FORMATI $\$ 0$ CDEFFICIENTS OF JX AND JZ CORRESPONDING TO

IE SUB $Z$ AT $X P=* F 8.4 *, Y P=0$. $\triangle N D Z P=* F 8.4 * A R E * /(4 E 15.6) / 1)$

103 CONTINUE

100 CONTINUE

$N A=J U+1$

$M A=I F+2$

280

IF(NPRNT3.GT.0) WRITE $(6,13)$ MA,NA

C

13 FORMAT(*0 MA $=* I 5 * \quad N A=* I 5 /)$

ADO DIRECT ANTENNA CONTRIBUTION TO E SUB $Z$

$C 1=C M P L \times(.376681,-.004938)$

$D T O R=P I / 180$.

FTOM $\approx .304801$

$K A Y=177.72 \nleftarrow D T D R$

$X P P=10 . F T O M$

$Y P P=-32 \cdot 5 * F T O M$

$Z D=7-4$ * FTOM

$T C=(41.5573 * Z+19.8333) * D T O R$

$R S U B T=S Q R T((X P P-X) * * 2+(Y P P-Y) * 2)$

THETA $=90$. *DTOR-ATAN $(Z D / R S U B T)$

295

RSUBV $=(C D S(T H E T A)-C 1) /(C O S(T H E T A)+C 1)$

$R=S O R T(R . S U B T * 2+Z D * 2)$

$A R G=K A Y \# R+T C$

ESUBZ $=(1 .+R S U B V) * 18.515939 / R * C M P L \times(C O S(A R G),-S I N(A R G))$

$E(I F+2)=E(I F+2)+E S \cup B Z$

C C WRITE E SUB $x$, E SUB $Y$, AND E SUB $Z$ AT THE FIELD POINT C

WR I TE $(5,17)$

17 FORMATI $/ *$ E SUB $X$, E SUB $Y$, AND E SUB $Z$ AT THE FIELD

IPOINT*//*X*7X*Y*7X*Z*3X,3(5X*AMP*4X*PHASE*1X)/1)

$\triangle M P X=C A B S(E(I F))$

$A M P Y=C A B S(E(I F+1))$ 
$\triangle M P Z=C A B S(E(I F+2))$

DHSX = $\triangle T A N 2$ ( $\triangle$ IMAG(E(IF)), REAL (E (IF)) ) / DTOR

PHSY $=A T A N 2(A I M A G(E(I F+1)), R E A L(E(I F+1))) / D T O R$

PHSZ $=\Delta T A N ?(A I M A G(E(I F+2)), R E A L(E(I F+2))) / D T D R$

WRITE $(6,16) X, Y, Z, \triangle M P X, P H S X, \triangle M P Y, D H S Y, \triangle M D Z$, DHSZ

16 FOPMAT(3F8.4,F10.6,F8.2,F10.6,F8.2, F10.6,F8.2)

STOP

$315 \quad$ END 


\section{B. 1 CURRENT and FIELD Programs}

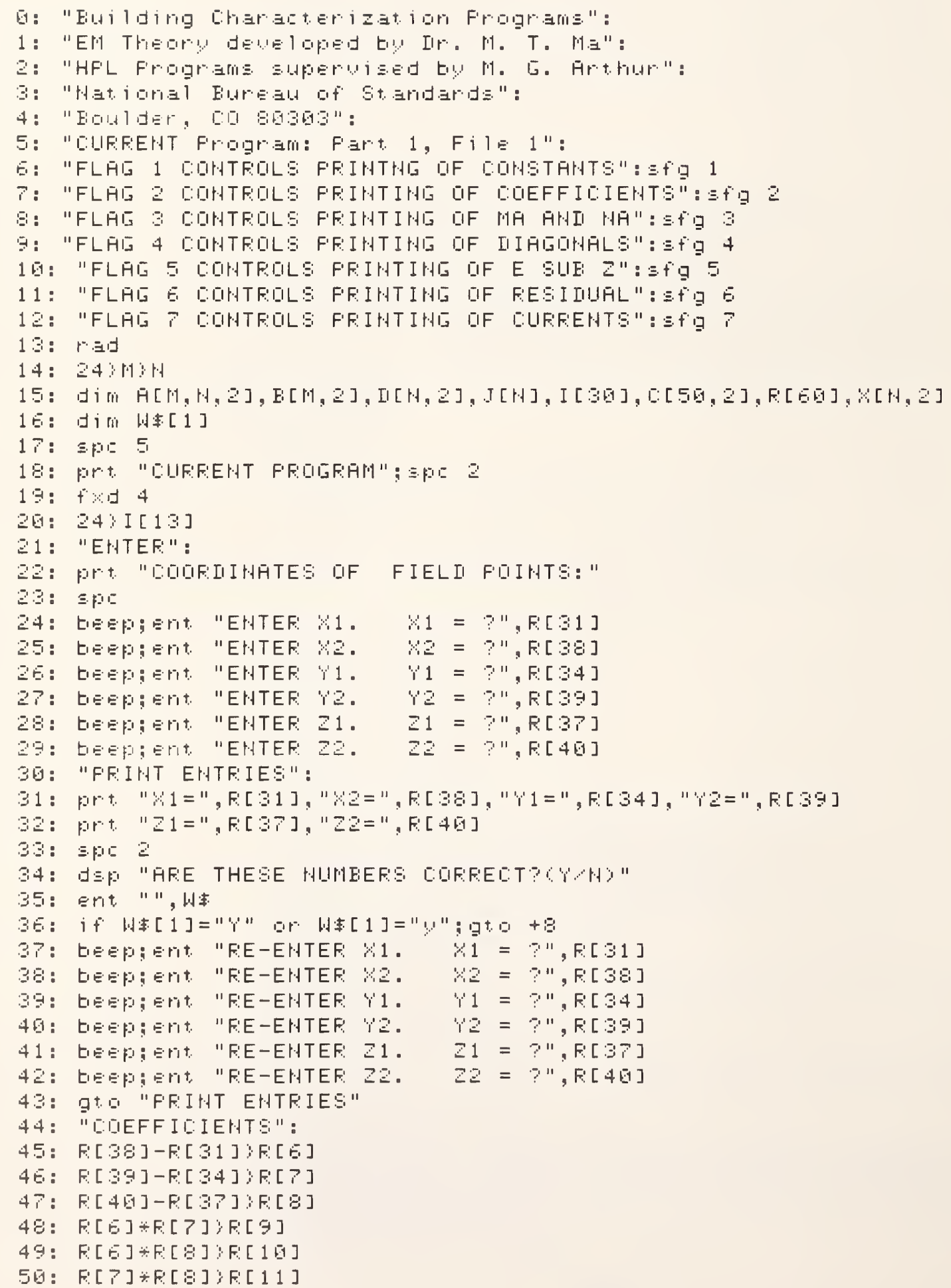




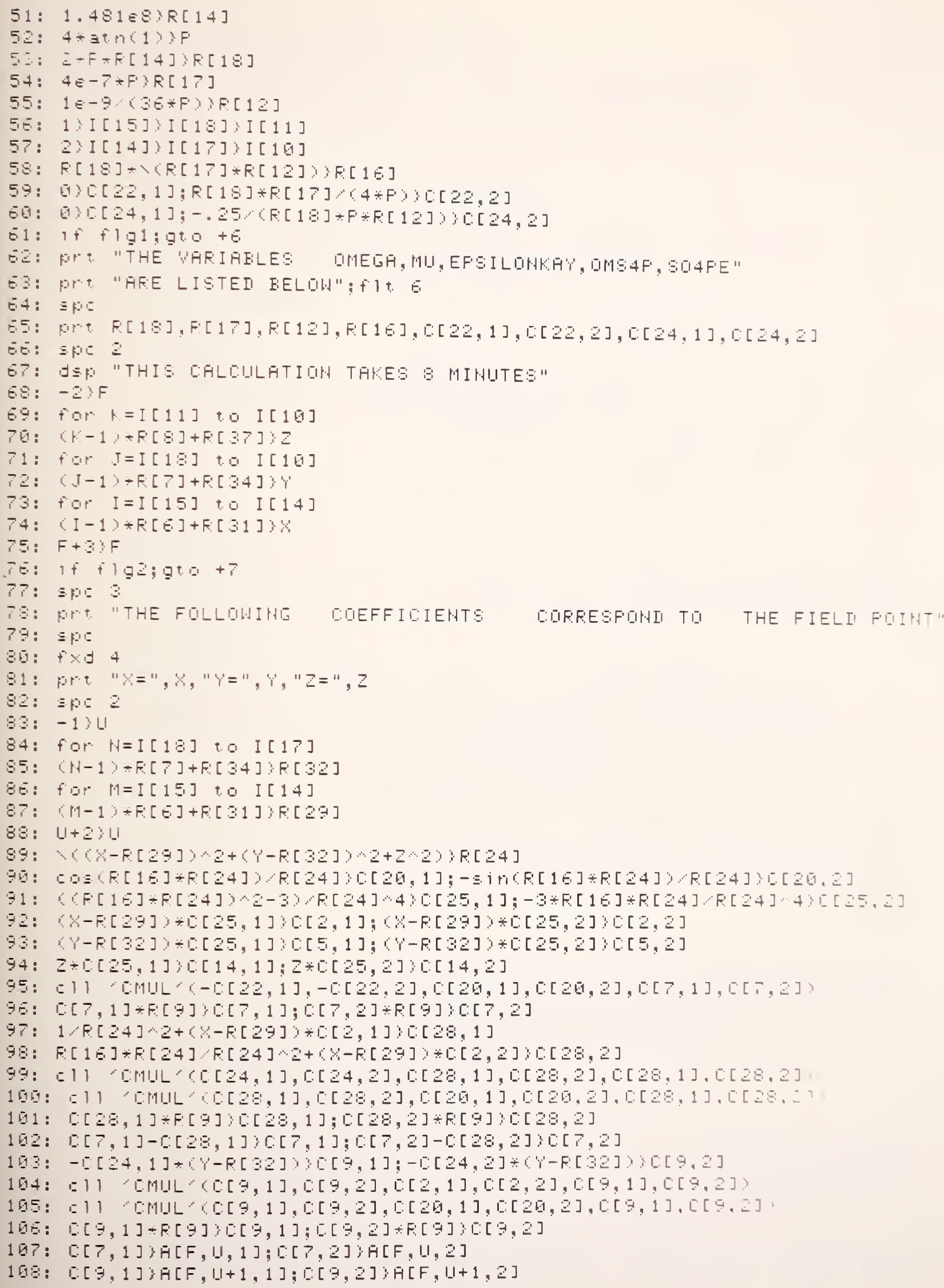


169: if flge; gto + 8

$110: 350$

111: Fro "COEFFIEIEHTS DIF JU AHI TY"

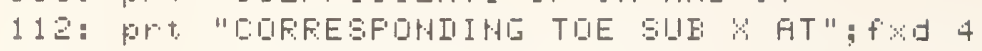

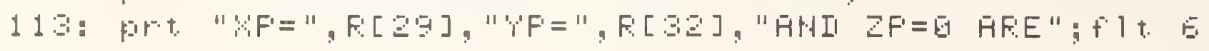

$114: \quad 300$

$115:$ prot. $[:[7,1],[:[7,2],[:[9,1],[[9,2]$

$11 \mathrm{~B}:$ : Fic

$117:-[[24,1] \div(\%-F[29])[[7,1] ;-[[24,2] \div(x-6:[29])[1], 2]$

$115: 511$ [

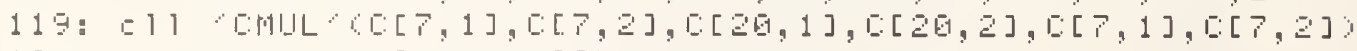

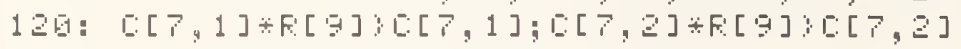

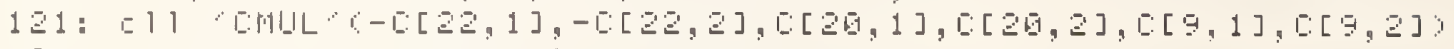

$122:[[9,1] \div[\because],[9,1] ;[[9,2] \div F[9] ;[[9,2]$

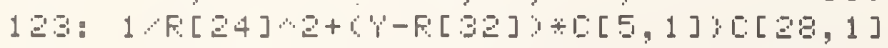

$124: F[1 E] \div F[24] F[24] 2+Y-F[32] \div[: 5,2]:[2,2,2]$

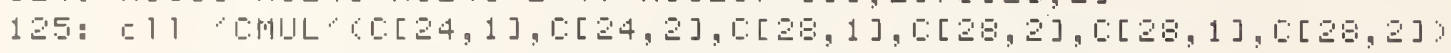

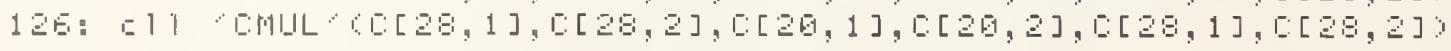

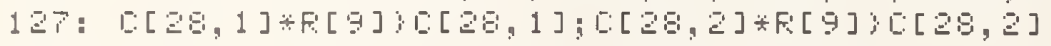

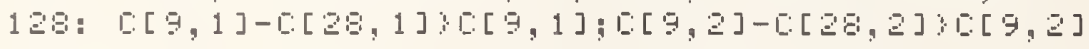

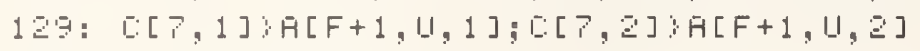

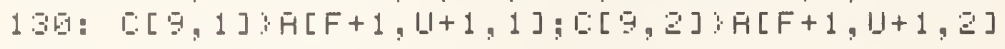

$131:$ if $f$ lg2; gto $+B$

132 : $\equiv$ :

133 pr. "DIEFFILIEHTS DIF T\% HWI JU"

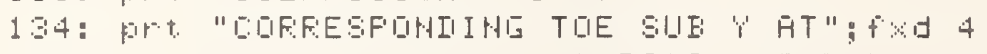

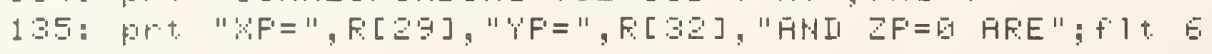

$136: \equiv$ :

$137: p, r .[-[7,1],[:[7,2],[[9,1],[:[9,2]$

$138: \quad \equiv$

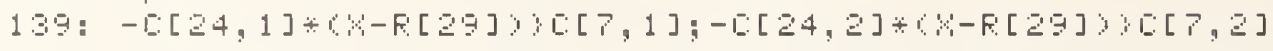

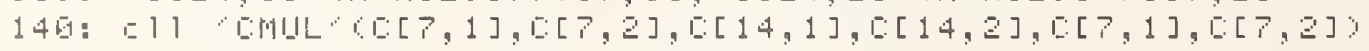

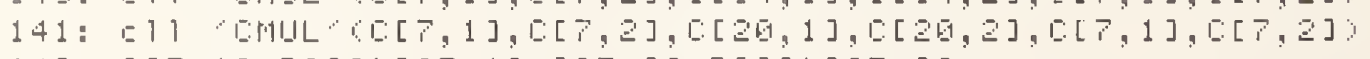

$142:[[;, 1] \div[:[9])[[7,1] ;[:[7,2] \div[[]][[7,2]$

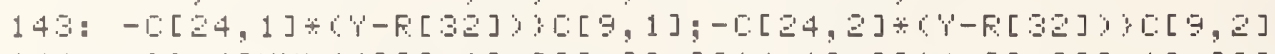

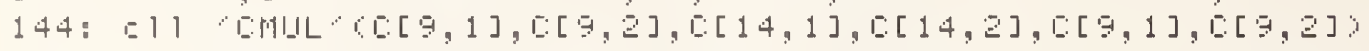

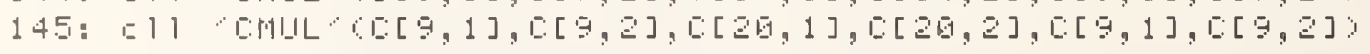

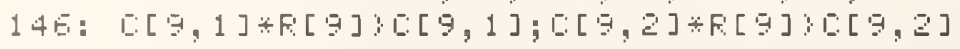

$147:[[7,1]) A[F+2,1,1] ;[[7,2]) H[F+2,1,2]$

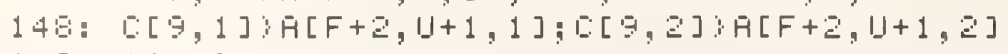

$149:$ if $f 192 ; g t a+g$

15日: $\equiv 50$

151: Frt "LDEFFIEIEHTS DF JY RHI I'"

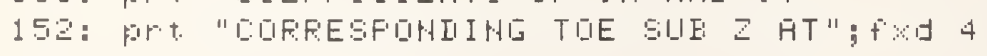

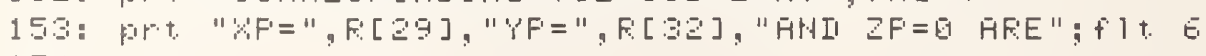

$154: \quad 5$ :

15:5: prot $[[7,1],[[7,2],[:[9,1],[:[9,2]$

$150: 5$ : 5

157 : rext in

1.5e: riset H

159: for $L=I[11]$ t. 0 I [ 1 1 1 $]$

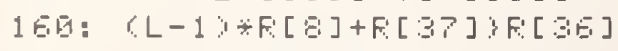

1E1: for $H=I\left[1 \varepsilon^{2}\right]$ to I [ 17$]$

$1 E:(4-1) \div[Y]+R[34) R[3]$

1E: $\quad(1+2 ; 1)$

1E4:

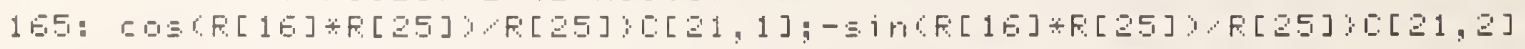

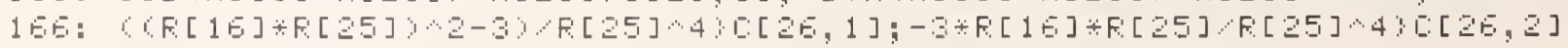


1ET: $x+[[26,1]\}[[3,1] ; X *[[26,2] ;[[3,2]$

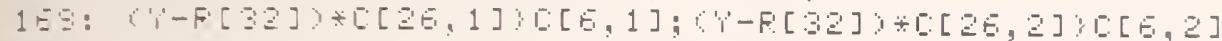

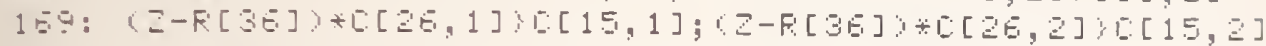

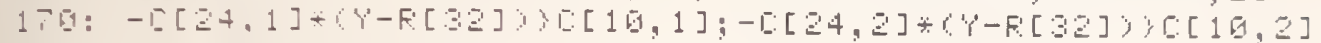

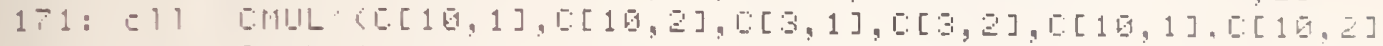

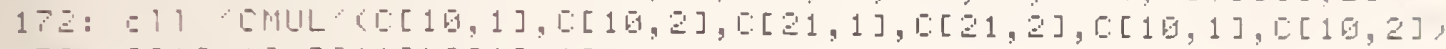

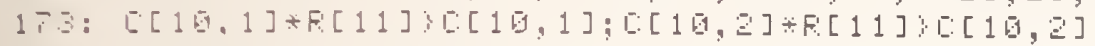

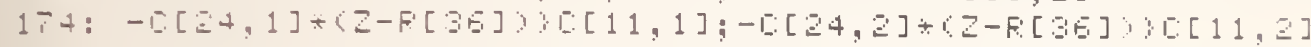

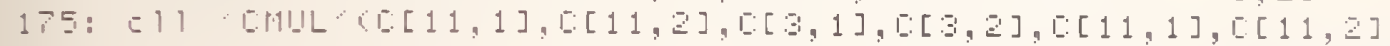

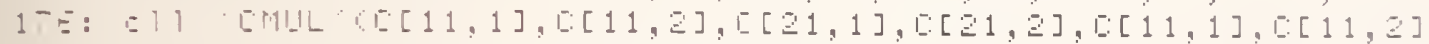

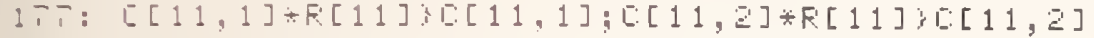

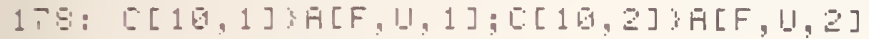

$17:[[11,1]: H[F,(I+1,1] ;[[11,2]) F[F, 11+1,2]$

1ह日: $1+f$ f

181: $\equiv$

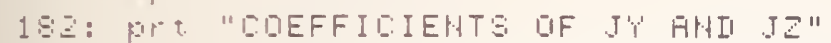

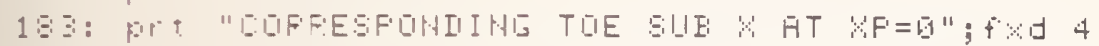

184: Pr. "YF=",F[SZ], "HHII ZF=",F[SE], "HFE";flt E

15: 500

$1 E:$ :

$187: \equiv$

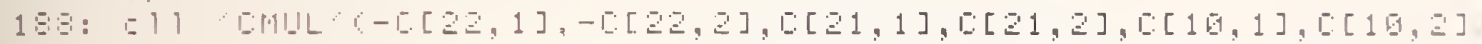

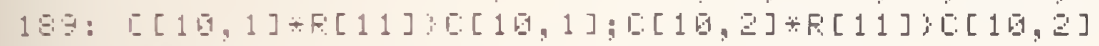

$19 B: 1$ F [ 25$] 2+(Y-F[3]) \div[E, 1])[2 E, 1]$

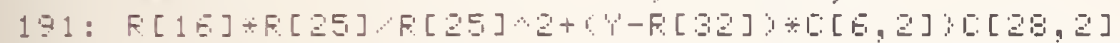

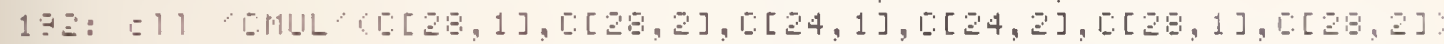

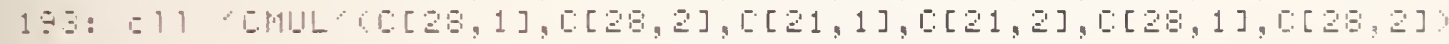

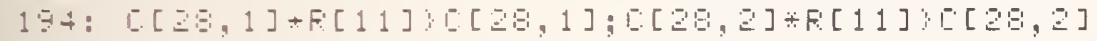

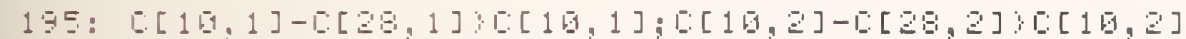

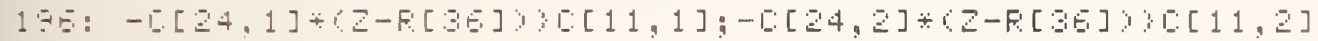

$15:[1,[M I L C[11,1],[[11,2],[[E, 1],[[E, 2],[[11,1],[[11,2]$

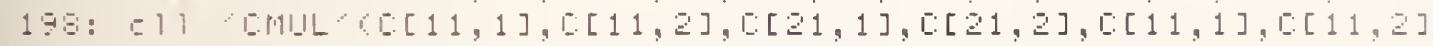

$19:[[11,1] * F[11] ;[[11,1] ;[[11,2] * F[11]\}[[11,2]$

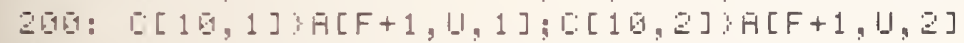

$201:[[11,1]: A[F+1, L I+1,1] ; C[11,2]: A[F+1, L I+1,2]$

26z: if flgz; gto $+\mathrm{B}$

201: $\equiv$

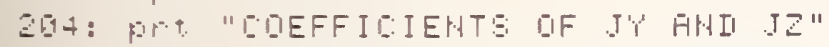

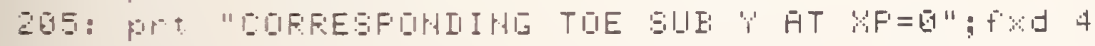

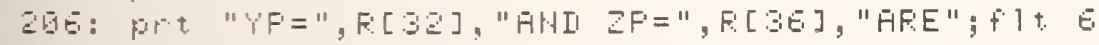

267: 5 :

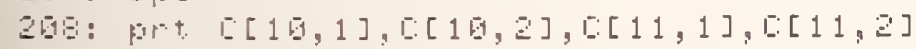

$209: 35$

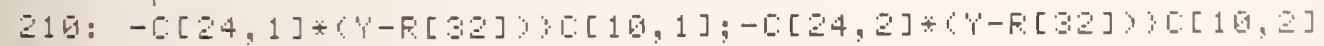

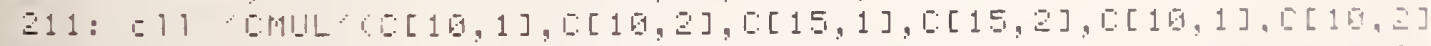

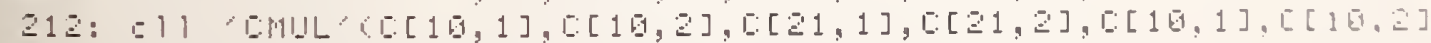

$213:[[15,1] * F[11] ;[[1], 1] ;[[15,2] * F[11] ;[:[15,2]$

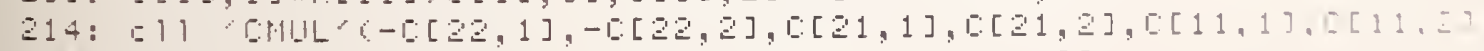

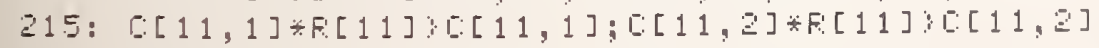

21E: $1: F[25] 2+(Z-F[3]] \div[15,1]:[28,1]$

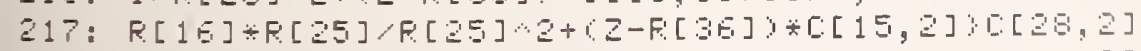

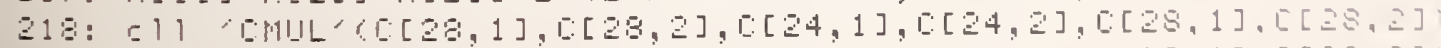

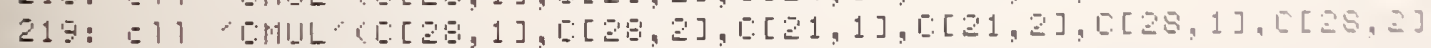

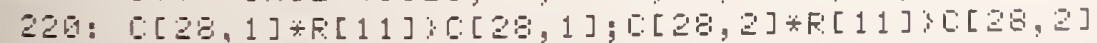

$221:[[11,1]-[:[2,1]:[11,1] ;[[11,2]-[[2], 2],[[11,2]$

$222:[[1], 1] ; A[F+2,1,1] ;[[16,2]) H[F+2, U, 2]$

$223:[[11,1] ; H[F+2,1,1+1,1] ; 1:[11,2]: H[F+2, U 1+1,2]$

$224:$ if $f 192 ; g+6+8$ 


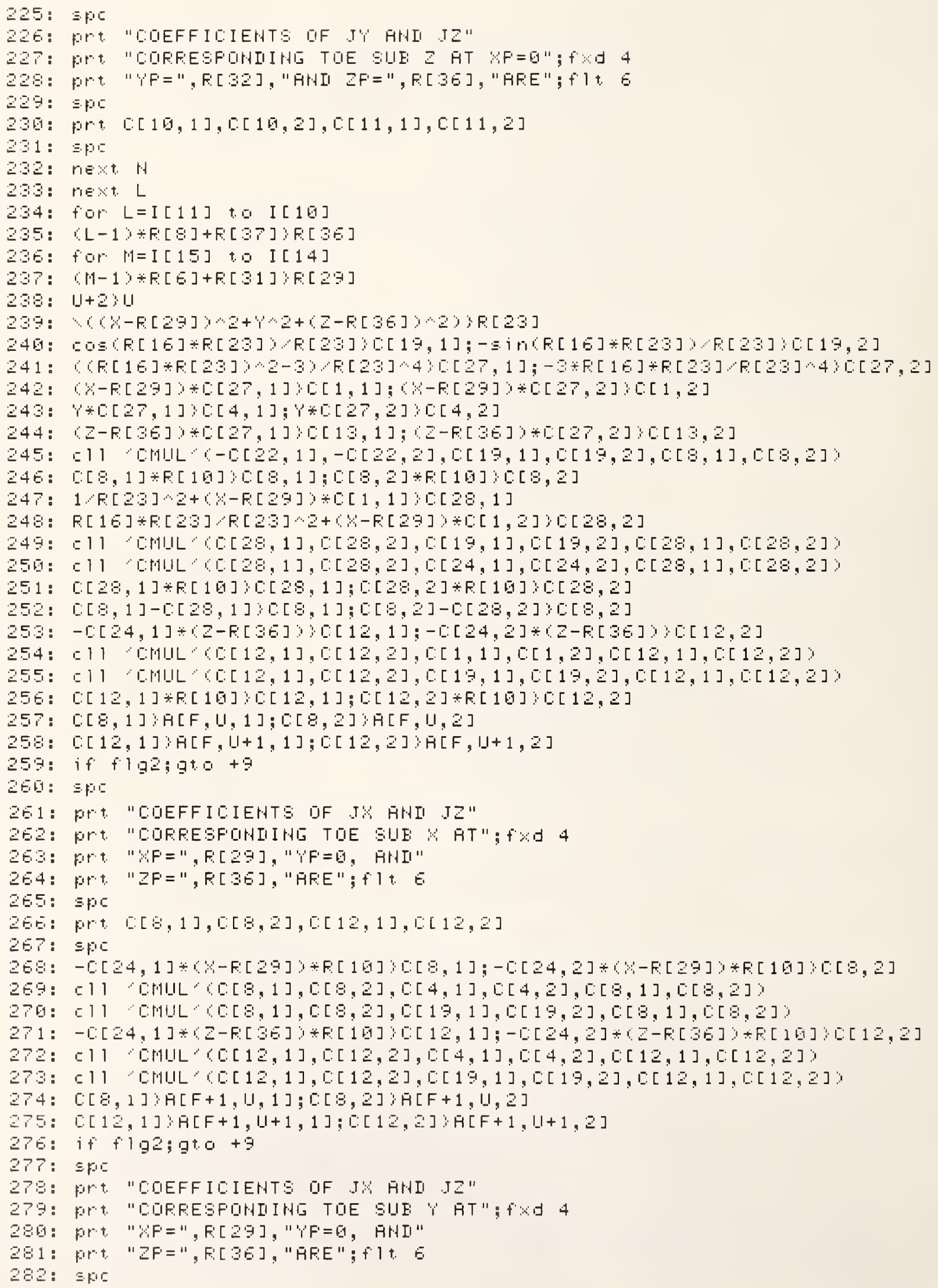




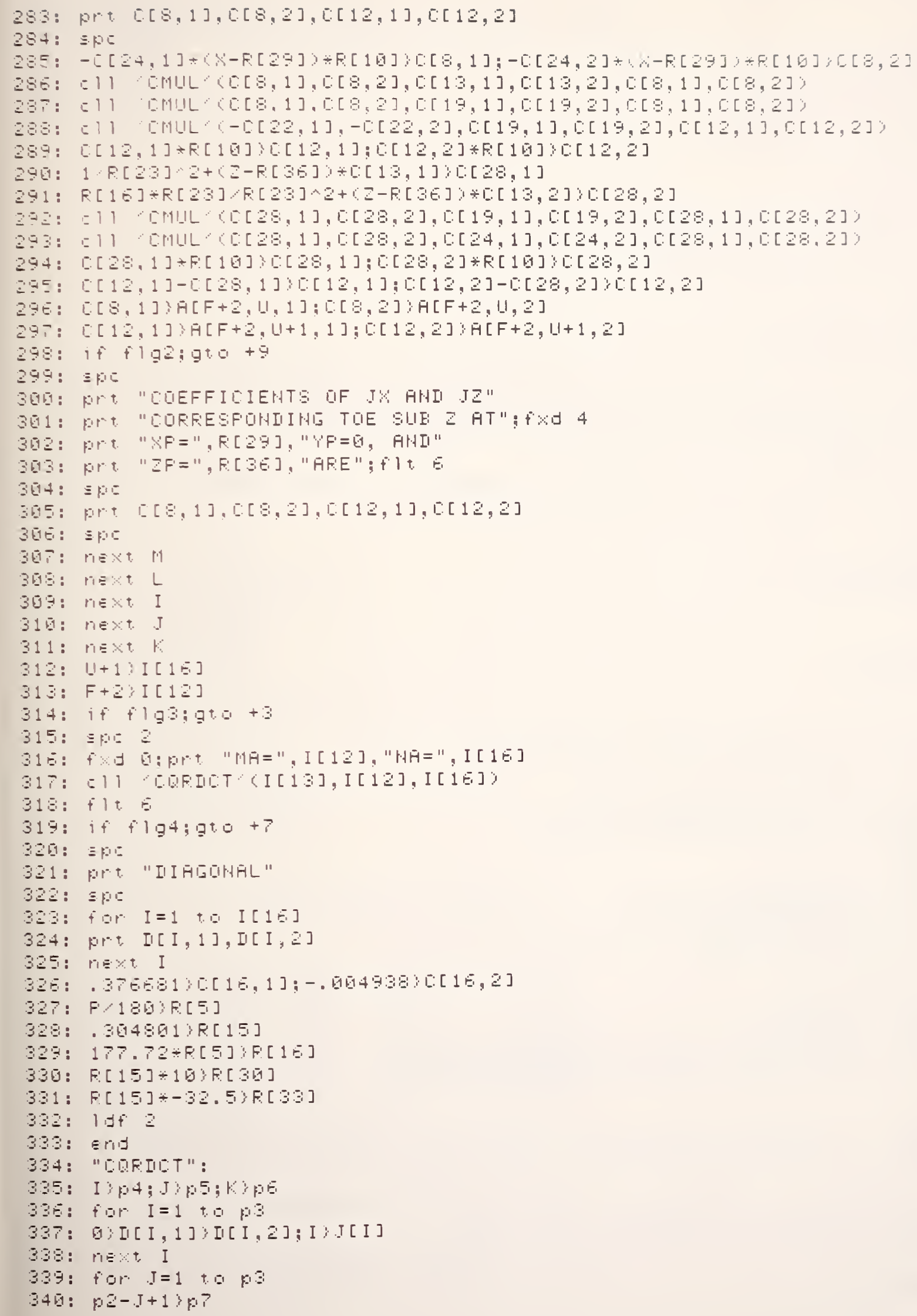




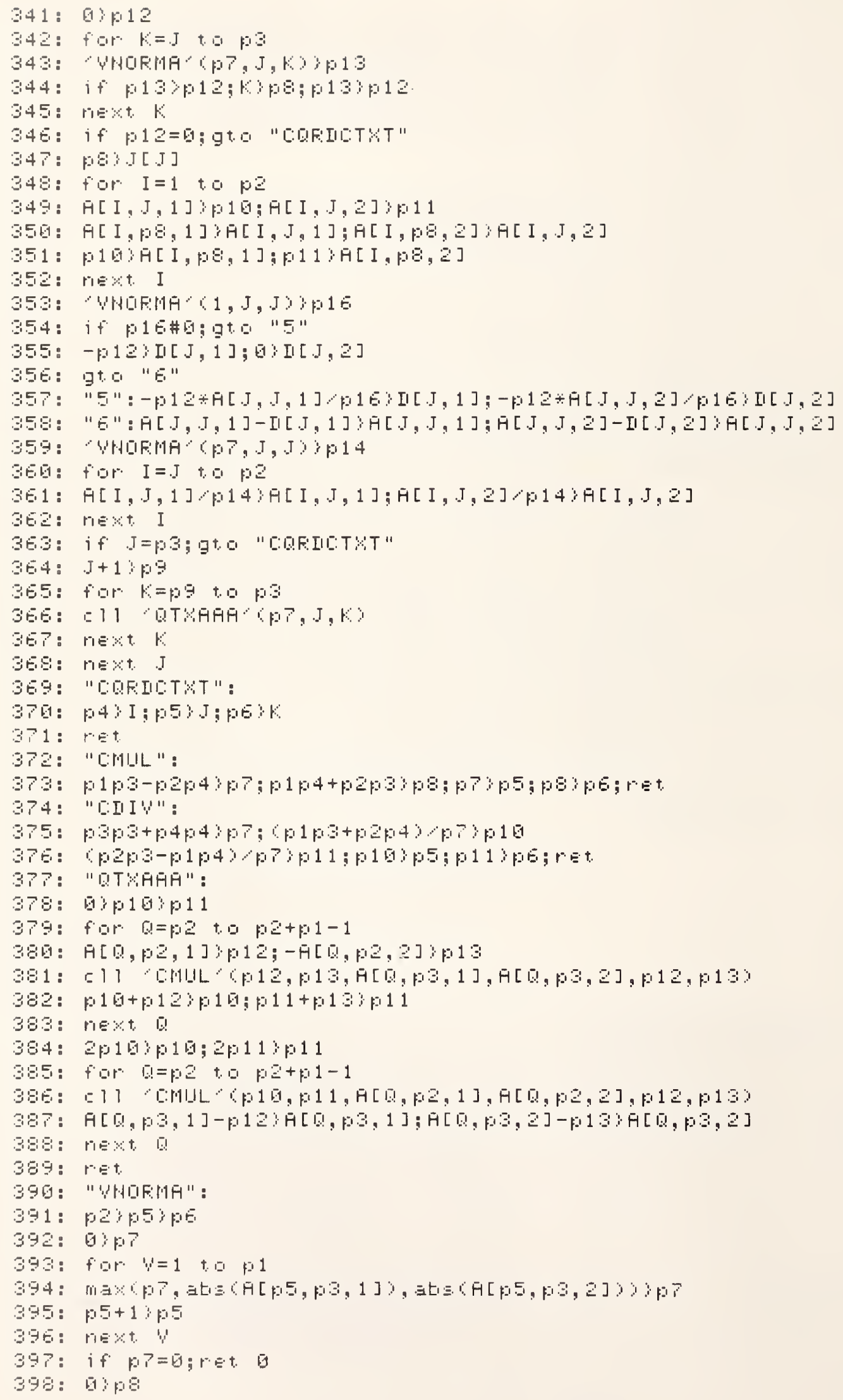


399: for $\psi=1$ to $k 1$

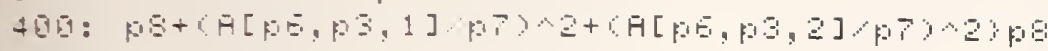

4. 1: pet+1 poe

$402:$ next

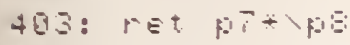

$+159$

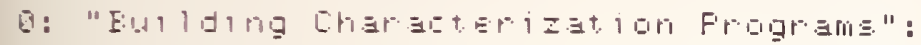

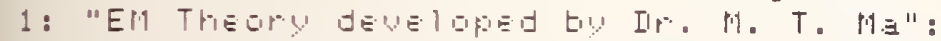

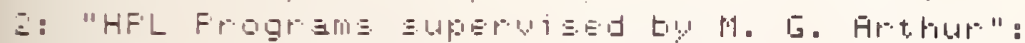

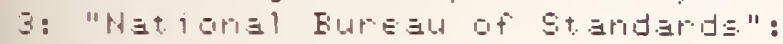

4: "E⿱一兀)

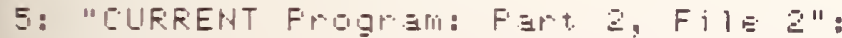

E: $r^{2} \equiv 0$

i: $24 j \cdots+1$

S: $-2>F$

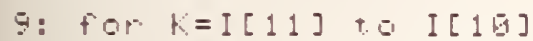

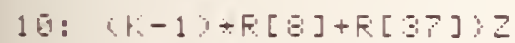

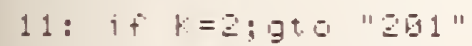

$12: .75-F[15]: F:[5]$

13: $E[+F:[5] ; F[:[]$

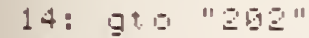

15: "2G1": 2. $25+F[15]$ F [ [5]

1E: $9 \%+F[5] Y F[2:]$

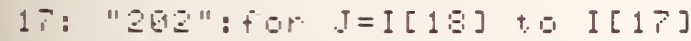

$1 \varepsilon:\langle J-1\rangle+F[\overline{7}]+F[5]\} ' r$

19: for I $=I[15]$ t $[1$ I $[14]$

20: $\quad I-1\rangle+F[E]+F[1] ;$

21: $F+3 ; F$

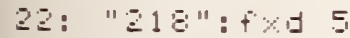

$23: \leq p 0:$

24: \%०t. "El FIELII IIHTH HTFIELII FIIIHTS:"

25: 350

2E: "EHTEF:" :

27:

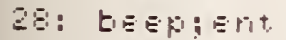

25

36

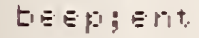

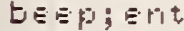

$31:$

32

LEefo; Erit

"EHTEF: HWF'\%. AHF

"ENTEF: FHSU,

$\mathrm{F}^{\prime} \mathrm{H}: \mathrm{H}=?^{\prime \prime}, \mathrm{F}[19]$

"EHTEF: HHF'"

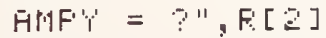

"EHTEF: FHS\%。 FHS

3: "FFIHT EHTFIES":

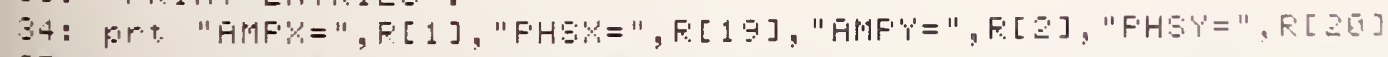

35: prt "HWFZ=",F[S], "FHEZ=",F:[Z1]

$36: 5 F-2$

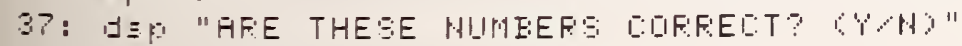

38: Ent." "W

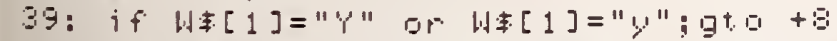

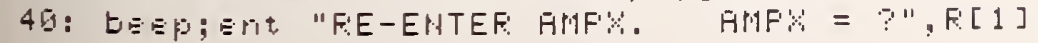




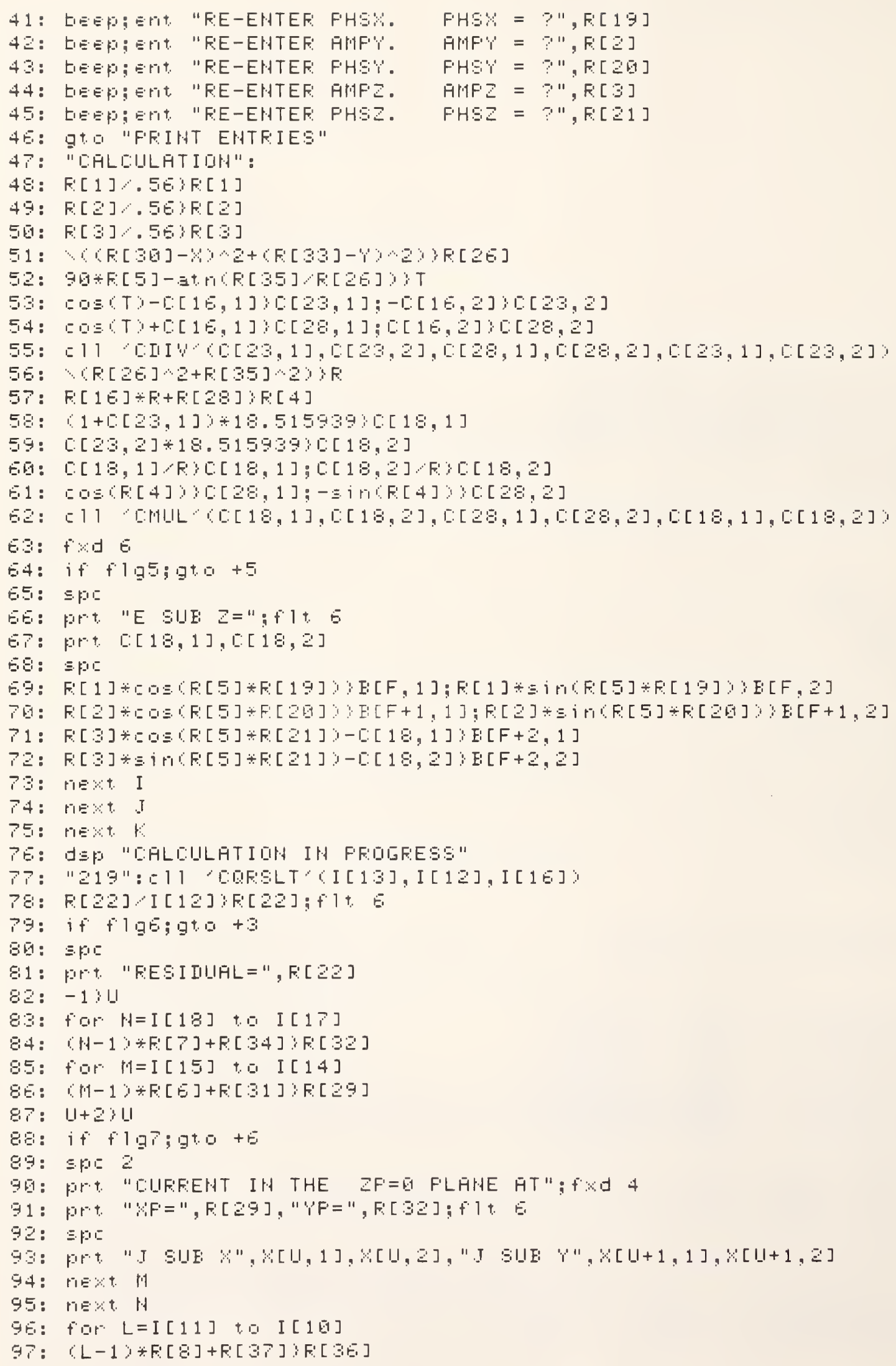




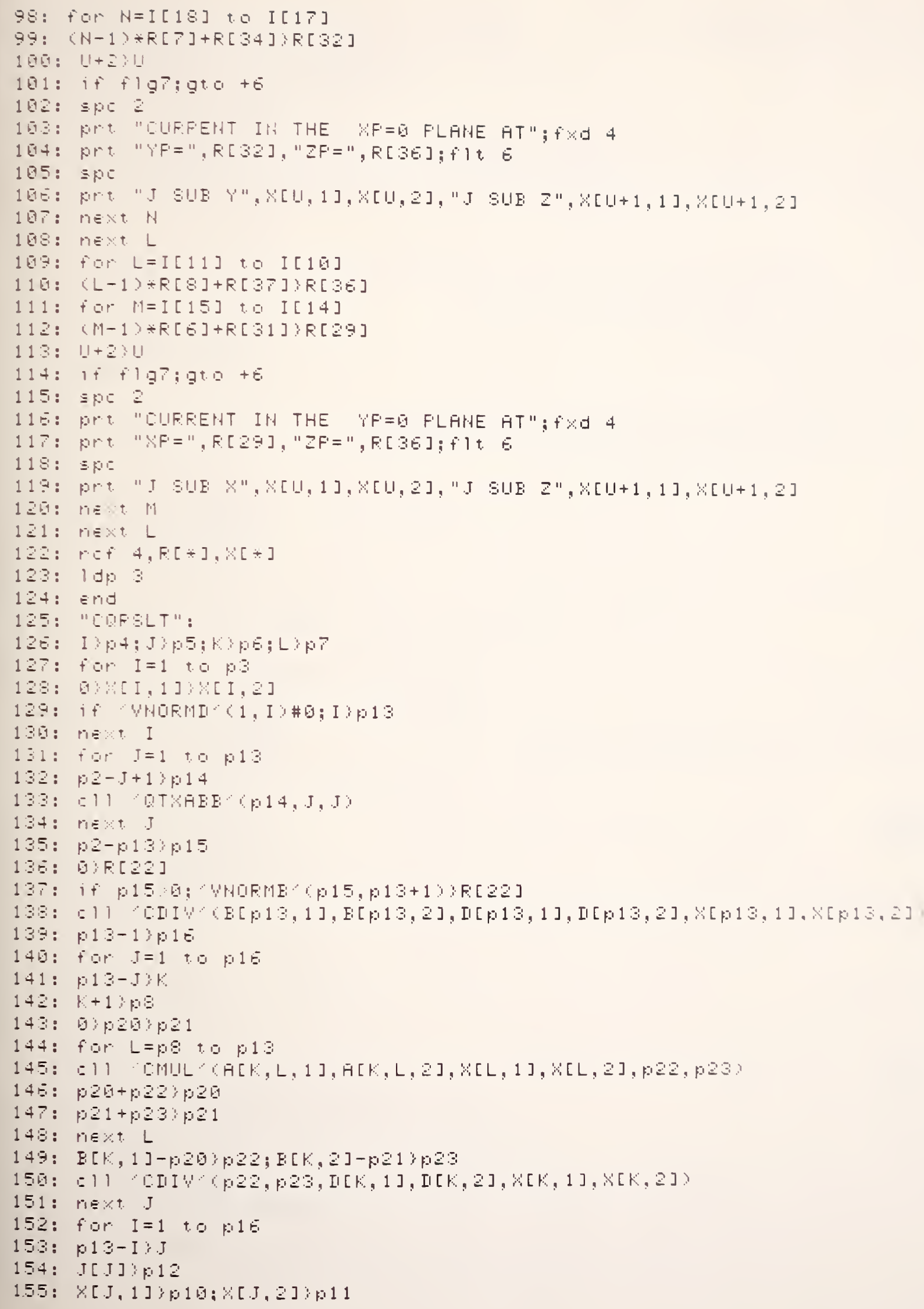




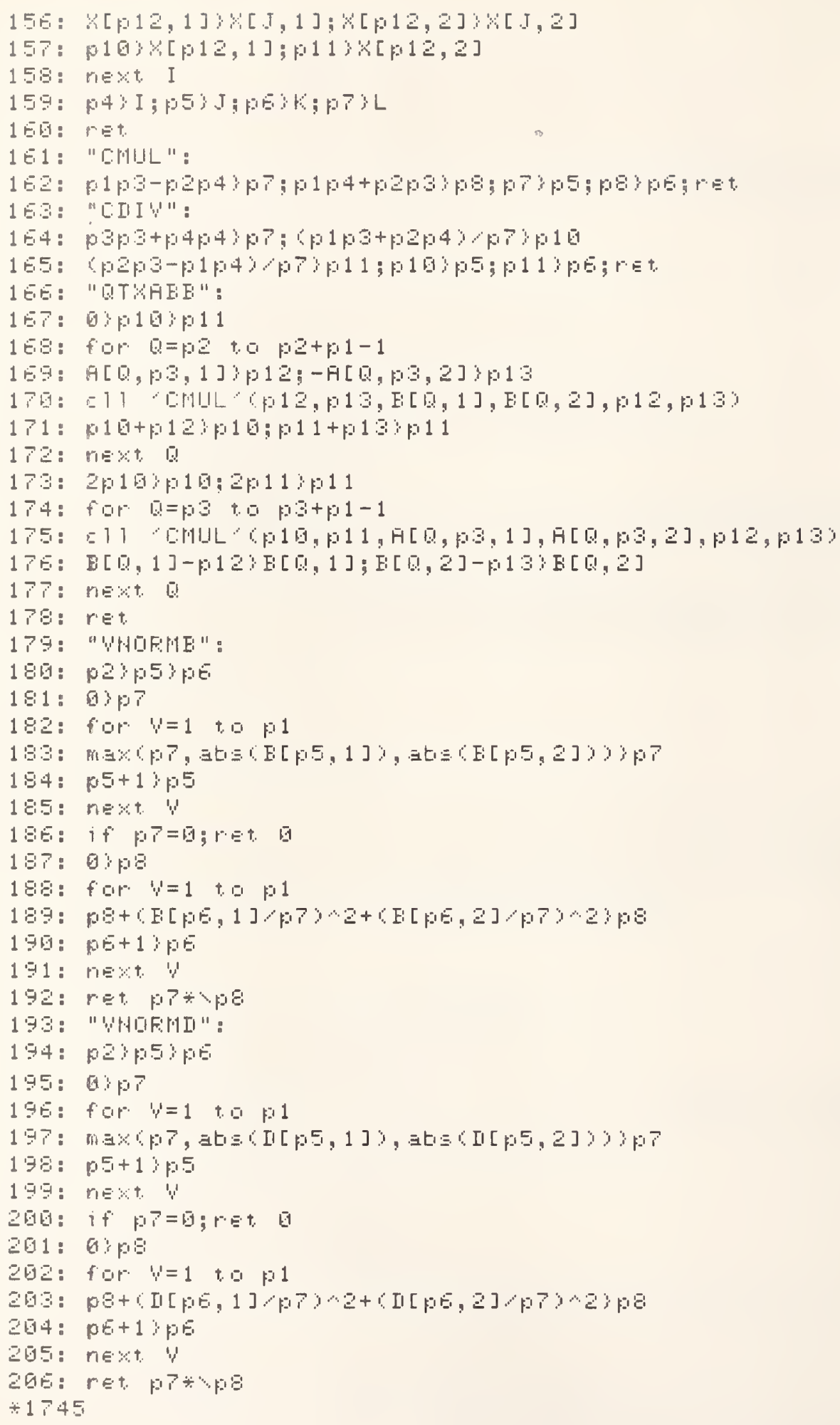




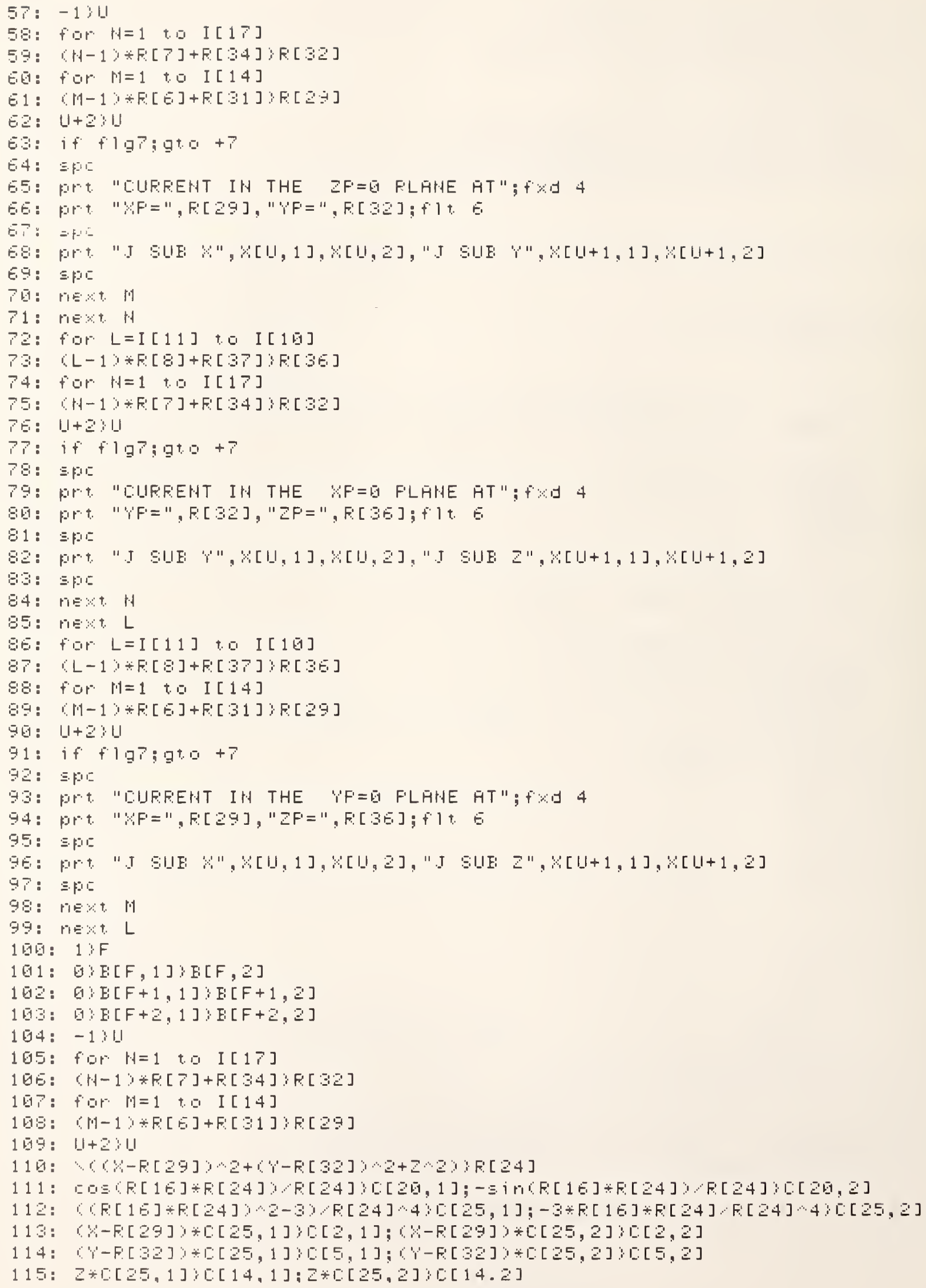




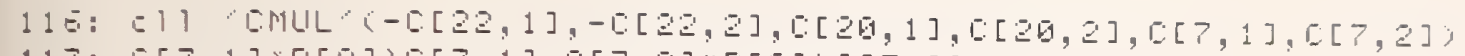

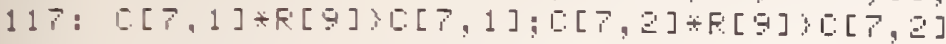

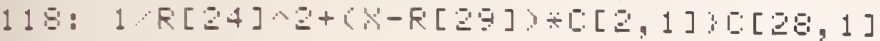

119: $R[1 E] * R[24] R[24] 2+(X-R[29])+[2,2])[2 \Omega, 2]$

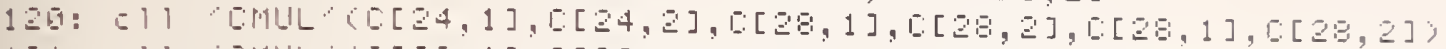

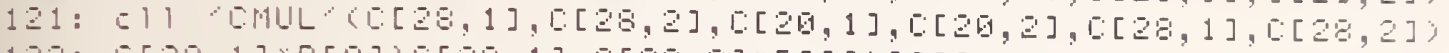

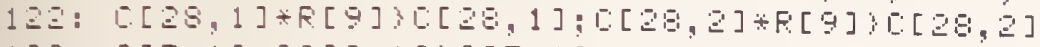

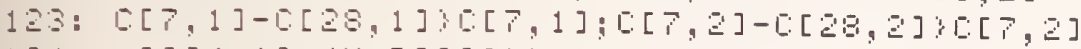

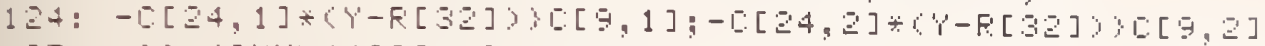

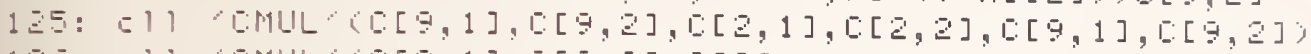

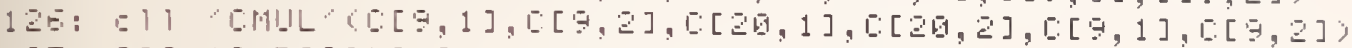

$127:[[9,1] * R[9])[\because, 1],[\because 9,2] \div F[9] ;[: 9,2]$

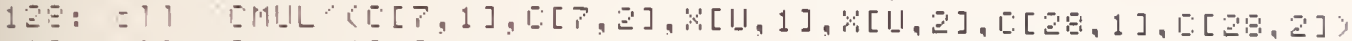

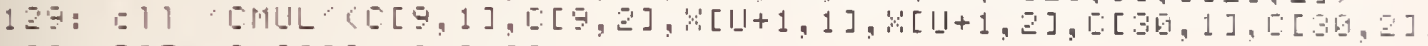

$13[1: E[F, 1]+[:[2], 1]+[:[G, 1]\} E[F, 1]$

$131: E[F, 2]+[[28,2]+[:[B, 2] \mathrm{E}[F, 2]$

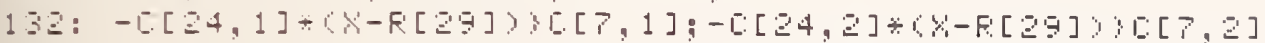

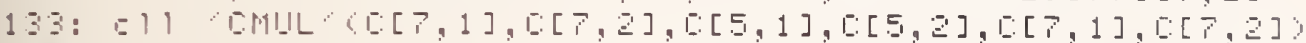

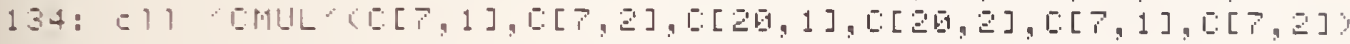

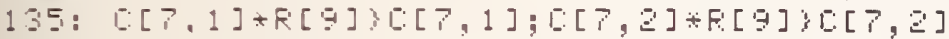

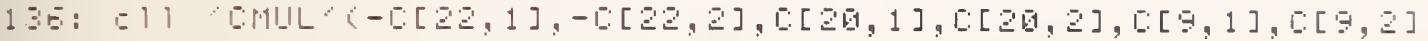

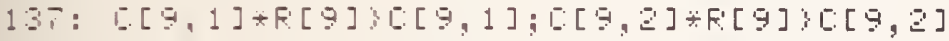

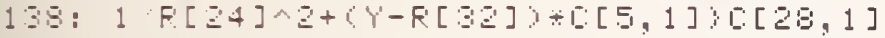

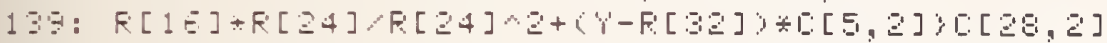

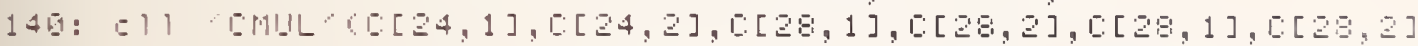

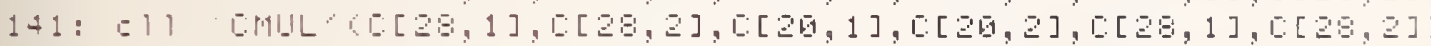

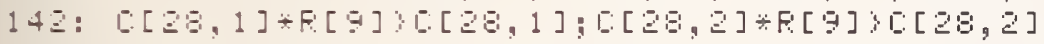

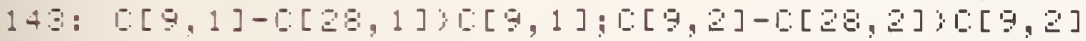

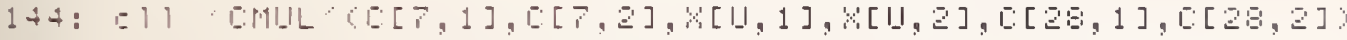

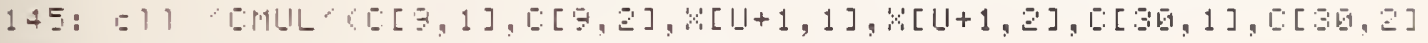

$14 E: \quad E[F+1,1]+[[2,1]+[[3[1,1]: E[F+1,1]$

$14^{-}: \quad E[F+1,2]+[[2 \theta, 2]+[[3], 2]: E[F+1,2]$

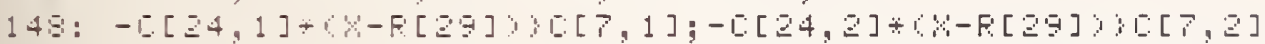

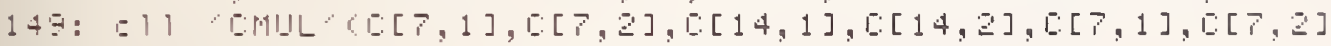

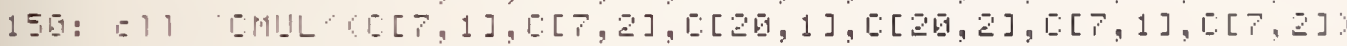

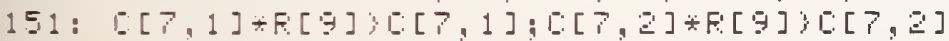

$152:-[[24,1] \div(Y-F[3] ;)[9,1] ;-[[24,2] \div(Y-F[3]\},[9,2]$

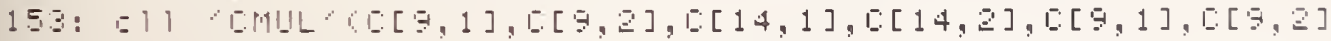

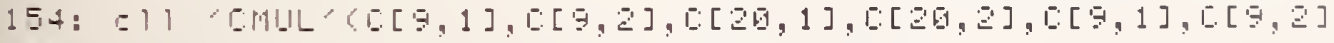

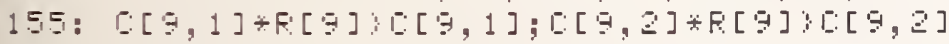

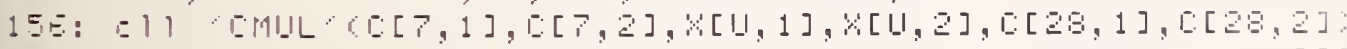

$157:-11$ : [

$158: E[F+2,1]+[[2], 1]+[[30,1] E[F+2,1]$

$159: \quad E[F+2,2]+[:[2,2,2]+[[36,2]) E[F+2,2]$

1E: $\quad r E x+$. M

1E1: next it

1E: for $L=I\left[\begin{array}{lllll}1 & 1\end{array}\right]$ to $I\left[\begin{array}{ll}1 \\ \text { G }\end{array}\right]$

163: $(L-1) * F[B]+F[37]) F[3]$

164: for $H=1$ to I [ 17$]$

1E: $(H-1)+F[P]+F[34] P[32]$

$18: 21+2 ; 1)$

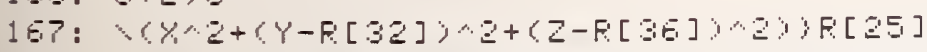

168: G

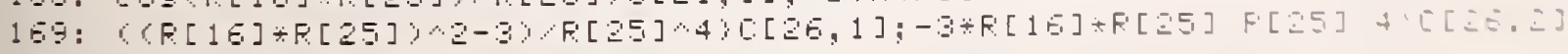

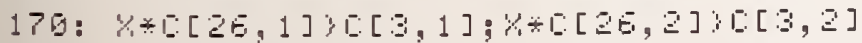

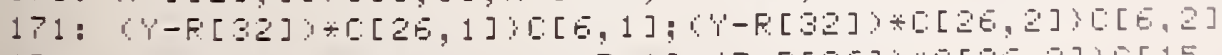

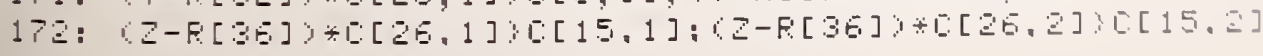


$173:-[[24,1] *(Y-R[3])[[1], 1],-[24,2] \div(Y-R[3])[[1], 2]$

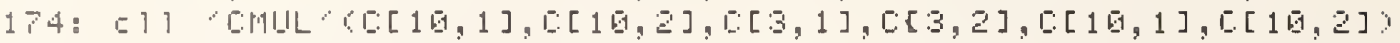

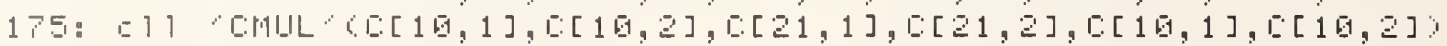

$17 E:[[1], 1] * F[11] ;[[16,1] ;[[10,2] * R[11] ; C[16,2]$

$177:-[[24,1] \div(Z-F[36])[[11,1] ;-[:[2,2] \div(2-F[3 \in])\}[11,2]$

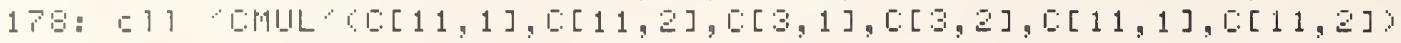

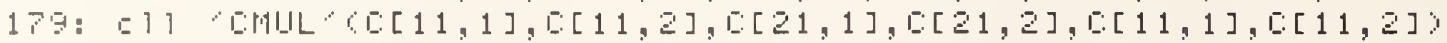

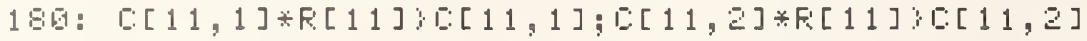

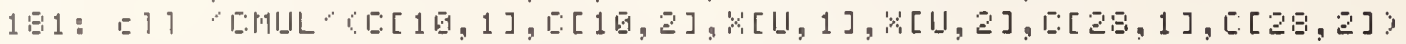

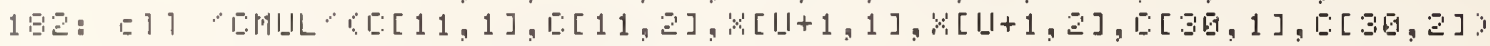

$183: \quad E[F, 1]+[[2,1]+[:[6,1]) E[F, 1]$

$184: E[F, 2]+[:[2,2]+[:[3,2]) E[F, 2]$

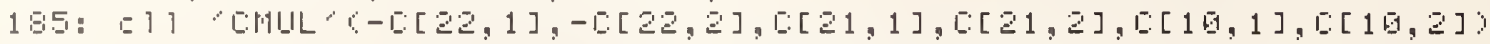

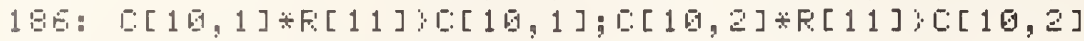

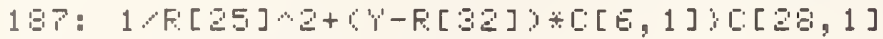

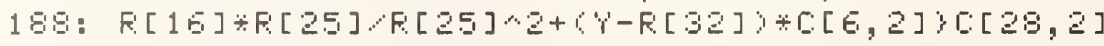

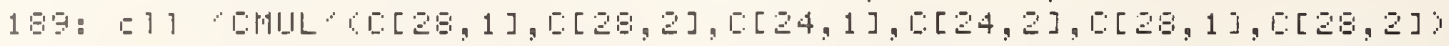

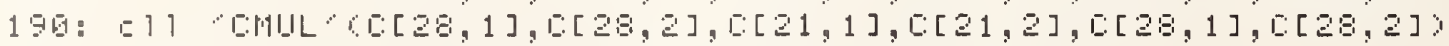

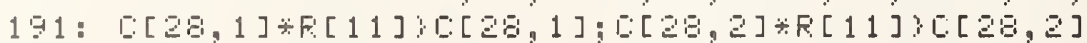

$192:[[10,1]-[:[2,1] ;[16,1] ;[[1,2]-[[25,2] ;[[10,2]$

$193:-[[24,1] \div(2-F[3 E])[[11,1] ;-[24,2] *(2-F[3 \in])[11,2]$

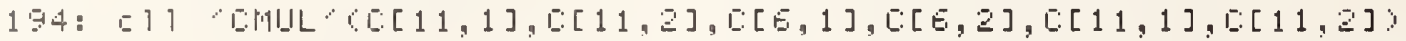

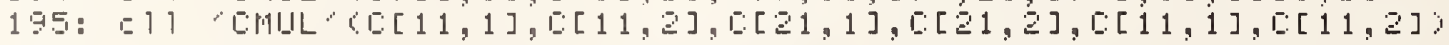

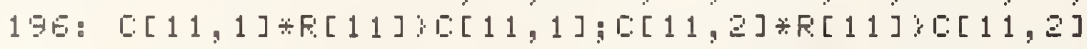

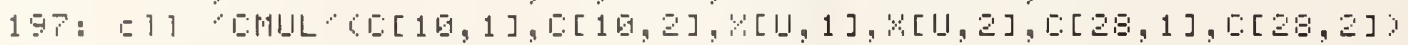

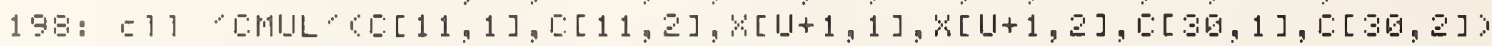

$199: \quad E[F+1,1]+[[2 B, 1]+[[36,1]: E[F+1,1]$

2616: $E[F+1,2]+[[2 G, 2]+[[36,2]: E[F+1,2]$

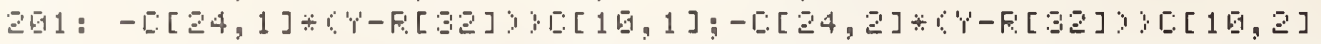

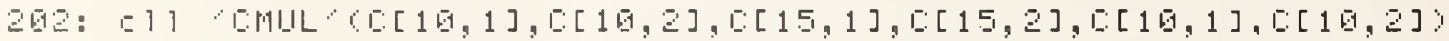

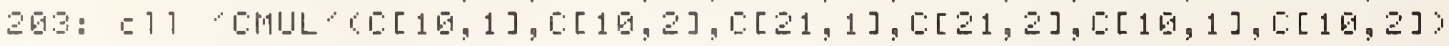

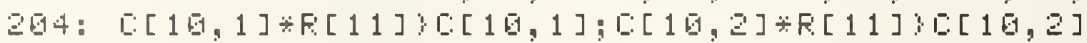

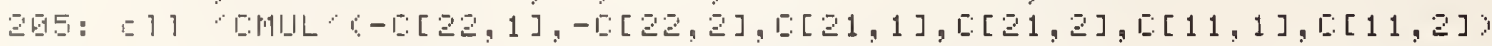

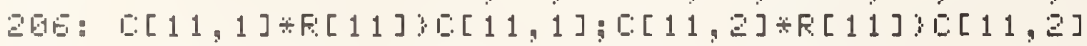

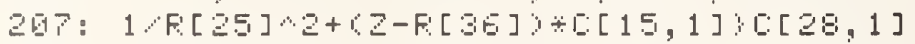

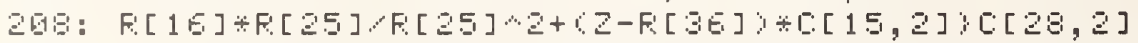

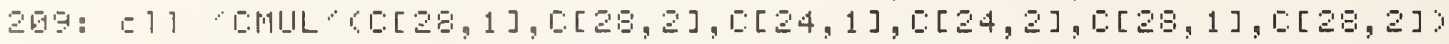

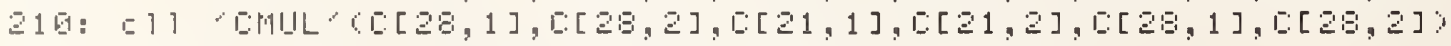

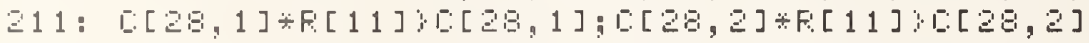

$212:[[11,1]-[:[25,1],[:[1,1] ;[[11,2]-[:[2,2],[[11,2]$

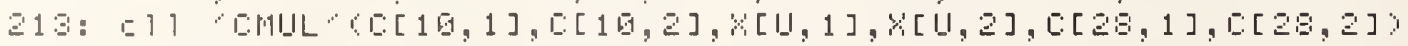

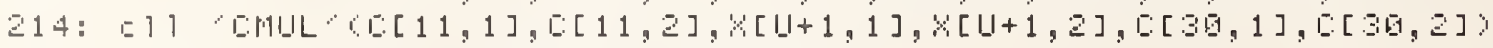

$215: \quad E[F+2,1]+[[28,1]+[[3[1,1]: E[F+2,1]$

$21 E: \quad E[F+2,2]+[[2], 2]+[[3[1,2]: E[F+2,2]$

217: next, H

21日: rext. L

219: for $L=I\left[\begin{array}{llll}1 & 1\end{array}\right]$ to $I[16]$

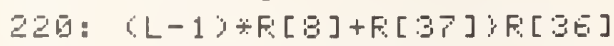

221: for $M=1$ t. a I [ 14]

22Z: (H-1)*R[G]+R[31])R[2]]

$223: 1 \mathrm{I}+2,11$

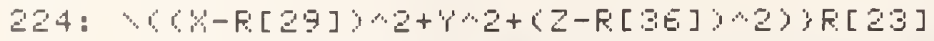

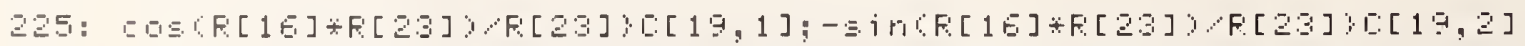

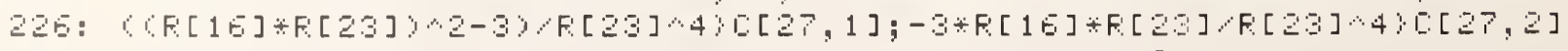

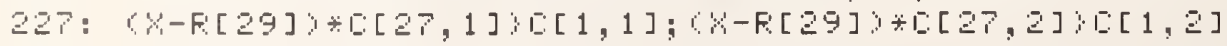

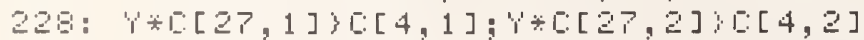

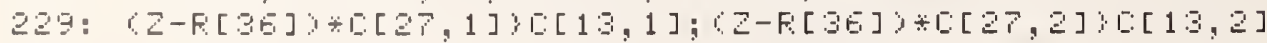

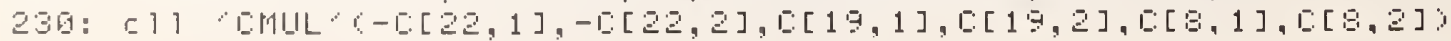




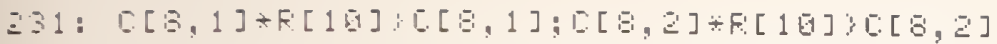

$232: 1$ R $[23] \cdots+(X-R[29]) *[1,1] ;[28,1]$

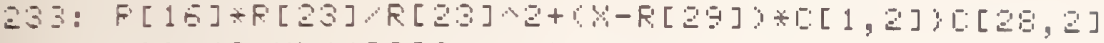

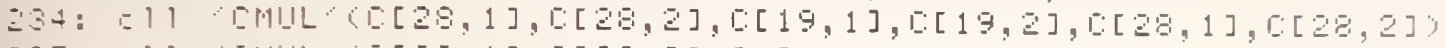

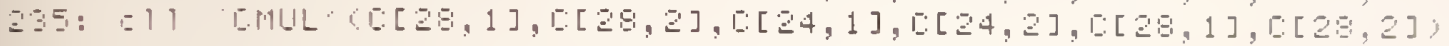

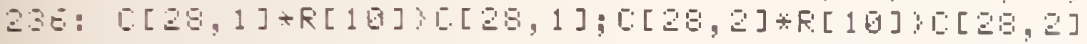

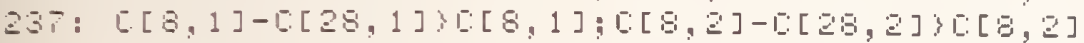

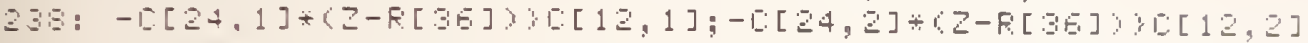

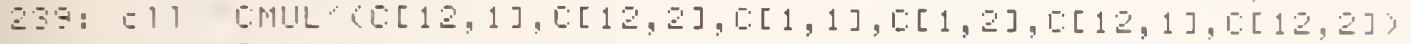

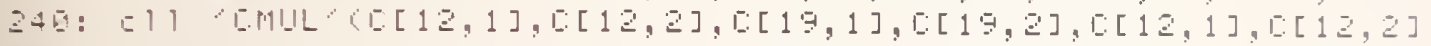

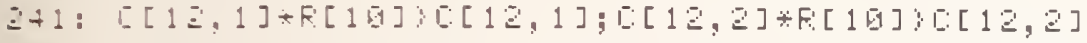

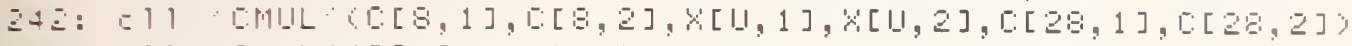

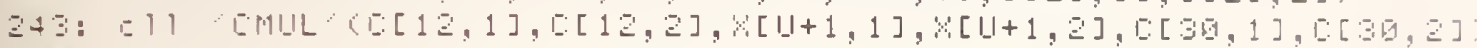

$3+4: E[F, 1]+[[28,1]+[[36,1]) E[F, 1]$

$2+5: E[F, Z]+[[2 \theta, 2]+[: 36,2]\} E[F, 2]$

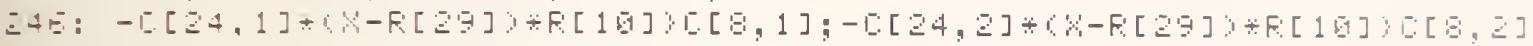

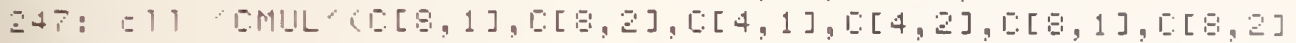

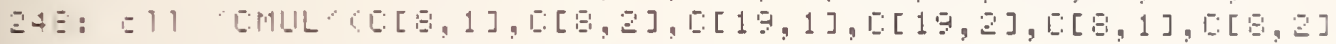

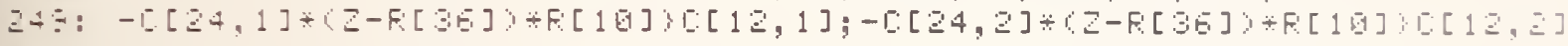

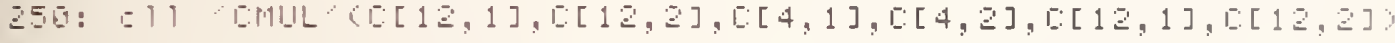

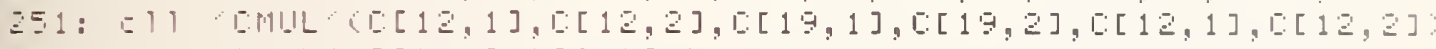

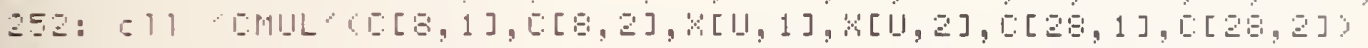

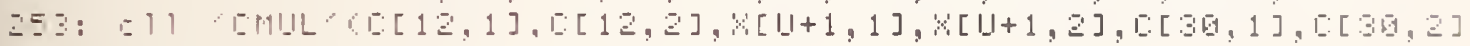

$25+: E[F+1,1]+[[2,1]+[[3,1], E[F+1,1]$

$25: E[F+1,2]+[:[2,2]+[:[3,2] B[F+1,2]$

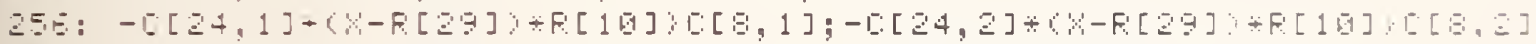

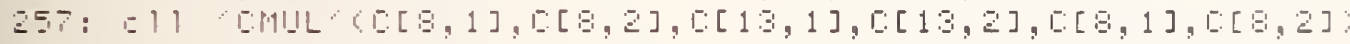

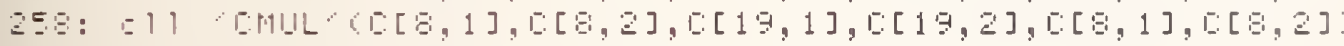

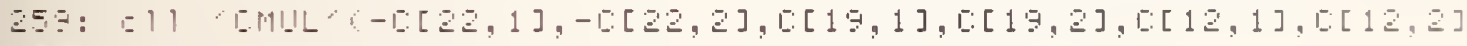

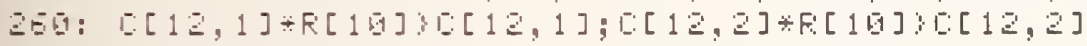

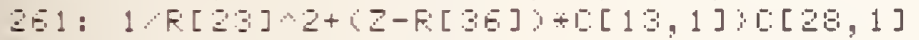

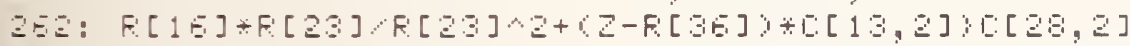

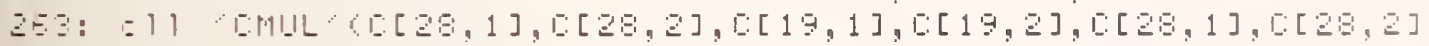

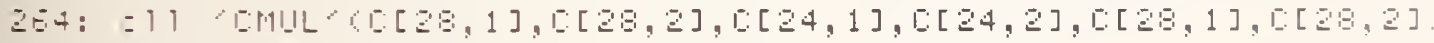

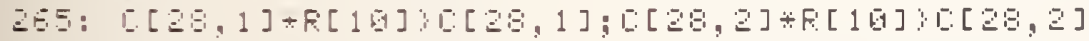

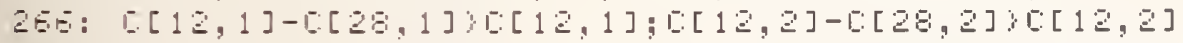

2E- : $: 11$ [

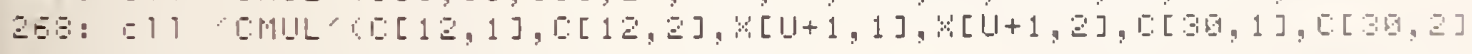

$2 E: E[F+2,1]+[[2], 1]+[[3,1]:[F+2,1]$

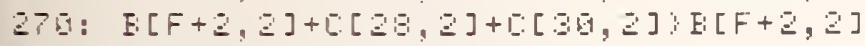

Z1: nExt in

272: nEXt, L

$273: U+1) I[1]]$

274: $F+2 ;[112]$

275: $1 f$ flg $19 t 0+z$

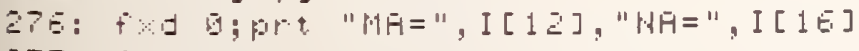

27?: $+\times d E$

27E: . $37 E 61 ; 0[16,1] ;-.604936 ;[:[1 E, 2]$

27:: F/1EG\}F[5]

280: . 304801\}R[15]

281: $177.72 * R[5]\} R[1 E]$

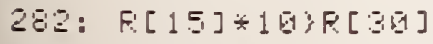

$283: F[15] \div-32.5 ; f[33]$

284: 350

285: PH. "E SUE $\%$ E EIIE "YHAII E SUE 2 AT THE FIELI FUINT"

28E: $2-4 \div 8:[15]\} F[5]$

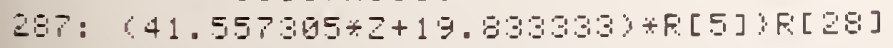

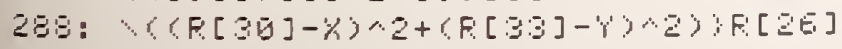




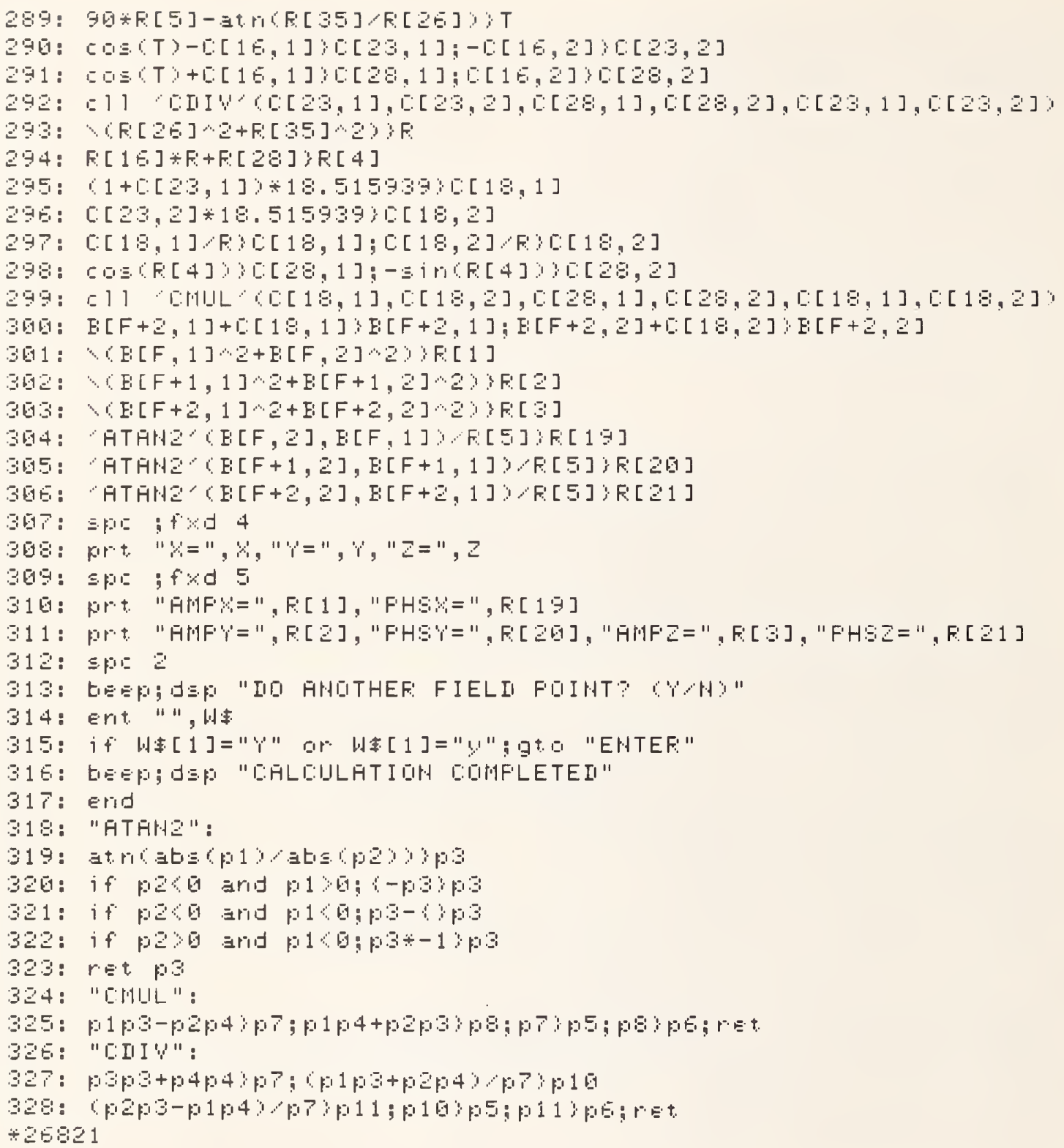




\section{B.2 Variable Assignments}

Complex Variable Assignments

The complex variables are stored in arrays with one extra dimension.

Thus the complex variable can be stored in two parts: the real part, stores in the top $(X[X, 1])$, and the imaginary part, stored in the bottom $(X[X, 2])$.

All complex arrays are assigned their own array variable while the rest of the complex variables are assigned in the array variable "C".

\begin{tabular}{|c|c|}
\hline FORTRAN VARIABLE & HPL VARIABLE \\
\hline$A(M, N)$ & $A[M, N, 2]$ \\
\hline$B(M)$ & $B[M, 2]$ \\
\hline $\operatorname{DIAG}(N)$ & $D[N, 2]$ \\
\hline$X S(N)$ & $X[N, 2]$ \\
\hline & $C[50,2]$ pool of complex variables \\
\hline AML3 & $C[1]$ \\
\hline AMN1 & $C[2]$ \\
\hline ANL2 & $C[3]$ \\
\hline BML 3 & $C[4]$ \\
\hline BMN1 & $C[5]$ \\
\hline BNL2 & $C[6]$ \\
\hline $\operatorname{cJ} \times 1$ & $C[7]$ \\
\hline $\cos \times 3$ & $C[8]$ \\
\hline CJV1 & $C[9]$ \\
\hline CJY2 & $C[10]$ \\
\hline CJZ2 & $C[11]$ \\
\hline $\operatorname{cuZ3}$ & $C[12]$ \\
\hline CML 3 & $C[13]$ \\
\hline CMN1 & $C[14]$ \\
\hline CNL2 & $C[15]$ \\
\hline $\mathrm{Cl}$ & $C[16]$ \\
\hline$c 2$ & $C[17]$ \\
\hline ESUBZ & $C[18]$ \\
\hline
\end{tabular}




$\begin{array}{ll}\text { GML3 } & C[19] \\ \text { GMN1 } & C[20] \\ \text { GNL2 } & C[21] \\ \text { OMS4P } & C[22] \\ \text { RSUBV } & C[23] \\ \text { SO4PE } & C[24] \\ \text { S1 } & C[25] \\ \text { S2 } & C[26] \\ \text { S3 } & C[27] \\ \text { TEMP } & C[28] \\ \text { TEMP } & C[29] \\ \text { TEMP } & C[30]\end{array}$




\section{Real Variable Assignments}

The most commonly used real variables are assigned simple variables and the rest of the real variables are assigned in the variable array "R".

\begin{tabular}{c} 
FORTRAN VARIABLE \\
\hline PI \\
R \\
THETA \\
$X$ \\
$Y$ \\
$Z$
\end{tabular}

A.MPX

AMPY

AMPZ

$A R G$

DTOR

$\mathrm{DX}$

DY

$D Z$

DXDY

$D \times D Z$

DYDZ

EPS

ESUBZ

FREQ

FTOM

KAY

MU

OMEGA

PHSX

PHSY

PHSZ

RESID

RIIL3

RMN1
HPL VAR I ABLE

$P$

$R$

$T$

$x$

$Y$

Z

$R[60]$ pool of real variables

$\mathrm{R}[1]$

$\mathrm{R}$ [2]

$\mathrm{R}$ [3]

$\mathrm{R}$ [4]

$\mathrm{R}$ [5]

$\mathrm{R}$ [6]

$\mathrm{R}$ [7]

$\mathrm{R}$ [8]

$\mathrm{R}$ [9]

$\mathrm{R}$ [10]

$R$ [11]

$R$ [12]

$\mathrm{R}$ [13]

$\mathrm{R}$ [14]

$\mathrm{R}$ [15]

$\mathrm{R}$ [16]

$R$ [17]

$\mathrm{R}$ [18]

$\mathrm{R}$ [19]

$\mathrm{R}$ [20]

$\mathrm{R}$ [21]

$\mathrm{R}$ [22]

$\mathrm{R}$ [23]

$\mathrm{R}$ [24] 


$\begin{array}{ll}\text { RNL2 } & R[25] \\ \text { RSUBT } & R[26] \\ \text { TC } & R[28] \\ \text { XP } & R[29] \\ \text { XPP } & R[30] \\ \text { XSTART } & R[31] \\ \text { YP } & R[32] \\ \text { YPP } & R[33] \\ \text { YSTART } & R[34] \\ \text { ZD } & R[35] \\ \text { ZP } & R[36] \\ \text { ZSTART } & R[37] \\ \text { X2 } & R[38] \\ \text { Y2. } & R[39] \\ \text { Z2 } & R[40]\end{array}$


Integer Variable Assignments

The most commonly used integer variables, and variables used in "for" loops, are assigned simple variables. The integer array "JPVT" is stored in its own variable array "J" while the rest of the integer variables are stored in the array "I".

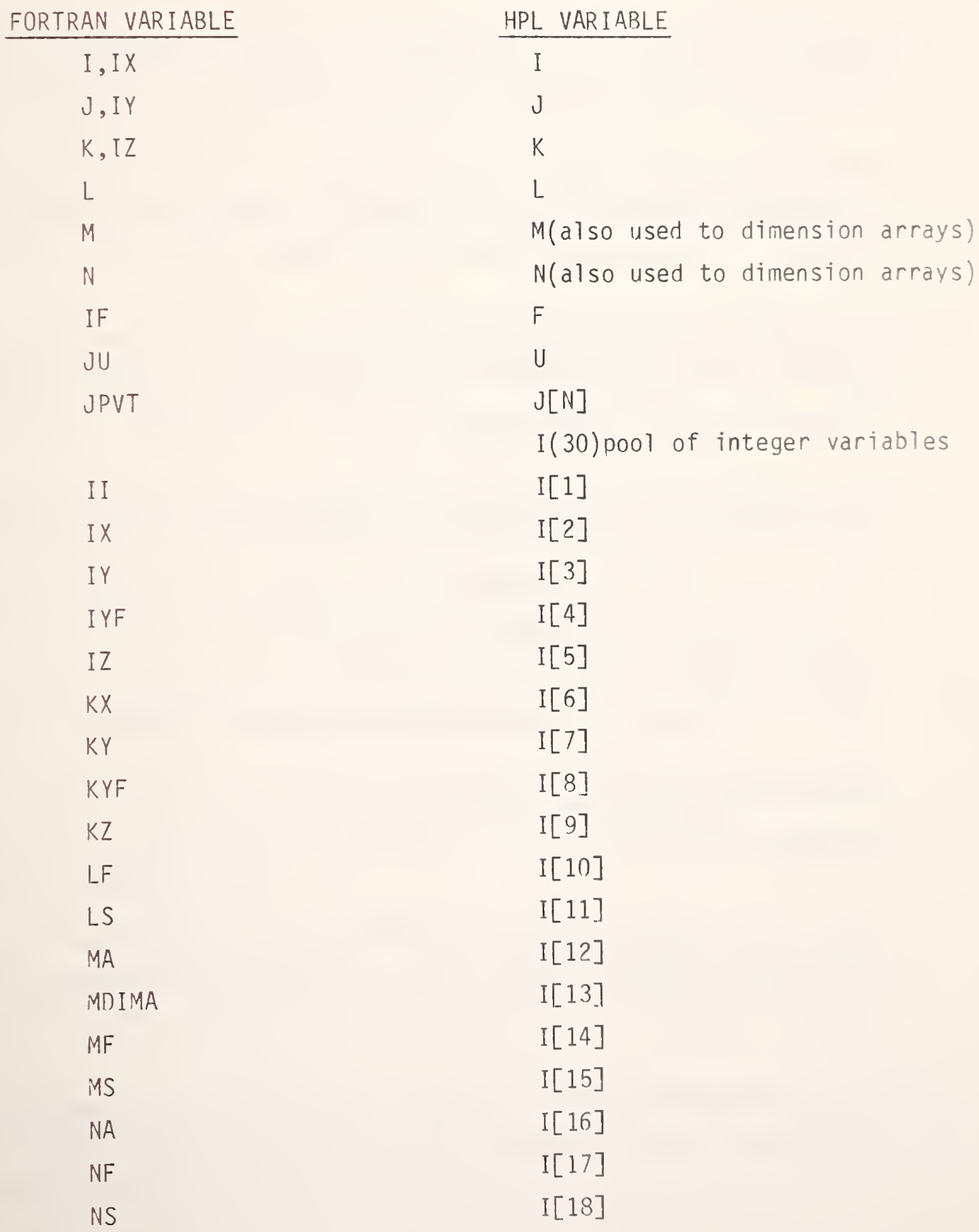




\section{B.3 Instructions for Running HPL Programs}

To run the HPL CURRENT program, follow this procedure:

1. With the power to the computer turned on, insert the magnetic program tape in accordance with the computer's operating instructions.

2. Press LOAD and type in 1, or type $1 \mathrm{df} 1$, and press EXECUTE. This will load CURRENT program, Part 1, into the computer.

3. Make any program changes, such as flags, as needed or desired. These are discussed in section 6.3. The procedure listed here is based on no changes to the program as shown in section B. 1 .

4. Press RUN. This will cause the computer to print the heading "CURRENT PROGRAM" on the paper tape. Next, the computer wil1 print "COORDINATES OF FIELD POINTS:" on the tape.

5. The computer will beep and display "ENTER X1. X1 = ?" as a prompt.

6. Type in the first $X$-coordinate value and press CONTINUE. The computer will beep and display "ENTER X2. $x 2=$ ?."

7. Type in the second X-coordinate value and press CONTINUE.

8. Continue in this marner until X1, X2, Y1, Y2, Z1, and $Z 2$ have a 11 been entered.

9. After $Z 2$ has been entered, the computer will print out the values of X1 through 2 ? that were entered. Then it will display "ARE THESE NUMBERS CORRECT? $(\mathrm{Y} / \mathrm{N})$."

10. If all are correct, type in either $y$ or $Y$ and press CONTINUE. If any are not correct, type in either $n$ or $N$ and press CONTINUE. 
11. If you have typed $\mathrm{n}$ or $\mathrm{N}$, the computer will beep and display "RE-EMTER X1. X1?" as a prompt. Repeat steps 6 through 10 until the numbers printed out in step 9 are correct.

12. When you type y or $Y$, the computer will display "THIS CALCILATION TAKES $z$ MINUTES." Take a break while the computer does its thing.

13. When the computer completes this calculation of the A coefficients and has performed the QR factorization on the [A] matrix, it automaticaliy loads CURRENT program, Part 2 from file \#2.

14. The computer then prints "EM FIELD DATA AT FIELD POINTS:", heeps, and displays "ENTER AMPX. AMPZ = ?" as a prompt.

15. Type in the amplitude of the $x$-direction component of the measured field at data point (X1, Y1, Z1) and press CONTINUE. The computer will beep and display "ENTER PHSX. PHSX = ?" as a prompt.

16. Type in the phase of the $X$-direction component of the measured field at data point $(X 1, Y 1, Z 1)$ and press CONTINUE. The computer will beep and display "ENTER AMPY. AMPY = ?" as a prompt.

17 Continue in this manner until AMPX, PHSX, AMPY, PHSY, AMPZ, AND PHSZ for data point $(X 1, Y 1, Z 1)$ have been entered.

18. After PHSZ has been entered, the computer will print out the values of AMPX through PHSZ that were entered. Then it will display "ARE THESE NUMBERS CORRECT? ( $Y / N)$."

19. If correct, type in either $y$ or $Y$ and press CONTINUE. If not correct. type in either $\mathrm{n}$ or $\mathrm{N}$ and press CONTINUE.

20. If you have typed $\mathrm{n}$ or $\mathrm{N}$, the computer will beep and display "RE-ENTER AMPX. AMPX $=$ ?" as a prompt. Repeat steps 15 through 19 until the numbers printed out in step 18 are correct. 
21. When you type y or $Y$, the computer again prints, "EM FIELD DATA AT FIELD POINTS:", beeps, and displays "ENTER AMPX. AMPX = ?" as a prompt.

22. Repeat steps 15 through 21 for measured field data at data points (X2, $Y 1, Z 1),(X 1, Y$ Y,$Z 1),(X 2, Y 2, Z 1),(X 1, Y 1, Z 2),(X 2, Y 1, Z 2),(X 1, Y 2$ $\mathrm{Z2})$, and $(X 2, Y 2, Z 2)$ in that order.

23. After the measured field data have all been entered correctly for data point (X2, Y2, Z2) and you have pressed y or Y, the computer will display "CALCULATION IN PROGRESS." This calculation requires several seconds.

24. When the computer completes this calculation of the currents $\mathrm{J}$, it automatically loads and runs the FIELD program stored in file \#3.

To run the HPL FIELD program, follow this procedure:

1. If the FIELD program is to be run immediately following the CURRENT program, the computer will have printed the heading "FIELD PROGRAM" on the paper tape immediately after loading the FIELD program in step 24, above. Procede to step 4, below.

If the field program is to be run at some later time following the running of the CURRENT program, and the computer memory has been erased so that it no longer contains the results of running the CIIRRENT program, begin with step 2, below.

2. With the power to the computer turned on, insert the magnetic program tape in accordance with the computer's operating instructions.

3. Press LOAD and type 3, or type $1 \mathrm{df} 3$, and press EXECUTE. This will load the FIELD program into the computer.

4. Make any program changes as needed or desired. These are discussed in section 6.4. The procedure listed here is based on no changes to the program as shown in section B.1. 
5. Press RUN. This will cause the computer to print the heading "FIELD PROGRAM" on the paper tape. Next, the computer prints "FIELD POINT POSITION:" on the tape.

6. The computer will beep and display "ENTER $X . X=$ ?" as a prompt.

7. Type in the $X$-coordinate of the arbitrary field point at which the field strength is to be computed and press CONTINUE. The computer will beep and display "ENTER $Y$. $Y=$ ?."

8. Type in the $Y$-coordinate of the arbitrary field point and press CONTINUE. The computer will print out the values of $X, Y$, and $Z$ that were entered. Then it will display "ARE THESE NUMBERS CORRECT? $(\mathrm{Y} / \mathrm{N})$."

10. If correct, type in either $y$ or $Y$ and press CONTINUE. If not correct, type in either $\mathrm{n}$ or $\mathrm{N}$ and press CONTINUE.

11. If you have typed $\mathrm{n}$ or $\mathrm{N}$, the computer will beep and display "RE-ENTER $X . \quad X=$ ?" as a prompt. Repeat steps 7 through 10 until the numbers printed out in step 9 are correct.

12. When you type y or $Y$, the computer will display "CALCULATION IN PROGRESS." This calculation requires several seconds.

13. When the computer completes this calculation of the field at point ( $X$. $Y$. $Z$ ), it prints "E SUB $X, E$ SUB $Y$, and $E$ SUB $Z$ at the FIELD POINT." Then it prints out the values of $X, Y, Z$, AMPX, PHSX, AMPY, PHSY, AMPZ, AND PHSZ.

14. Then the computer displays "DO ANOTHER FIELD POINT? ( $Y / N)$."

15. If yes, type in either $y$ or $Y$ and press CONTINUE. If no, type in either $n$ or $N$ and press continue.

16. If you have tyoed $y$ or $Y$, the computer will print "FIELD POINT PUSITIU\%:" on the tape, and continue from step 6 , above. If you have typed 17 or $\because$, the computer displays "CALCULATION COMPLETED" and ends the run. 


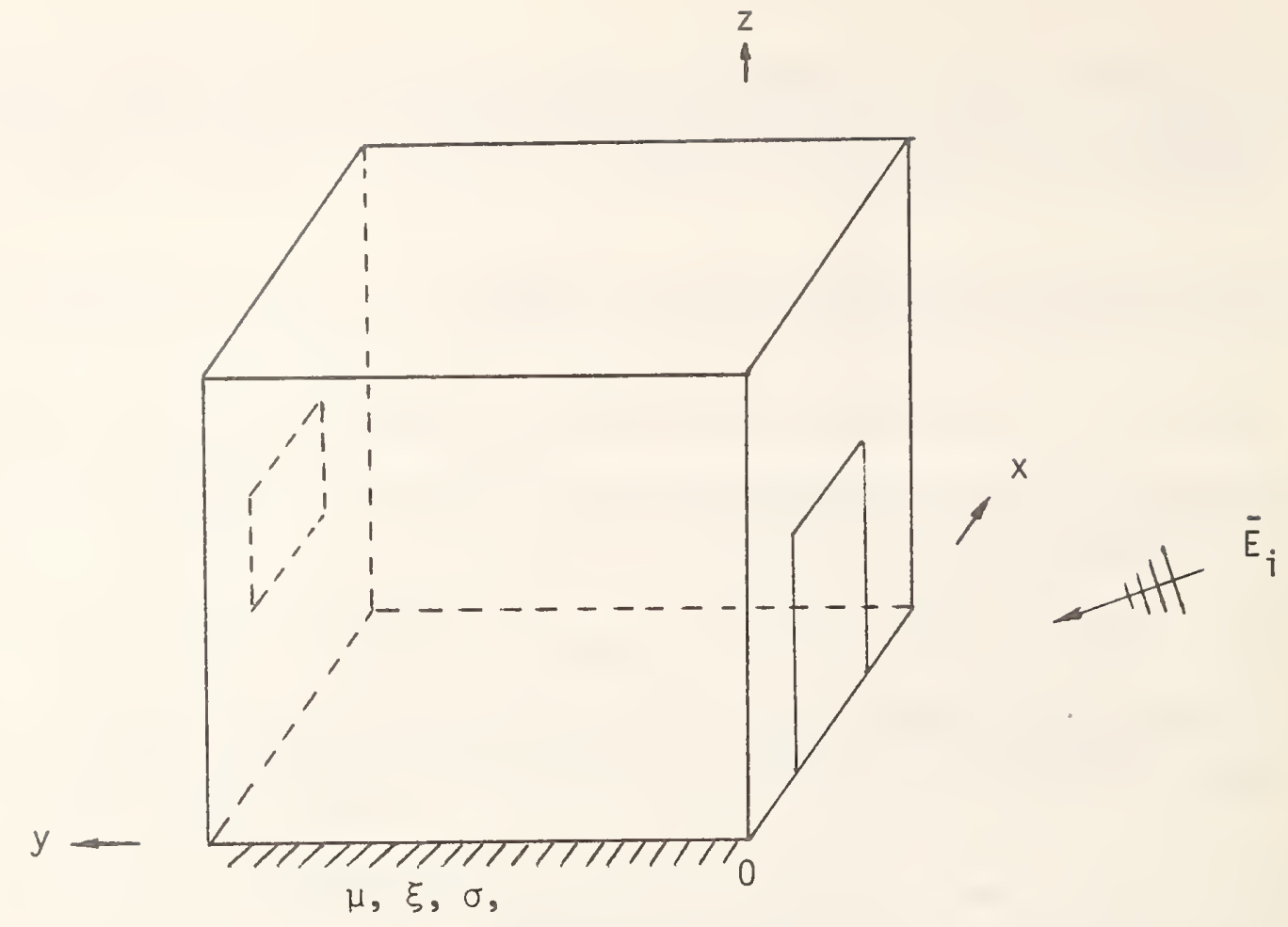

Figure 1. A theoretical model of penetration of electromagnetic waves into a cavity with apertures.

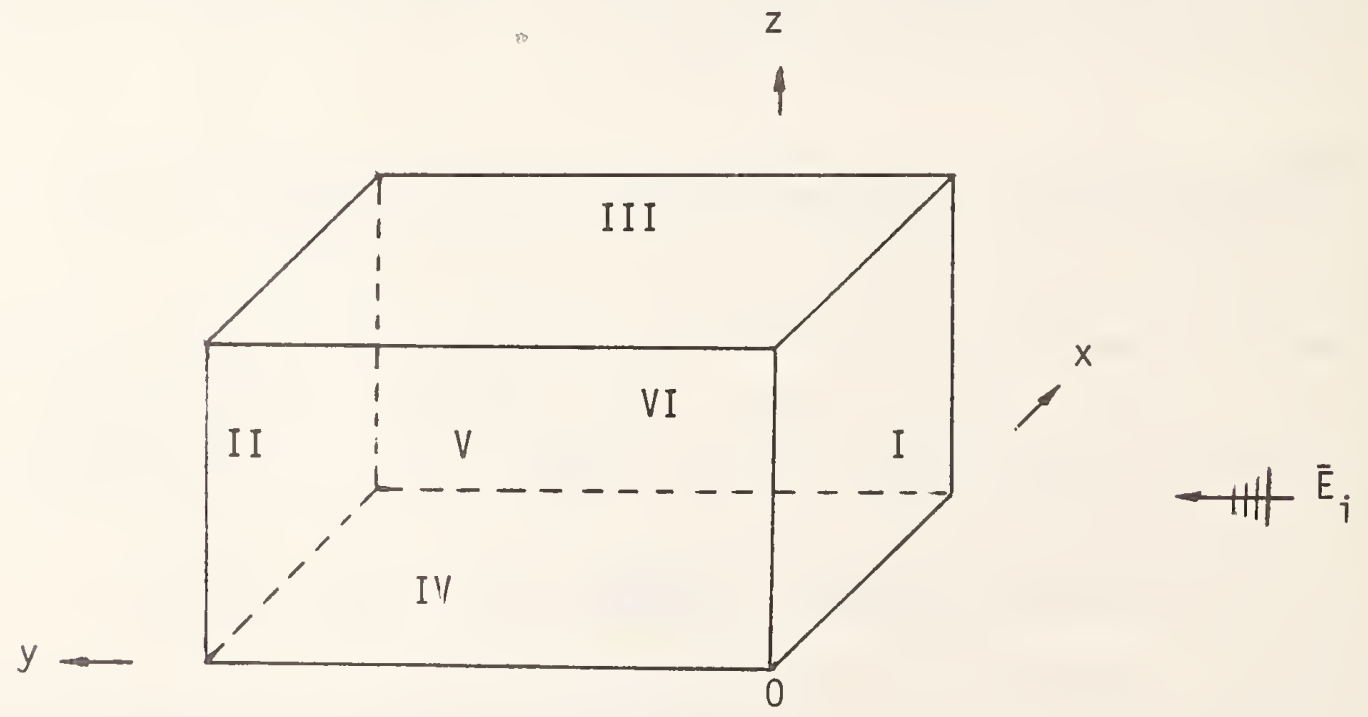

Figure 2. A solid perfectly conducting box without apertures in free space. 


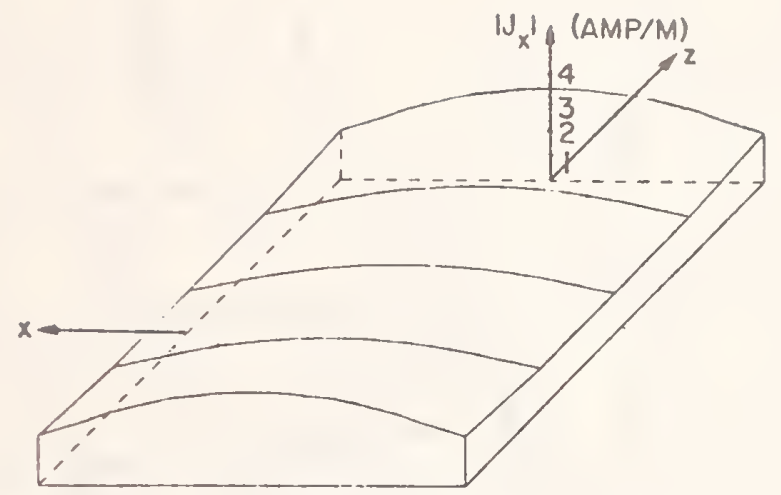

(3)

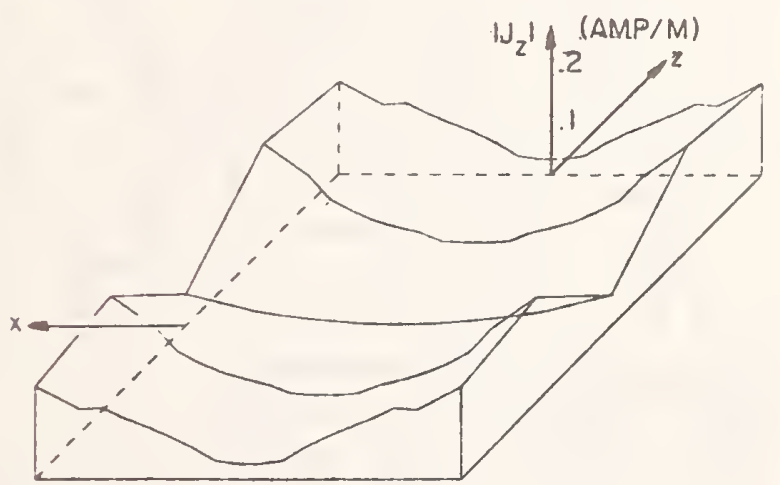

(b)

Figure 3. Normalized induced currents on Face I of Figure 2 for a $(0.5 \lambda)^{3}$ cube illuminated by a normal incidence, plane wave $\mathrm{H}_{i z}$ toward $+y$-direction (a) $\left|J_{x}\right|$, and (b) $\left|J_{z}\right| \cdot[5]$ 


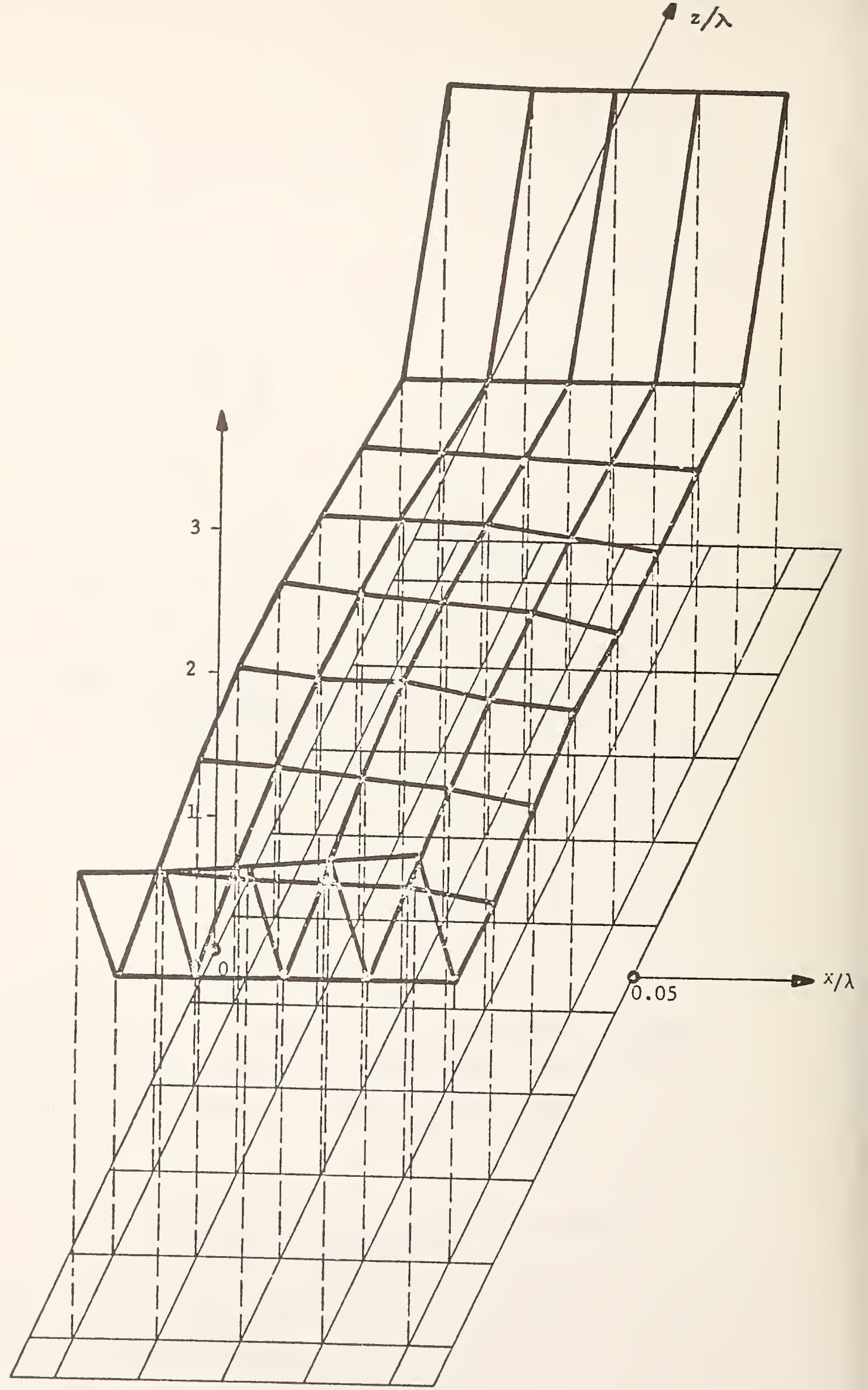

Figure 4. Normalized $\left|J_{x}\right|$ on Face I of Figure 2 for a $(0.1 \lambda)^{3^{x}}$ cube with normal incidence. 


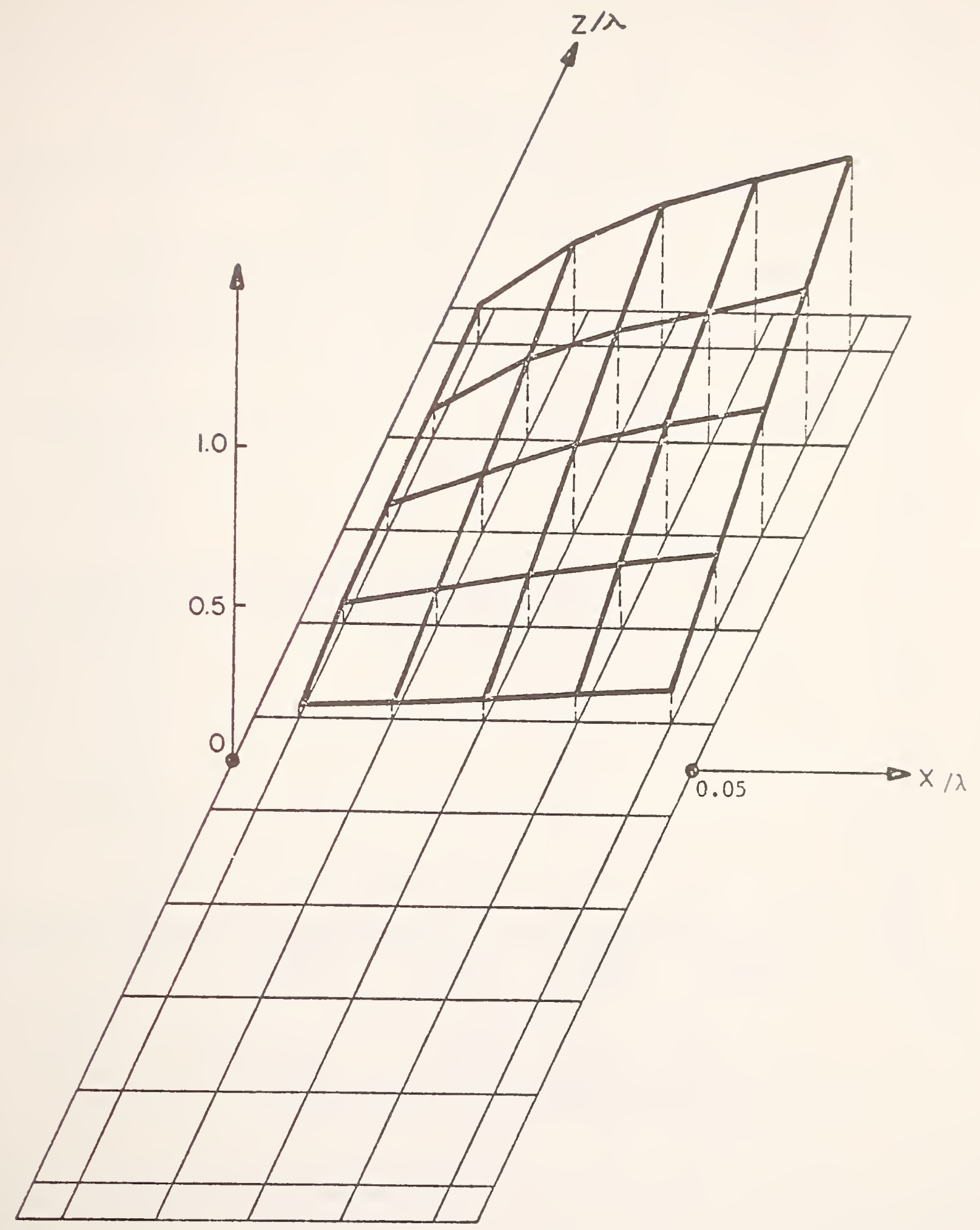

Figure 5. Normalized $\left|\mathrm{J}_{2}\right|$ on Face $I$ of Figure 2 for a $(0.1 \lambda)^{3}$ cube with normal incidence. 

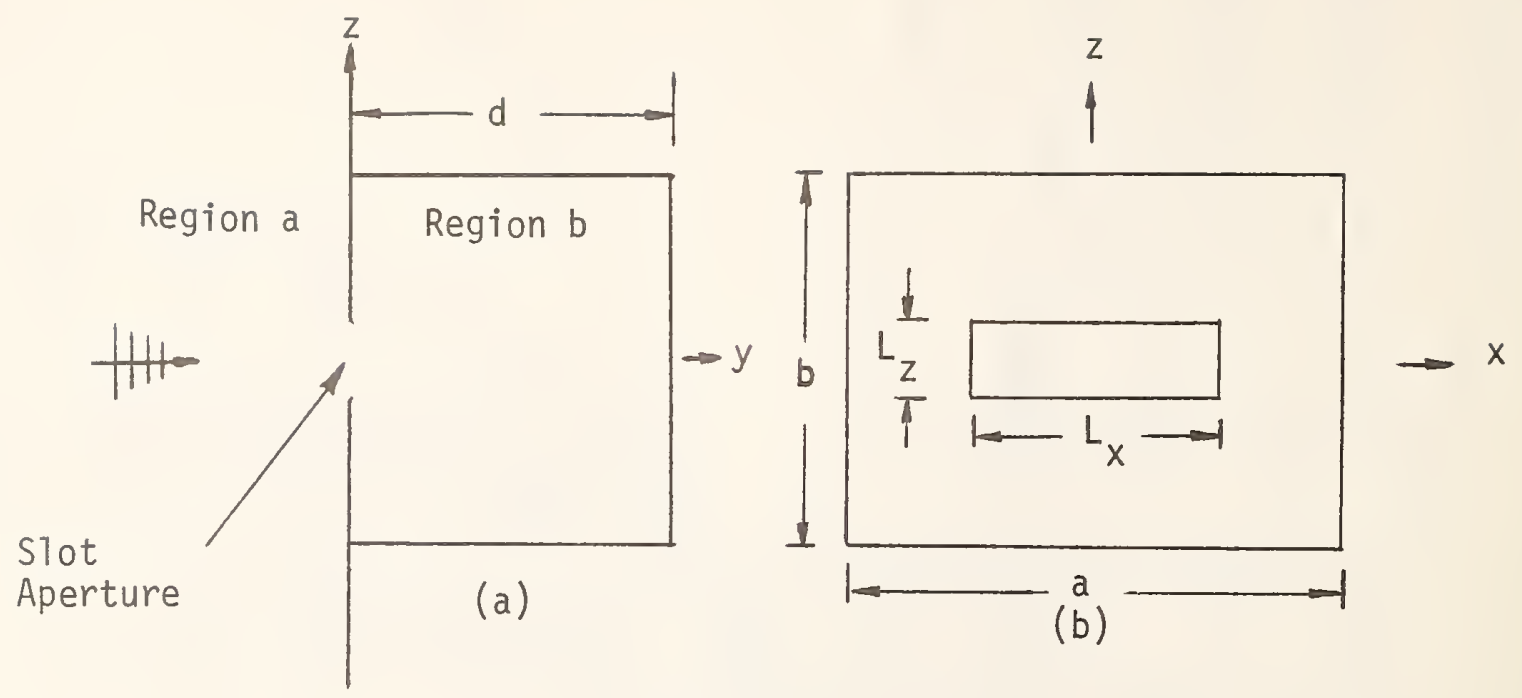

Figure 6. A plane wave incident normally on a conducting cavity with a slot aperture (a) side view, and (b) front view.
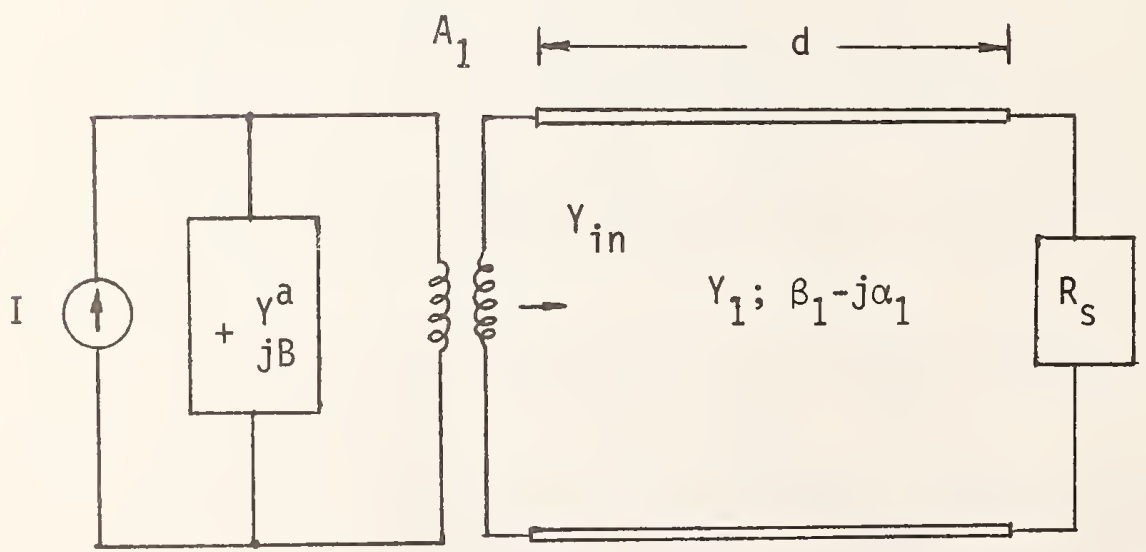

Figure 7. A network representation for the model in Figure 6. 


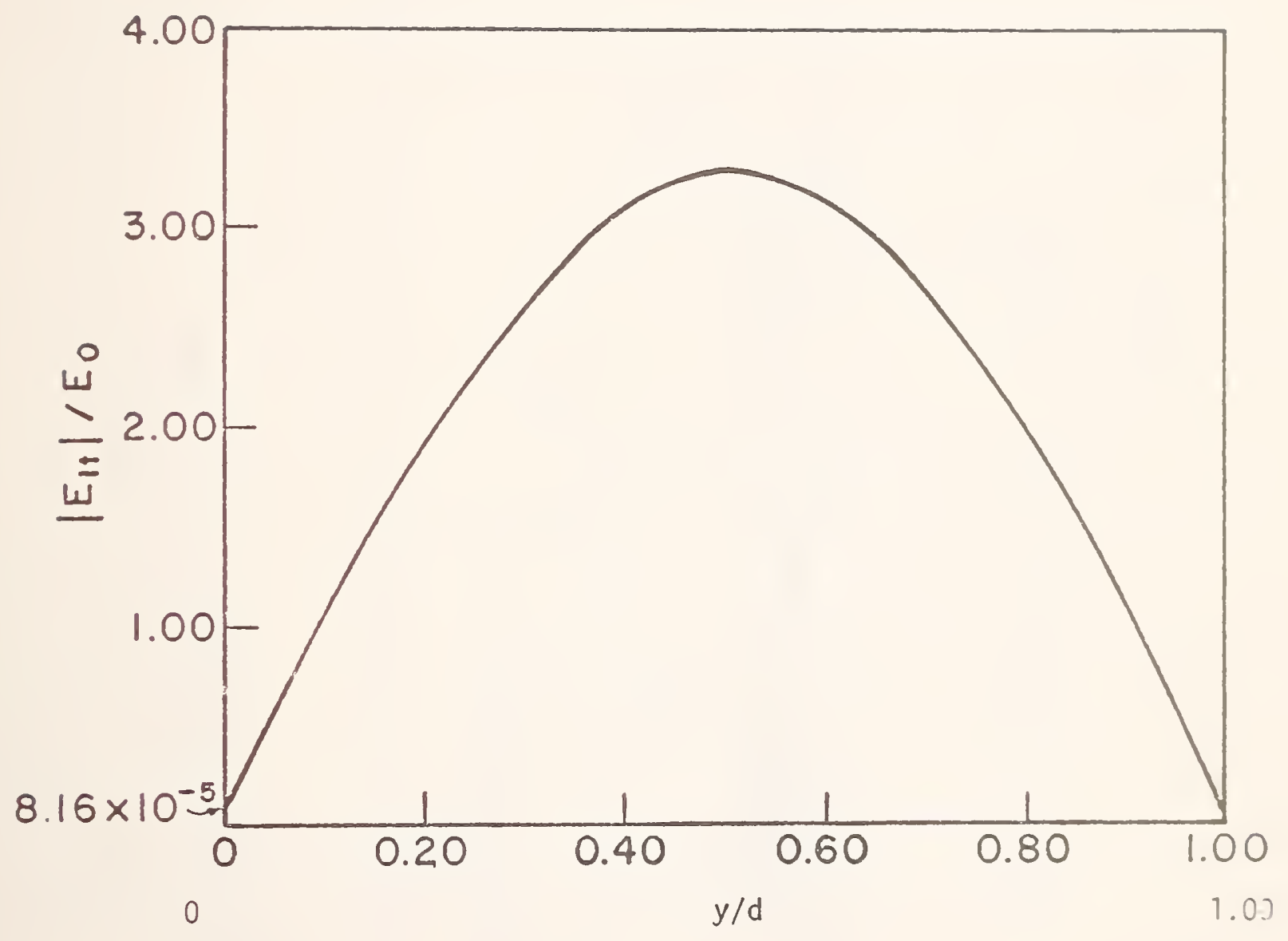

Figure 8. Electric field distribution for $T E_{10}$ mode in a copper cavity at natural resonance, $(x=z=0)$. 


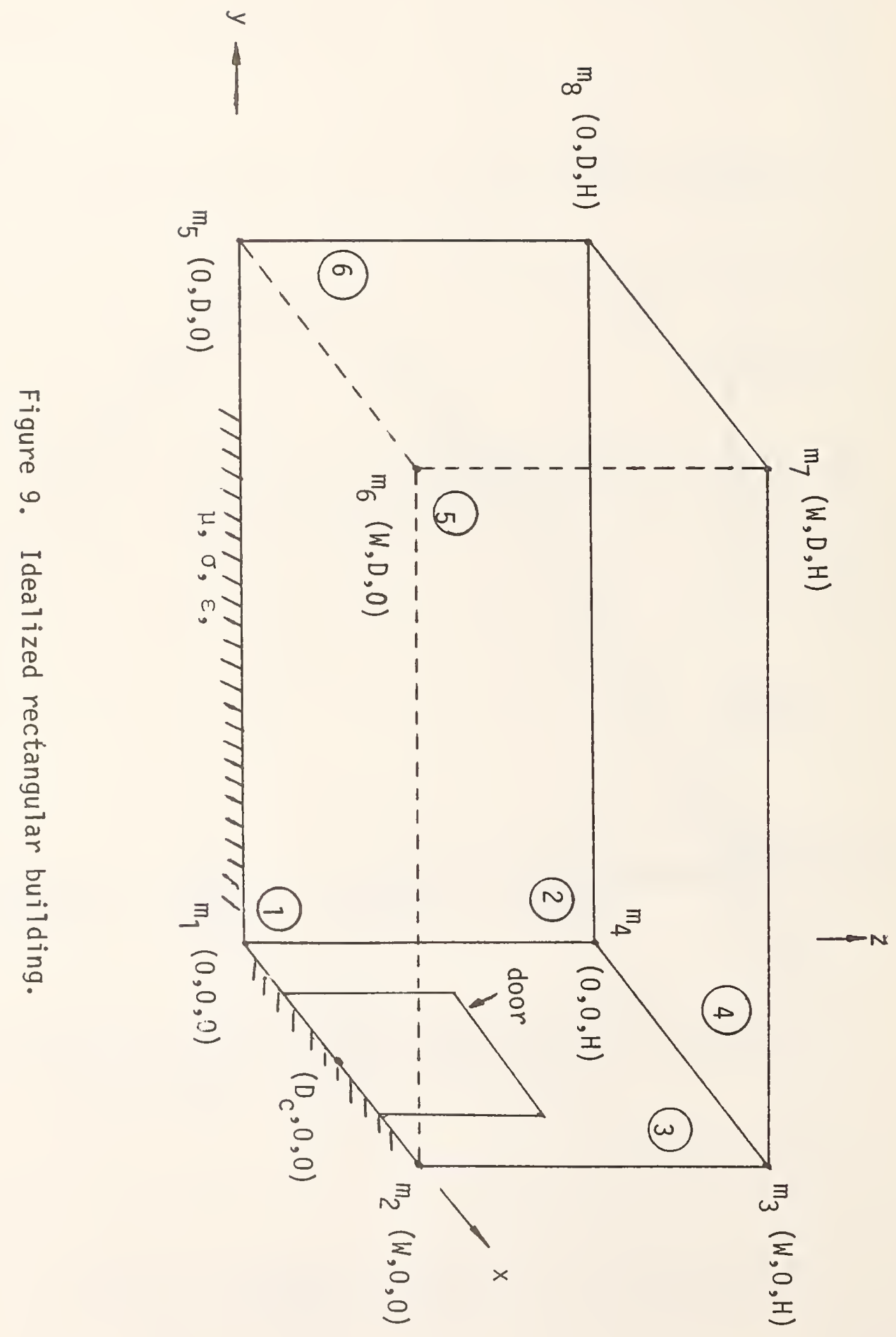




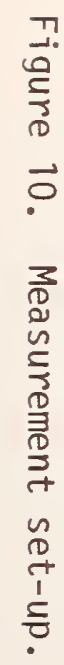

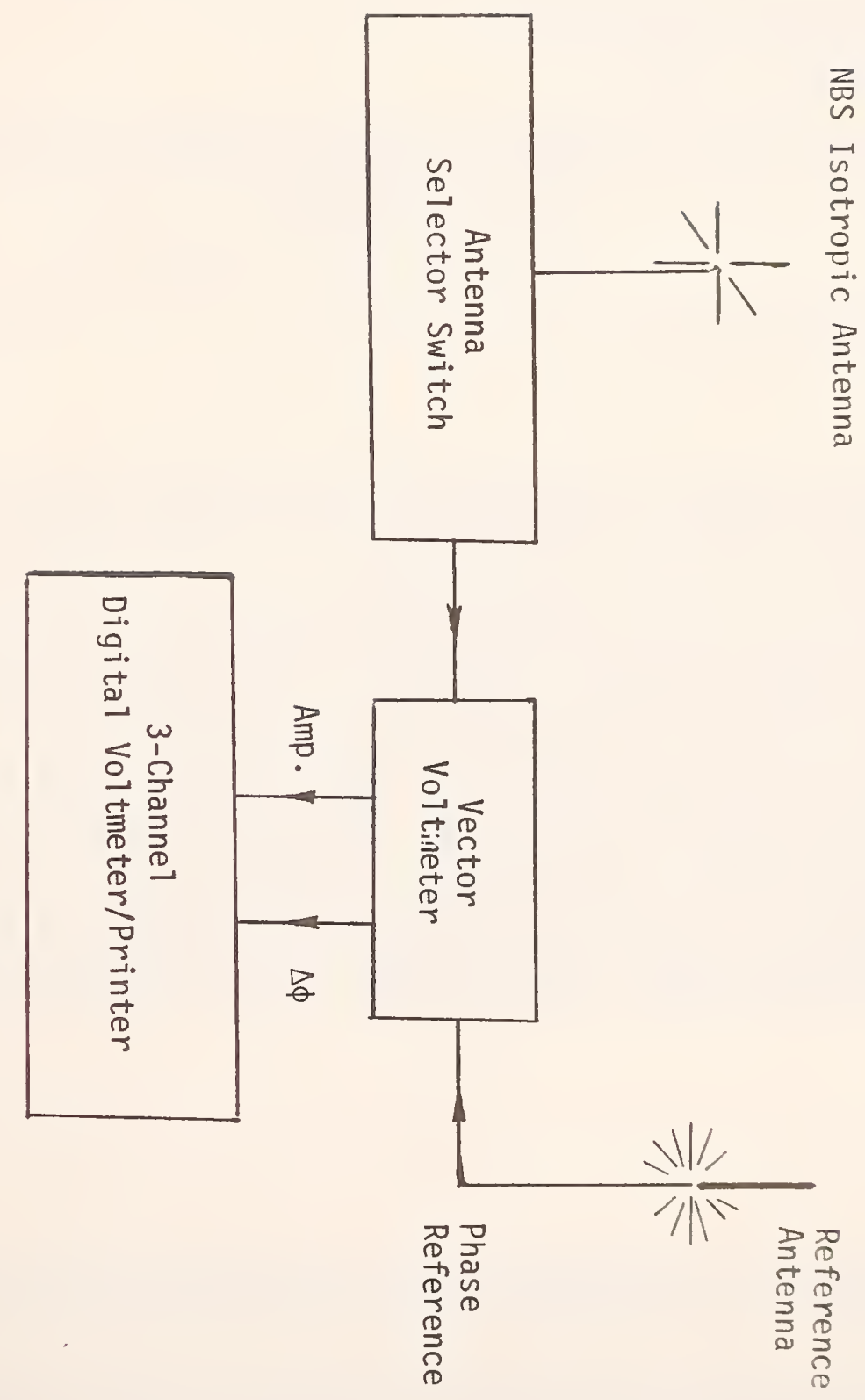




\begin{tabular}{|c|c|c|}
\hline $\begin{array}{c}\text { U.S. DEPT. OF COMM. } \\
\text { BIBLIOGRAPHIC DATA } \\
\text { SHEET (See instructions) }\end{array}$ & $\begin{array}{c}\text { 1. PUBLICATION OR } \\
\text { REPORT NO. } \\
\text { NBSIR 82-1659 }\end{array}$ & 2. Performing Organ. Report No. \\
\hline
\end{tabular}

4. TITLE AND SUBTITLE

A Study of the Electromagnetic Fields Distribution Inside Buildings with Apertures Excited by an External Source

5. $\operatorname{AUTHOR}(\mathrm{S})$

Mark T. Ma and M. G. Arthur

6. PERFORMING ORGANIZATION (If joint or other than NBS, see instructions)

NATIONAL BUREAU OF STANDARDS

DEPARTMENT OF COMMERCE

WASHINGTON, D.C. 20234

9. SPONSORING ORGANIZATION NAME AND COMPLETE ADDRESS (Street, City. State, ZIP)

Supported in part by: U.S. Army Communications-Electronics Engineering Installation Agency (USACEEIA)

Fort Huachuca, Arizona 85613

10. SUPPLEMENTARY NOTES

[] Document describes a computer program; SF-185, FIPS Software Summary, is attached.

11. ABSTRACT (A 200-word or less factual summary of most significant information. If document includes a significant bibliography or literature survey, mention it here)

Two special cases of the penetration of electromagnetic fields into a cavity, building or box are formulated and analyzed. One is to consider the case of a lossy cavity with small apertures in free space, based on an application of the equivalence principle and the use of a generalized network formulation. It is found that the field strength at the aperture center is approximately inversely proportional to the square-root of the conductivity of the cavity walls and that high field levels can exist inside the cavity under certain physical conditions. The second case is to treat the problem of large buildings with large apertures on a practical lossy ground by a combination of theoretical approach and measurement data. Field levels inside the building for this latter case depend on the transmitter power, the transmitter-to-building distance, the ground conductivity, and the measurement antenna height relative to that of the transmitter.

12. KEY WORDS (Six to twelve entries; alphabetical order; capitalize only proper names; and separate key words by semicolons)

aperture; cavity; equivalence principle; field distribution; slot.

\section{AVAILABILITY}

X] Unlimited

$\square$ For Official Distribution. Do Not Reiease to NTIS

$\square$ Order From Superintendent of Documents, U.S. Government Printing Office, Washington, D.C. 20402.

X Order From National Technical Information Service (NTIS), Springfield, VA. 22161
14. NO. OF PRINTED PAGES

123

15. Price

$\$ 12.00$ 

